\title{
GENERALIZED CLUSTER TREES AND SINGULAR MEASURES
}

\author{
By Yen-Chi Chen \\ Department of Statistics \\ University of Washington \\ JULY 12, 2018
}

\begin{abstract}
In this paper, we study the $\alpha$-cluster tree ( $\alpha$-tree) under both singular and nonsingular measures. The $\alpha$-tree uses probability contents within a set created by the ordering of points to construct a cluster tree so that it is well-defined even for singular measures. We first derive the convergence rate for a density level set around critical points, which leads to the convergence rate for estimating an $\alpha$-tree under nonsingular measures. For singular measures, we study how the kernel density estimator (KDE) behaves and prove that the KDE is not uniformly consistent but pointwise consistent after rescaling. We further prove that the estimated $\alpha$-tree fails to converge in the $L_{\infty}$ metric but is still consistent under the integrated distance. We also observe a new type of critical points-the dimensional critical points (DCPs)-of a singular measure. DCPs are points that contribute to cluster tree topology but cannot be defined using density gradient. Building on the analysis of the KDE and DCPs, we prove the topological consistency of an estimated $\alpha$-tree.
\end{abstract}

1. Introduction. Given a function $f$ defined on a smooth manifold $\mathcal{M}$, the cluster tree of $f$ is a tree structure representing the creation and merging of connected components of a level set $\left\{x: f(x) \geq f_{0}\right\}$ when we move down the level $f_{0}$ (Stuetzle, 2003; Klemelä, 2004). Because cluster trees keep track of the connected components of level sets, the shape of a cluster tree contains topological information about the underlying function $f$. Moreover, a cluster tree can be displayed on a two-dimensional plane regardless of the dimension of $\mathcal{M}$, which makes it an attractive approach for visualizing $f$. Figure 1 provides an example illustrating the construction of a cluster tree in a $1 D$ Euclidean space. In this paper, we focus on the case where $f \equiv f_{P}$ is some function of the underlying distribution $P$. In this context, the cluster tree of $f$ reveals information about $P$.

Most of cluster trees being studied in the literature are the $\lambda$-tree of a distribution (Balakrishnan et al., 2012; Chaudhuri and Dasgupta, 2010; Chaudhuri et al., 2014; Chen et al., 2016b; Kpotufe and Luxburg, 2011; Stuetzle, 2003). The $\lambda$-tree of a distribution is the cluster tree of the density function $p$ of that distribution. In this case, the tree structure contains the topological information of $p$ and we can use the $\lambda$-tree to visualize a multivariate density function. When we use an $\lambda$-tree for visualization purposes, it is also called a density tree (Klemelä, 2004, 2006, 2009).

Kent (2013) proposed a new type of cluster tree of a distribution-the $\alpha$-tree. The $\alpha$-tree uses the function $\alpha(x)=P(\{y: p(y) \leq p(x)\})$ to construct a cluster tree. Note that such a function is also called density ranking in our following paper (Chen and Dobra, 2017) and it shows great potential in analyzing GPS datasets. When the distribution is nonsingular and smooth, the $\alpha$-tree

MSC 2010 subject classifications: Primary 62G20; secondary 62G05, 62G07

Keywords and phrases: cluster tree, kernel density estimator, level set, singular measure, critical points, topological data analysis 

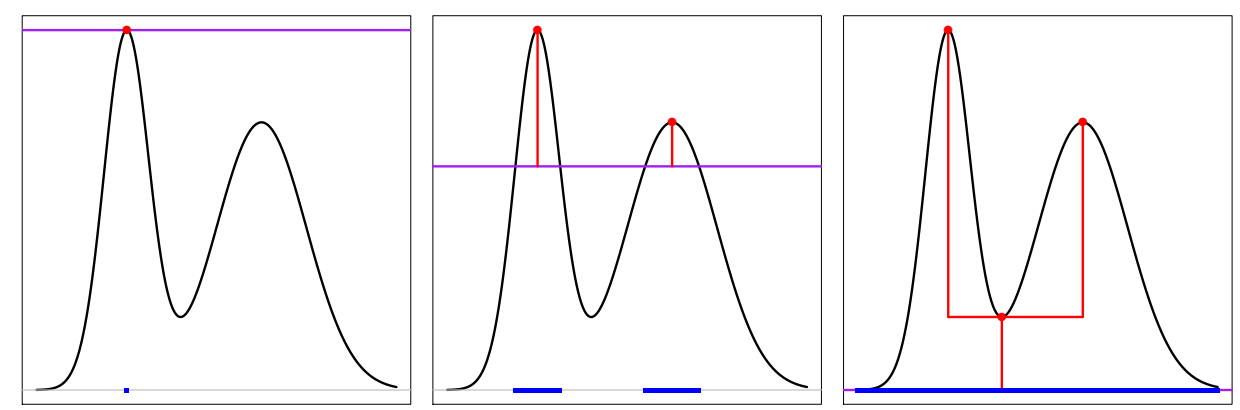

FIG 1. An example of constructing a cluster tree in $d=1$ case. The purple horizontal lines indicates the level $f_{0}$ we are using in each panel. The blue region at the bottom indicates the corresponding level set $\left\{x: f(x) \geq f_{0}\right\}$. From left to right, we gradually decrease the level $f_{0}$ and use red lines to indicate how the connected components evolve. The resulting red tree in the right panel is a cluster tree.

and the $\lambda$-tree are topologically equivalent (Lemma 1), so they both provide similar topological information for the underlying distribution. To estimate an $\alpha$-tree, we use the cluster tree of the function estimator $\widehat{\alpha}_{n}(x)=\widehat{P}_{n}\left(\left\{y: \widehat{p}_{n}(y) \leq \widehat{p}_{n}(x)\right\}\right)$ where $\widehat{P}_{n}$ is the empirical measure and $\widehat{p}_{n}$ is the kernel density estimator (KDE). Namely, we first use the KDE to estimate the density of each data point and count the number of data points with a density below the density of that given point.

When a distribution is singular, the $\lambda$-tree is ill-defined because of the lack of a probability density function, but the $\alpha$-tree is still well-defined under a mild modification. For an illustrating example, see Figure 2. These are random samples from a distribution mixed with a point mass at $x=2$ with a probability of 0.3 and a standard normal distribution with a probability of 0.7 . Thus, these samples are from a singular distribution. We generate $n=5 \times 10^{3}$ (left), $5 \times 10^{5}$ (middle), and $5 \times 10^{7}$ (right) data points and estimate the density using the KDE with the smoothing bandwidth selected by the default rule in $\mathrm{R}$. The estimated density and $\lambda$-trees (red trees) are displayed in the top row. It can be seen that when the sample size increases, $\lambda$-trees become degenerated. This is because there is no population $\lambda$-tree for this distribution. However, the $\alpha$-trees are stable in all three panels (see the bottom row of Figure 2).

Main Results. The main results of this paper are summarized as follows:

- When the distribution is nonsingular,

1. We derive the convergence rate for the estimated level set when the level equals the density value of a critical point (Theorem 3).

2. We derived the convergence rate of $\widehat{\alpha}_{n}$ (Theorem 4).

- When the distribution is singular,

3. We propose a framework that generalizes $\alpha(x)$ to define the $\alpha$-tree (Section 4 ).

4. We show that after rescaling, the KDE is pointwise, but not uniformly, consistent (Theorem 8).

5. We prove that $\widehat{\alpha}_{n}$ is inconsistent under the $L_{\infty}$ metric (Corollary 7 ) but consistent under the $L_{1}$ and $L_{1}(P)$ distance (Theorem 10).

6. We identify a new type of critical points, the dimensional critical points (DCPs), which 

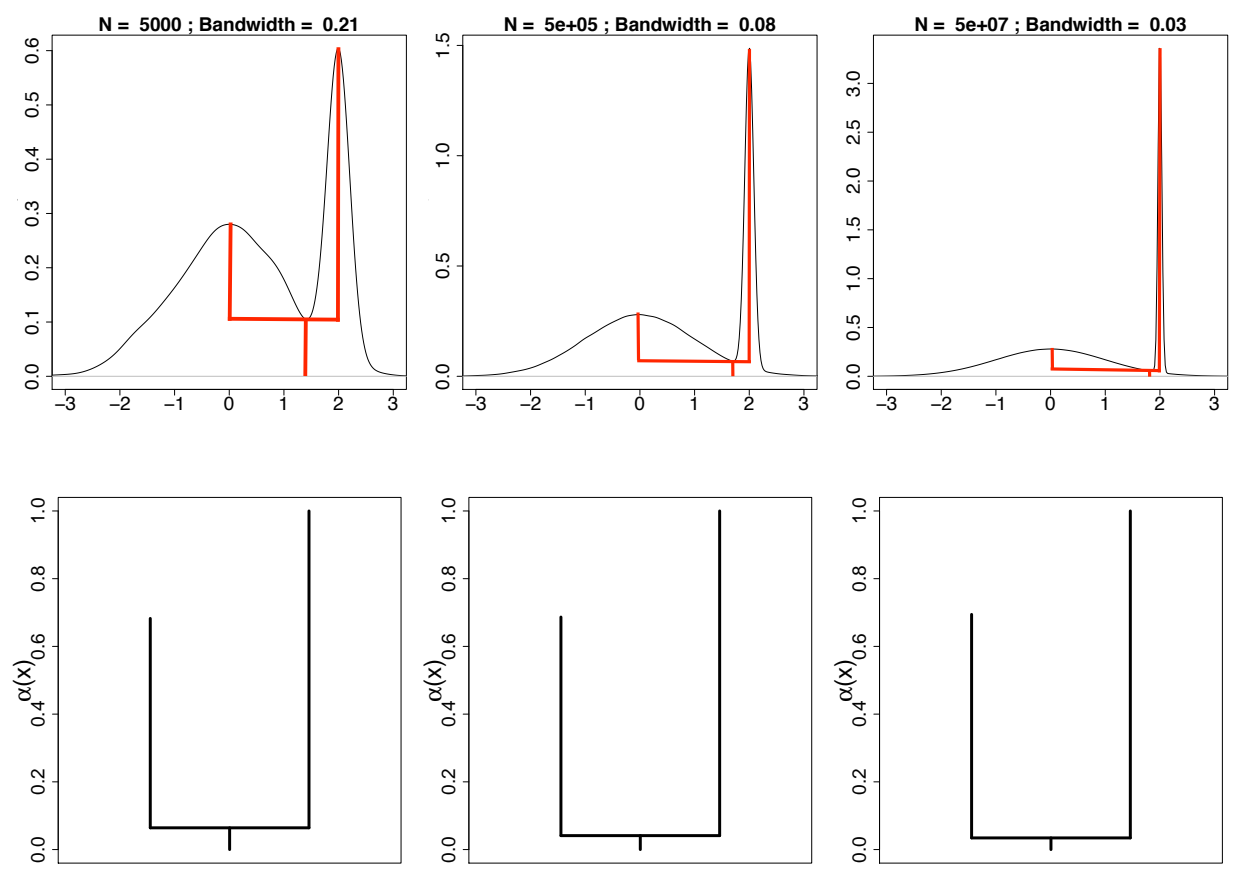

FIG 2. Example of the estimated $\lambda$-tree and $\alpha$-tree of a singular distribution. This is a random sample from a singular distribution where with a probability of 0.3 , it puts a point mass at $x=2$, and with a probability of 0.7 , we sample from a standard normal. The top panel shows the density estimated by the KDE and the red tree structure corresponds to the estimated $\lambda$-tree. The bottom panel displays the estimated $\alpha$-tree. From left to right, we increase the sample size from $5 \times 10^{3}, 5 \times 10^{5}$, to $5 \times 10^{7}$. Because the distribution is singular, there is no population $\lambda$-tree so when the smoothing bandwidth decreases (when the sample size increases), the estimated $\lambda$-tree is getting degenerated. On the other hand, the estimated $\alpha$-trees remain stable regardless of the smoothing bandwidth. Note that every level set in a $\lambda$-tree corresponds to a level set in the $\alpha$-tree but the value of the corresponding level ( $Y$-axis) will be different. The function used in constructing a $\lambda$-tree may have an unbounded range when the sample size goes to infinity, whereas the function for building an $\alpha$-tree always has a range of $[0,1]$. 
also contribute to the change in cluster tree topology. We analyze their properties in Lemma 11, 13, and 14.

7. We demonstrate that the estimated $\alpha$-tree $T_{\widehat{\alpha}_{n}}$ is topologically equivalent to the population $\alpha$-tree with probability exponentially converging to 1 (Theorem 15 ).

Related Work. There is extensive literature on theoretical aspects of the $\lambda$-tree. Notions of consistency are analyzed in Hartigan (1981); Chaudhuri and Dasgupta (2010); Chaudhuri et al. (2014); Eldridge et al. (2015). The convergence rate and the minimax theory are studied in Chaudhuri and Dasgupta (2010); Balakrishnan et al. (2012); Chaudhuri et al. (2014). Chen et al. (2016b) study how to perform statistical inference for a $\lambda$-tree. The cluster tree is also related to the topological data analysis (Carlsson, 2009; Edelsbrunner and Morozov, 2012; Wasserman, 2016). In particular, a cluster tree contains information about the zeroth order homology groups (Cohen-Steiner et al., 2007; Fasy et al., 2014; Bobrowski et al., 2017; Bubenik, 2015). In our analysis, we generalize the Morse theory to a non-smooth and even discontinuous function. Baryshnikov et al. (2013) also generalizes the Morse theory to a non-smooth function using the concept of configuration space (the collection of $n$ points in a bounded area in $R^{d}$ ). Note that their setting is different from us because we are working on a probability density function whereas the function in Baryshnikov et al. (2013) is related to pairwise distance between points and distance to the boundary of certain area. The theory of estimating a cluster tree is closely related to the theory of estimating a level set; an incomplete list of literature is as follows: Polonik (1995); Tsybakov (1997); Walther (1997); Mason and Polonik (2009); Singh et al. (2009); Rinaldo and Wasserman (2010); Steinwart (2011).

Outline. We begin with an introduction of cluster trees and the geometric concepts used in this paper in Section 2. In Section 3, we derive the convergence rate for the $\alpha$-tree estimator under nonsingular measures. In Section 4, we study the behavior of the KDE and the stability of the estimated $\alpha$-tree under singular measures. In Section 5, we investigate critical points of singular measures and derive the topological consistency of the estimated $\alpha$-tree. We summarize this paper and discuss possible future directions in Section 6. We leave all proofs in the supplementary materials (Chen, 2016).

\section{Backgrounds.}

2.1. Cluster Trees. Here we recall the definition of cluster trees in Chen et al. (2016b). Let $\mathbb{K} \subset \mathbb{R}^{d}$ and $f: \mathbb{K} \mapsto[0, \infty)$ be a function with support $\mathbb{K}$. The cluster tree of function $f$ is defined as follows.

Definition 1 (Definition 1 in Chen et al. (2016b)). For any $f: \mathbb{K} \mapsto[0, \infty)$ we define $T_{f}: \mathbb{R} \mapsto$ $2^{2^{\mathbb{K}}}$, where $2^{\mathbb{K}}$ denotes the set of all subsets of $\mathbb{K}, 2^{2^{\mathbb{K}}}$ denotes the collection of all sets of subsets of $\mathbb{K}$, and $T_{f}(\lambda)$ is the set of connected components of the upper level set $\{x \in \mathbb{K}: f(x) \geq \lambda\}$. We define the collection of connected components $\left\{T_{f}\right\}$, as $\left\{T_{f}\right\}=\bigcup_{\lambda} T_{f}(\lambda)$. Thus, $\left\{T_{f}\right\}$ is a collection of subsets of $\mathbb{K}$. We called $\left\{T_{f}\right\}$ the cluster tree of $f$.

Clearly, the cluster tree $\left\{T_{f}\right\}$ has a tree structure, because for each pair $C_{1}, C_{2} \in\left\{T_{f}\right\}$, either $C_{1} \subset C_{2}, C_{2} \subset C_{1}$, or $C_{1} \cap C_{2}=\phi$ holds.

To get a geometric understanding of the cluster tree in Definition 1, we identify edges that constitute the cluster tree. Intuitively, edges correspond to either leaves or internal branches. An 

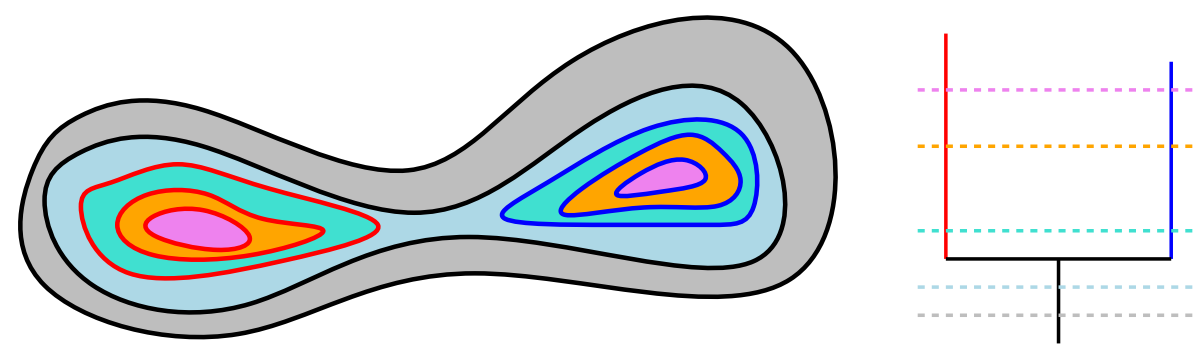

FIG 3. Connected components, edges, and edge set of a cluster tree. Left: We display connected components of level sets under five different levels (indicated by the colors: magenta, yellow, sea green, sky blue, and gray). The color of boundaries of each connected component denotes the edge they correspond to. Right: The cluster tree. We color the three edges (vertical lines) red, blue, and black. The edge set $E\left(T_{f}\right)=\left\{\mathbb{C}_{\text {red }}, \mathbb{C}_{\text {blue }}, \mathbb{C}_{\text {black }}\right\}$ and we have the ordering

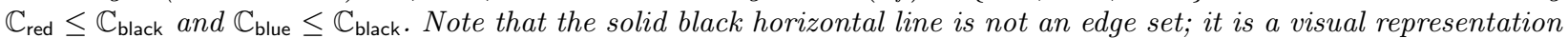
of connecting the blue and red edges to the black edge. The horizontal dashed lines indicate the five levels corresponding to the left panel. In the left panel, the three connected components with red boundaries are elements of the edge $\mathbb{C}_{\text {red. }}$

edge is roughly defined as a set of clusters whose inclusion relation with respect to clusters outside an edge is equivalent. So when the collection of connected components is divided into edges, we observe the same inclusion relation between representative clusters whenever any cluster is selected as representative for each edge.

To formally define edges, we define an interval in the cluster tree, and the equivalence relation in the cluster tree. For any two clusters $A, B \in\left\{T_{f}\right\}$, the interval $[A, B] \subset\left\{T_{f}\right\}$ is defined as a set clusters that contain $A$ and are contained in $B$, i.e.

$$
[A, B]:=\left\{C \in\left\{T_{f}\right\}: A \subset C \subset B\right\},
$$

We define the equivalence relation $\sim$ such that $A \sim B$ if and only if

$$
\begin{aligned}
\forall C \in\left\{T_{f}\right\} \text { such that } C & \notin[A, B] \cup[B, A], \\
& C \subset A \Leftrightarrow C \subset B, \quad A \subset C \Leftrightarrow B \subset C .
\end{aligned}
$$

It is easy to see that the relation $\sim$ is reflexive $(A \sim A)$, symmetric $(A \sim B$ implies $B \sim A)$, and transitive $(A \sim B$ and $B \sim C$ implies $A \sim C)$. Hence the relation $\sim$ is indeed an equivalence relation, and we can consider the set of equivalence classes $\left\{T_{f}\right\} / \sim$. We define the edge set (the collection of edges) $E\left(T_{f}\right)$ as $E\left(T_{f}\right):=\left\{T_{f}\right\} / \sim$. Each element in the edge set $\mathbb{C} \in E\left(T_{f}\right)$ is called an edge, which contains many nested connected components of the cluster tree $\left\{T_{f}\right\}$ (i.e. if $C_{1}, C_{2} \in \mathbb{C}$, then either $C_{1} \subset C_{2}$ or $C_{2} \subset C_{1}$ ). Note that every element in an edge corresponds to a connected component of an upper level set of function $f$.

To associate the edge set $E\left(T_{f}\right)$ to a tree structure, we define a partial order on the edge set as follows: let $\mathbb{C}_{1}, \mathbb{C}_{2} \in E\left(T_{f}\right)$ be two edges, we write $\mathbb{C}_{1} \leq \mathbb{C}_{2}$ if and only if $A \subset B$ for all $A \in \mathbb{C}_{1}$ and $B \in \mathbb{C}_{2}$. Then the topology of the cluster tree (the shape of the cluster tree) is completely determined by the edge set $E\left(T_{f}\right)$ and the partial order among the edge set. Figure 3 provides an example of the connected components, edges, and edge set of a cluster tree along with a tree representation.

Based on the above definitions, we define the topological equivalence between two cluster trees. 
Definition 2. For two functions $f: \mathbb{K} \mapsto[0, \infty)$ and $g: \mathbb{K} \mapsto[0, \infty)$, we say $T_{f}$ and $T_{g}$ are topological equivalent, denoted as $T_{f} \stackrel{T}{\approx} T_{g}$, if there exists a bijective mapping $S: E\left(T_{f}\right) \mapsto E\left(T_{g}\right)$ such that for any $\mathbb{C}_{1}, \mathbb{C}_{2} \in E\left(T_{f}\right)$,

$$
\mathbb{C}_{1} \leq \mathbb{C}_{2} \Longleftrightarrow S\left(\mathbb{C}_{1}\right) \leq S\left(\mathbb{C}_{2}\right)
$$

For each $\mathbb{C} \in E\left(T_{f}\right)$, we define

$$
U(\mathbb{C})=\sup \left\{\lambda: \exists C \in T_{f}(\lambda), C \in \mathbb{C}\right\}
$$

to be the maximal level of an edge $\mathbb{C}$. We define the critical tree-levels of function $f$ as

$$
\mathcal{A}_{f}=\left\{U(\mathbb{C}): \mathbb{C} \in E\left(T_{f}\right)\right\} .
$$

It is easy to see that $\mathcal{A}_{f}$ is the collection of levels of $f$ where the creation of a new connected component or the merging of two connected components occurs.

In most of the cluster tree literature (Balakrishnan et al., 2012; Chaudhuri and Dasgupta, 2010; Chaudhuri et al., 2014; Chen et al., 2016b; Eldridge et al., 2015), the cluster tree is referred to as the $\lambda$-tree, which uses the probability density function $p$ to build a cluster tree. Namely, the $\lambda$-tree is $T_{p}$.

In this paper, we focus on the $\alpha$-tree (Kent, 2013) that uses the function

$$
\alpha(x)=P(\{y: p(y) \leq p(x)\})=1-P(\{y: p(y)>p(x)\})=1-P\left(L_{p(x)}\right)
$$

to build the cluster tree $T_{\alpha}$ ( $T_{\alpha}$ is called the $\alpha$-tree). The function $\alpha(x)$ is also called density ranking in Chen and Dobra (2017). The set $L_{\lambda}=\{x: p(x) \geq \lambda\}$ is the upper level set of $p$ (note that $P(\{y: p(y)>p(x)\})=P(\{y: p(y) \geq p(x)\})$ when the density function $p$ is bounded). A feature of the $\alpha$-tree is that the function $\alpha(x)$ depends only on the ordering of points within $\mathbb{K}$. Namely, any function that assigns the same ordering of points within $\mathbb{K}$ as the density function $p$ can be used to construct the function $\alpha(x)$. Specifically, we write

$$
\begin{aligned}
& x_{1} \succ_{p} x_{2} \Leftrightarrow p\left(x_{1}\right)>p\left(x_{2}\right), \\
& x_{1} \prec_{p} x_{2} \Leftrightarrow p\left(x_{1}\right)<p\left(x_{2}\right), \\
& x_{1} \simeq_{p} x_{2} \Leftrightarrow p\left(x_{1}\right)=p\left(x_{2}\right) .
\end{aligned}
$$

Then

$$
\alpha(x)=P(\{y: p(y) \leq p(x)\})=P\left(\left\{y: y \preceq_{p} x\right\}\right),
$$

where $y \preceq_{p} x$ means either $y \prec_{p} x$ or $y \simeq_{p} x$. Note that if we replace the function $p(x)$ by $2 p(x)$ or $\log p(x)$, the ordering remains the same (i.e., $x \succ_{p} y \Leftrightarrow x \succ_{2 p} y \Leftrightarrow x \succ_{\log p} y$ ). We will use this feature later to generalize equation (2) to singular measures.

One feature of the $\alpha$-tree is that it is topological equivalent to the $\lambda$-tree. 
Lemma 1. Assume the distribution $P$ has a bounded density function $p$ and $p$ is a Morse function with a compact support. Then the $\lambda$-tree and $\alpha$-tree are topological equivalent. Namely,

$$
T_{p} \stackrel{T}{\approx} T_{\alpha}
$$

The proof of this lemma follows from the argument at the beginning of Section 4.1 of Cadre et al. (2009) so we ignore the proof. The main idea is that by equation (2) and the fact that $p$ is Morse, $\alpha$ is a strictly monotonic transformation of the density $p$ so the topology is preserved.

When we use the $\alpha$-tree, the induced upper level set

$$
\mathbb{A}_{\varpi}=\{x: \alpha(x) \geq \varpi\}
$$

is called an $\alpha$-level set.

REMARK 1 ( $\kappa$-tree). Kent (2013) also proposed another cluster tree-the $\kappa$-tree-which uses the probability content within each edge set defined by an $\alpha$-tree (or a $\lambda$-tree) to compute the function $\kappa(x)$. Because it is just a rescaling from the $\alpha$-tree, the theory of $\alpha$-tree also works for the $\kappa$-tree. For simplicity, we only study the theory of the $\alpha$-tree in this paper.

2.2. Singular Measure. When the probability measure is singular, the $\lambda$-tree is no longer welldefined because there is no density function. However, the $\alpha$-tree can still be defined.

A key feature for constructing the $\alpha$-tree is the ordering. Here we will use a generalized density function, the Hausdorff density (Preiss, 1987; Mattila, 1999), to define the $\alpha$-tree under singular measures. Given a probability measure $P$, the $s$-density ( $s$ dimensional Hausdorff density) is

$$
\mathcal{H}_{s}(x)=\lim _{r \rightarrow 0} \frac{P(B(x, r))}{C_{s} \cdot r^{s}}
$$

provided the limit exists. Note that $B(x, r)=\{y:\|y-x\| \leq r\}$ and $C_{s}$ is the $s$-dimensional volume of an $s$-dimensional unit ball for $s \geq 1$ and $C_{0}=1$.

For a given point $x$, we define the notion of generalized density using two quantities $\tau(x)$ and $\rho(x)$ :

$$
\begin{aligned}
\tau(x) & =\max \left\{s \leq d: \mathcal{H}_{s}(x)<\infty\right\} \\
\rho(x) & =\mathcal{H}_{\tau(x)}(x) .
\end{aligned}
$$

Namely, $\tau(x)$ is the 'dimension' of the probability measure at $x$ and $\rho(x)$ is the corresponding Hausdorff density at that dimension. Note that the function $\rho(x)$ is well-defined for every $x$. For any two points $x_{1}, x_{2} \in \mathbb{K}$, we define an ordering such that $x_{1} \succ_{\tau, \rho} x_{2}$ if

$$
\tau\left(x_{1}\right)<\tau\left(x_{2}\right), \quad \text { or } \quad \tau\left(x_{1}\right)=\tau\left(x_{2}\right), \quad \rho\left(x_{1}\right)>\rho\left(x_{2}\right) .
$$

That is, for any pair of points, we first compare their dimensions $\tau(x)$. The point with the lower dimensional value $\tau$ will be ranked higher than the other point. If two points have the same dimension, then we compare their corresponding Hausdorff density. When the distribution is nonsingular, 


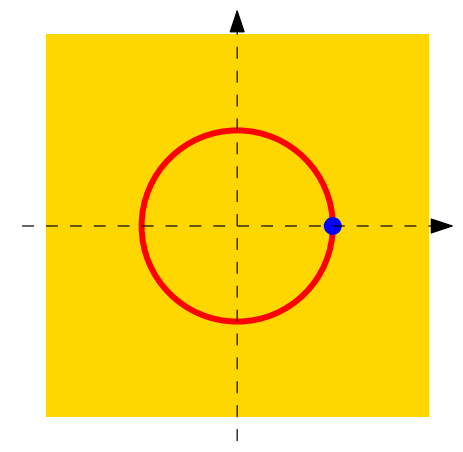

FIG 4. The supports $\mathbb{K}_{2}, \mathbb{K}_{1}$, and $\mathbb{K}_{0}$ for the bivariate random variable in Example 1 . The yellow region is $\mathbb{K}_{2}=$ $[-1,1]^{2} \backslash R_{0.5}$, where $R_{0.5}=\left\{(x, y): x^{2}+y^{2}=0.5^{2}\right\}$. The red ring is $\mathbb{K}_{1}=R_{0.5} \backslash\{(0.5,0)\}$. The blue dot is the location of $(0.5,0)$.

$\tau(x)=d$ for every $x \in \mathbb{K}$ and $\rho(x)=p(x)$ is the usual density function. Thus, the ordering is determined by the density function $p(x)$.

To define the $\alpha$-tree, we use equation (3):

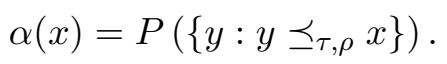

Namely, $\alpha(x)$ is the probability content of regions of points whose ordering is lower than or equal to $x$. As shown in equation (3), equation (4) is the same as equation (2) when $P$ is nonsingular. Note that by equation (4), the $\alpha$-level set $\mathbb{A}_{\varpi}=\{x: \alpha(x) \geq \varpi\}$ is well-defined in a singular measure.

2.3. Geometric Concepts. We first define some notations for sets. For a set $A$, define $\bar{A}$ to be the closure of $A, \AA$ to be the interior of $A, \partial A$ to be the boundary of set $A$, and $A^{C}=\mathbb{K} \backslash A$ to be the complement of set $A$ restricted to the support $\mathbb{K}$. When $A$ is a manifold, $\partial A$ and $\AA$ will be the boundary and interior of the manifold respectively. We define $A \triangle B=(A \backslash B) \cup(B \backslash A)$ to be the symmetric difference for sets $A$ and $B$

Based on the definition of $\tau(x)$, we decompose the support $\mathbb{K}$ into

$$
\mathbb{K}=\mathbb{K}_{d} \bigcup \mathbb{K}_{d-1} \bigcup \cdots \bigcup \mathbb{K}_{0},
$$

where $\mathbb{K}_{s}=\{x: \tau(x)=s\}$. Thus, $\left\{\mathbb{K}_{0}, \cdots, \mathbb{K}_{d}\right\}$ forms a partition of the entire support $\mathbb{K}$. We call each $\mathbb{K}_{s}$ an $s$-dimensional support. When we analyze the support $\mathbb{K}_{s}$, any $\mathbb{K}_{s^{\prime}}$ with $s^{\prime}>s$ is called a higher dimensional support (with respect to $\mathbb{K}_{s}$ ) and $s^{\prime}<s$ will be called a lower dimensional support.

EXAMPLE 1. Let $X$ be a bivariate random variable such that (i) with a probability of 0.7, it is from a uniform distribution on $[-1,1]^{2}$, (ii) with a probability of 0.25 , it is from a uniform distribution on the ring $R_{0.5}=\left\{(x, y): x^{2}+y^{2}=0.5^{2}\right\}$, (iii) with a probability of 0.05 , it is equal to $(0.5,0)$. Apparently, the distribution of $X$ is singular and the support $\mathbb{K}=[-1,1]^{2}$. In this case, $\mathbb{K}_{2}=[-1,1]^{2} \backslash R_{0.5}, \mathbb{K}_{1}=R_{0.5} \backslash\{(0.5,0)\}$, and $\mathbb{K}_{0}=\{(0.5,0)\}$. Figure 4 shows these supports under different colors. The yellow rectangular region is $\mathbb{K}_{2}$, the red ring area is $\mathbb{K}_{1}$, and the blue dot denotes the location of $\mathbb{K}_{0}$. 
To regularize the behavior of $\rho(x)$ on each support $\mathbb{K}_{s}$, we assume that the closure of the support, $\overline{\mathbb{K}_{s}}$, is an $s$-dimensional smooth manifold (properties of a smooth manifold can be found in Lee 2012; Tu 2010).

For an $s$-dimensional smooth manifold $\mathcal{M}$, the tangent space on each point of $\mathcal{M}$ changes smoothly ( $\mathrm{Tu}, 2010 ;$ Lee, 2012). Namely, for $x \in \mathcal{M}$, we can find an orthonormal basis $\left\{v_{1}(x), \cdots, v_{s}(x)\right.$ : $\left.v_{\ell}(x) \in \mathbb{R}^{d}, \ell=1, \cdots, s\right\}$ such that $\left\{v_{1}(x), \cdots, v_{s}(x)\right\}$ spans the tangent space of $\mathcal{M}$ at $x$ and each $v_{\ell}(x)$ is a smooth (multivalued) function on $\mathcal{M}_{s}$. For simplicity, for $x \in \mathbb{K}_{s}$, we denote $T_{s}(x)$ as the tangent space of $\mathbb{K}_{s}$ at $x, N_{s}(x)$ as the normal space of $\mathbb{K}_{s}$ at $x$, and $\nabla_{T_{s}(x)}$ to be taking the derivative in the tangent space.

For a function $f: \mathcal{M} \mapsto \mathbb{R}$ defined on a smooth manifold $\mathcal{M}$, the function $f$ is a Morse function (Milnor, 1963; Morse, 1925, 1930) if all its critical points are non-degenerate. Namely, the eigenvalues of the Hessian matrix of $f$ at each critical point are non-zero. When the function $f$ is a Morse function, its $\lambda$-tree is stable (Chazal et al., 2014; Chen et al., 2016b) in the $L_{\infty}$ metric under a small perturbation of $f$.

To link the concept of the Morse function to the Hausdorff density $\rho(x)$, we introduce a generalized density

$$
\rho_{s}^{\dagger}: \overline{\mathbb{K}_{s}} \mapsto[0, \infty)
$$

such that $\rho_{s}^{\dagger}(x)=\lim _{x_{n} \in \mathbb{K}_{s}: x_{n} \rightarrow x} \rho\left(x_{n}\right)$ provided the limit exists and does not depend on the choice of the sequence $x_{n}$. It is easy to see that $\rho_{s}^{\dagger}(x)=\rho(x)$ when $x \in \mathbb{K}_{s}$ but now it is defined on a smooth manifold $\overline{\mathbb{K}}_{s}$. We say $\rho(x)$ is a generalized Morse function if the corresponding $\rho_{s}^{\dagger}(x)$ is a Morse function for $s=1, \cdots, d$. Later we will show that this generalization leads to a stable $\alpha$-tree for a singular measure.

For $\overline{\mathbb{K}}_{s}$, let $\mathcal{C}_{s}=\left\{x \in \overline{\mathbb{K}}_{s}: \nabla_{T_{s}(x)} \rho_{s}^{\dagger}(x)=0\right\}$ be the collection of its critical points. Then the fact that $\rho_{s}^{\dagger}(x)$ is a Morse function implies that the eigenvalues of the Hessian matrix $\nabla_{T_{s}(c)} \nabla_{T_{s}(c)} \rho_{s}^{\dagger}(c)$ are non-zero for every $c \in \mathcal{C}_{s}$. We call $g_{s}(x)=\nabla_{T_{s}(x)} \rho_{s}^{\dagger}(x)$ the generalized gradient and $H_{s}(x)=$ $\nabla_{T_{s}(x)} \nabla_{T_{s}(x)} \rho_{s}^{\dagger}(x)$ the generalized Hessian. For the case $s=0$ (point mass), we define $\mathcal{C}_{0}=\mathbb{K}_{0}$. The collection $\mathcal{C}=\bigcup_{s=1, \cdots, d} \mathcal{C}_{s}$ is called the collection of generalized critical points of $\rho(x)$. Each element $c \in \mathcal{C}$ is called a generalized critical point.

Finally, we introduce the concept of reach (Federer, 1959; Chen et al., 2015a) for a smooth manifold $\mathcal{M}$. The reach of $\mathcal{M}$ is defined as

$$
\operatorname{reach}(\mathcal{M})=\sup \{r \geq 0 \text { : every point in } \mathcal{M} \oplus r \text { has a unique projection onto } \mathcal{M}\} \text {, }
$$

where $A \oplus r=\{x: d(x, A) \leq r\}$. One can view reach as the radius of the largest ball that can roll freely outside $\mathcal{M}$. More details about reach can be found in Federer (1959); Chen et al. (2015a). Reach plays a key role in the stability of a level set; see Chen et al. (2015a) for more details.

REMARK 2. If we further assume that the supports satisfy

$$
\mathbb{K} \supset \overline{\mathbb{K}}_{d} \supset \overline{\mathbb{K}}_{d-1} \supset \cdots \supset \overline{\mathbb{K}}_{0},
$$

then the support $\mathbb{K}$ forms a stratified space (Goresky and MacPherson, 1980, 2012). Roughly speaking, a stratified space is a topological space $\mathbb{W}$ such that there exists a decomposition (called stratification) $\mathbb{W}_{0}, \cdots, \mathbb{W}_{d}$ of $\mathbb{W}$ with the properties that (i) each $\mathbb{W}_{k}$ is a $k$-dimensional smooth manifold, (ii) $\mathbb{W}=\bigcup_{\omega=0}^{d} \mathbb{W}_{\omega}$, and (iii) for any $k \leq \ell$,

$$
\mathbb{W}_{k} \cap \overline{\mathbb{W}}_{\ell} \neq \emptyset \Leftrightarrow \mathbb{W}_{k} \subset \overline{\mathbb{W}}_{\ell}
$$


From the properties of a stratified space, one can see how equation (6) is related to a stratified space. Note that for a stratified space, if we consider a probability measure that is a mixture of probability measures defined on each stratum $\left(\mathbb{W}_{k}\right)$, this defines a singular measure as the one being considered in this paper. The topology of a stratified space can be defined using the intersection homology (Goresky and MacPherson, 1980; Edelsbrunner and Harer, 2008; Friedman, 2014). The notion of intersection homology and stratified space will be particularly useful if we want to work on higher-order homology groups.

2.4. Estimating the $\alpha$ function and the $\alpha$-tree. In this paper, we focus on estimating $\alpha$-trees via the KDE:

$$
\widehat{p}_{n}(x)=\frac{1}{n h^{d}} \sum_{i=1}^{n} K\left(\frac{\left\|x-X_{i}\right\|}{h}\right) .
$$

Specifically, we first estimate the density by $\widehat{p}_{n}$ and then construct the estimator $\widehat{\alpha}_{n}$ :

$$
\widehat{\alpha}_{n}(x)=\widehat{P}_{n}\left(\left\{y: \widehat{p}_{n}(y) \leq \widehat{p}_{n}(x)\right\}\right)
$$

where $\widehat{P}_{n}$ is the empirical measure and $\widehat{L}_{\lambda}=\left\{x: \widehat{p}_{n}(x) \geq \lambda\right\}$. Note that when $x$ does not contain any point mass of $P, \widehat{\alpha}_{n}(x)=1-\widehat{P}_{n}\left(\widehat{L}_{\widehat{p}_{n}(x)}\right)$.

To quantify the uncertainty in the estimator $\widehat{\alpha}_{n}$, we consider three error measurements. The first error measurement is the $L_{\infty}$ error, which is defined as

$$
\left\|\widehat{\alpha}_{n}-\alpha\right\|_{\infty}=\sup _{x}\left|\widehat{\alpha}_{n}(x)-\alpha(x)\right| .
$$

The $L_{\infty}$ error has been used in several cluster tree literature; see, e.g., Eldridge et al. (2015); Chen et al. (2016b). An appealing feature of the $L_{\infty}$ error is that this quantity is the same (up to some constant) as some other tree error metrics such as the merge distortion metric (Eldridge et al., 2015). Convergence in the merge distortion metric implies the Hartigan consistency (Eldridge et al., 2015), a notion of consistency of a cluster tree estimator described in Hartigan (1981); Chaudhuri and Dasgupta (2010); Chaudhuri et al. (2014). Thus, because of the equivalence between the $L_{\infty}$ error and the merge distortion metric, convergence in $L_{\infty}$ implies the Hartigan consistency of an estimated cluster tree.

The other two errors are the integrated error and the probability error (probability-weighted integrated error). Both are common error measurements for evaluating the quality of a function estimator (Wasserman, 2006; Scott, 2015). The integrated error is

$$
\left\|\widehat{\alpha}_{n}-\alpha\right\|_{\mu}=\int\left|\widehat{\alpha}_{n}(x)-\alpha(x)\right| d x
$$

which is also known as the integrated distance or $L_{1}$ distance. The probability error (probabilityweighted integrated error) is

$$
\left\|\widehat{\alpha}_{n}-\alpha\right\|_{P}=\int\left|\widehat{\alpha}_{n}(x)-\alpha(x)\right| d P(x),
$$

which is the integrated distance weighted by the probability measure, which is also known as $L_{1}(P)$ distance. The integrated error and the probability error are more robust than the $L_{\infty}$ error-a large difference in a small region will not affect on these errors much. 
To quantify the uncertainty in the topology of $\alpha$-tree, we introduce the notion of topological error, which is defined as

$$
P\left(T_{\widehat{\alpha}_{n}} \stackrel{T}{\approx} T_{\alpha}\right)=1-P\left(T_{\widehat{\alpha}_{n}} \stackrel{T}{\approx} T_{\alpha}\right) .
$$

Namely, the topological error is the probability that the estimated $\alpha$-tree is not topological equivalent to the population $\alpha$-tree.

Finally, we define the following notations. For a smooth function $p$, we define $\|p\|_{\ell, \infty}$ as the supremum maximal norm of $\ell$-th derivative of $p$. For instance,

$$
\|p\|_{0, \infty}=\sup _{x \in \mathbb{K}} p(x), \quad\|p\|_{1, \infty}=\sup _{x \in \mathbb{K}}\|g(x)\|_{\max }, \quad\|p\|_{2, \infty}=\sup _{x \in \mathbb{K}}\|H(x)\|_{\max },
$$

where $g(x)=\nabla p(x)$ and $H(x)=\nabla \nabla p(x)$ are the gradient and Hessian matrix of $p(x)$, respectively. A vector $\beta=\left(\beta_{1}, \ldots, \beta_{d}\right)$ of non-negative integers is called a multi-index with $|\beta|=\beta_{1}+\beta_{2}+\cdots+\beta_{d}$ and the corresponding derivative operator is

$$
D^{\beta}=\frac{\partial^{\beta_{1}}}{\partial x_{1}^{\beta_{1}}} \cdots \frac{\partial^{\beta_{d}}}{\partial x_{d}^{\beta_{d}}},
$$

where $D^{\beta} f$ is often written as $f^{(\beta)}$.

3. Theory for Nonsingular Measures. To study the theory for nonsingular measures, we make the following assumptions.

\section{Assumptions.}

(P1) $p$ has a compact support $\mathbb{K}$ and is a Morse function and is four times differentiable with $\|p\|_{\ell, \infty}<\infty$ for $\ell=0, \cdots, 4$.

(K1) $K(x)$ has compact support and is non-increasing on $[0,1]$, and has at least fourth-order bounded derivative and

$$
\int\|x\|^{2} K^{(\beta)}(\|x\|) d x<\infty, \quad \int\left(K^{(\beta)}(\|x\|)\right)^{2} d x<\infty
$$

(K2) Let

for $|\beta| \leq 2$ and $K^{(2)}(0)<0$.

$$
\mathcal{K}_{r}=\left\{y \mapsto K^{(\beta)}\left(\frac{\|x-y\|}{h}\right): x \in \mathbb{R}^{d},|\beta|=r, \bar{h}>h>0\right\},
$$

where $K^{(\beta)}$ is defined in equation (8) and $\mathcal{K}_{l}^{*}=\bigcup_{r=0}^{l} \mathcal{K}_{r}$ and $\bar{h}$ is some positive number. We assume that $\mathcal{K}_{2}^{*}$ is a VC-type class. i.e. there exists constants $A, v$ and a constant envelope $b_{0}$ such that

$$
\sup _{Q} N\left(\mathcal{K}_{2}^{*}, \mathcal{L}^{2}(Q), b_{0} \epsilon\right) \leq\left(\frac{A}{\epsilon}\right)^{v}
$$

where $N\left(T, d_{T}, \epsilon\right)$ is the $\epsilon$-covering number for a semi-metric set $T$ with metric $d_{T}$ and $\mathcal{L}^{2}(Q)$ is the $L_{2}$ norm with respect to the probability measure $Q$. 
Assumption (P1) is a common condition to guarantee that critical points are well-separated and will not move too far away under a small perturbation on the gradient and Hessian of the density function (Chazal et al., 2014; Chen et al., 2016b). We need the fourth-order derivative to ensure the estimated density Hessian matrix converges to the population density Hessian matrix (the bias in estimating the Hessian matrix depends on fourth-order derivatives). Assumption (K1) is a standard condition on kernel function (Wasserman, 2006; Scott, 2015). Assumption (K2) regularizes the complexity of kernel functions so we have uniform bounds on density, gradient, and Hessian estimation. It was first proposed by Giné and Guillou (2002) and Einmahl and Mason (2005) and was later used in various studies such as Genovese et al. (2009, 2014); Chen et al. (2015b).

We first study the error rates under nonsingular measures. In the case of the $\lambda$-tree, error rates are well-studied, and we summarize them in the following theorem.

Theorem 2. Assume (P1, K1-2). Then when $h \rightarrow 0, \frac{n h^{d+4}}{\log n} \rightarrow \infty$,

$$
\begin{array}{r}
\left\|\widehat{p}_{n}-p\right\|_{\infty}=O\left(h^{2}\right)+O_{P}\left(\sqrt{\frac{\log n}{n h^{d}}}\right) \\
\left\|\widehat{p}_{n}-p\right\|_{\mu}=O\left(h^{2}\right)+O_{P}\left(\sqrt{\frac{1}{n h^{d}}}\right) \\
\left\|\widehat{p}_{n}-p\right\|_{P}=O\left(h^{2}\right)+O_{P}\left(\sqrt{\frac{1}{n h^{d}}}\right) \\
P\left(T_{\widehat{p}_{n}} \stackrel{T}{\approx} T_{p}\right) \geq 1-c_{0} \cdot e^{-c_{1} \cdot n h^{d+4}},
\end{array}
$$

for some $c_{0}, c_{1}>0$.

The rate of consistency under the $L_{\infty}$ error can be found in Chen et al. (2015a); Giné and Guillou (2002); Einmahl and Mason (2005). The integrated error and probability error can be seen in Scott (2015). And the topological error bound follows Lemma 2 in Chen et al. (2016b) and the concentration of $L_{\infty}$ metric for the estimated Hessian matrix.

The requirement of $h$ in Theorem 2 enforces the uniform convergence of the KDE as well as its first and second derivative. Uniform convergence of derivatives of the KDE implies the convergence of some geometric structures of the density function, such as the ridges (Chen et al., 2015b; Genovese et al., 2014), critical points (Chazal et al., 2014; Chen et al., 2016a), and persistent diagrams (Cohen-Steiner et al., 2007; Fasy et al., 2014; Chen, 2017).

Now we turn to the consistency for $\alpha$-tree. To derive the rate for the $\alpha$-tree, we need to study the convergence rate of an estimated level set when the level is the density value of a critical point (also known as a critical level). The reason is that the quantity $\alpha(x)=1-P\left(L_{p(x)}\right)$ is the probability content of upper level set $L_{p(x)}=\{y: p(y) \geq p(x)\}$. When $p(x)=p(c)$ for some critical point $c$ of $p$, we face the problem of analyzing the stability of level sets at a critical level.

Theorem 3 (Level set error at a critical value). Assume (P1) and (K1-2) and $d \geq 2$. Let $\lambda$ be a density level corresponding to the density of a critical point. When $h \rightarrow 0, \frac{\log n}{n h^{d+4}} \rightarrow 0$,

$$
\mu\left(\widehat{L}_{\lambda} \triangle L_{\lambda}\right)=O_{P}\left(\left\|\widehat{p}_{n}-p\right\|_{\infty}^{\frac{d}{d+1}}\right) .
$$


The rate in Theorem 3 is slower than the usual density estimation rate. This is because the boundary of $L_{\lambda}$ hits a critical point when $\lambda$ equals the density of a critical point. The regions around a critical point have a very low gradient, which leads to a slower convergence rate. It is well-known (Giné and Guillou, 2002; Einmahl and Mason, 2005; Genovese et al., 2014) that under assumption $(\mathrm{P})$ and $(\mathrm{K} 1-2)$,

$$
\left\|\widehat{p}_{n}-p\right\|_{\infty}=O\left(h^{2}\right)+O_{P}\left(\sqrt{\frac{\log n}{n h^{d}}}\right) .
$$

In Theorem 3, we see that when $d$ is large, the quantity $\frac{d}{d+1} \rightarrow 1$ so the error rate is similar to $\left\|\widehat{p}_{n}-p\right\|_{\infty}$. This is because the regions that slow down the error rate are areas around the critical points. These areas occupy a small volume when $d$ is large, which decreases the difference in the rate.

REMARK 3. Theorem 3 complements many existing level set estimation theories. To our knowledge, no literature has worked on the situation where $\lambda$ equals the density of a critical point. Level set theories mostly focus on one of the following three cases: (i) the gradient on the level set boundary $\partial L_{\lambda}=\{x: p(x)=\lambda\}$ is bounded away from 0 (Molchanov, 1991; Tsybakov, 1997; Walther, 1997; Cadre, 2006; Laloe and Servien, 2013; Mammen and Polonik, 2013; Chen et al., 2015a), (ii) a lower bound on the density changing rate around level $\lambda$ (Singh et al., 2009; Rinaldo et al., 2012), (iii) an $(\epsilon, \sigma)$ condition for density (Chaudhuri and Dasgupta, 2010; Chaudhuri et al., 2014). When $\lambda$ equals a critical level, none of these assumptions hold.

Based on Theorem 3, we derive the convergence rate of $\widehat{\alpha}_{n}$.

TheOrem 4. Assume (P1) and (K1-2) and $d \geq 2$ and the smoothing bandwidth satisfies $h \rightarrow$ $0, \frac{\log n}{n h^{d+4}} \rightarrow 0$. Let $\mathcal{C}=\{x: \nabla p(x)=0\}$ be the collection of critical points and let $a_{n}$ be a sequence of $n$ such that $\left\|\widehat{p}_{n}-p\right\|_{\infty}=o\left(a_{n}\right)$. Then uniformly for all $x$,

$$
\widehat{\alpha}_{n}(x)-\alpha(x)= \begin{cases}O_{P}\left(\left\|\widehat{p}_{n}-p\right\|_{\infty}\right) & , \text { if }|p(x)-p(c)|>a_{n} \text { for all } c \in \mathcal{C}, \\ O_{P}\left(\left\|\widehat{p}_{n}-p\right\|_{\infty}^{\frac{d}{d+1}}\right) & , \text { otherwise }\end{cases}
$$

Theorem 4 shows uniform error rates for $\widehat{\alpha}_{n}$. When the density of a given point is away from critical levels, the rate follows the usual density estimation rate. When the given point has a density value close to some critical points, the rate is slowed down by the low gradient areas around critical points. Note that the sequence $a_{n}$ is to make the bound uniform for all $x$. To obtain an integrated error rate (and the probability error rate) of $\widehat{\alpha}_{n}$, we can choose $a_{n}=\frac{1}{\log n}\left(O\left(h^{2}\right)+O_{P}\left(\sqrt{\frac{1}{n h^{d}}}\right)\right)$ which leads to the following result. 
Corollary 5. Assume (P1) and (K1-2) and $d \geq 2$. Then when $h \rightarrow 0, \frac{n h^{d+4}}{\log n} \rightarrow \infty$,

$$
\begin{gathered}
\left\|\widehat{\alpha}_{n}-\alpha\right\|_{\infty}=O\left(h^{\frac{2 d}{d+1}}\right)+O_{P}\left(\left(\frac{\log n}{n h^{d}}\right)^{\frac{d}{2(d+1)}}\right) \\
\left\|\widehat{\alpha}_{n}-\alpha\right\|_{\mu}=O\left(h^{2}\right)+O_{P}\left(\sqrt{\frac{\log n}{n h^{d}}}\right) \\
\left\|\widehat{\alpha}_{n}-\alpha\right\|_{P}=O\left(h^{2}\right)+O_{P}\left(\sqrt{\frac{\log n}{n h^{d}}}\right) \\
P\left(T_{\widehat{\alpha}_{n}} \stackrel{T}{\approx} T_{\alpha}\right) \geq 1-c_{0} \cdot e^{-c_{1} \cdot n h^{d+4}},
\end{gathered}
$$

for some $c_{0}, c_{1}>0$.

Comparing Corollary 5 to Theorem 2, we see that only the $L_{\infty}$ error rate has a major difference and the other two errors differ by a $\sqrt{\log n}$ factor. This is because Theorem 4 proves that, only at the level of a critical point, we will have a slower convergence rate. Thus, the $L_{\infty}$ error will be slowed down by these points. However, the collection of points $\{x: p(x)=p(c)$ for some $c \in \mathcal{C}\}$ has Lebesgue measure zero so the slow convergence rate does not translate to the integrated error and the probability error. The topological error follows from Theorem 2 and Lemma $1: T\left(\widehat{p}_{n}\right) \stackrel{T}{\approx}$ $T_{\widehat{\alpha}_{n}}, T(p) \stackrel{T}{\approx} T_{\alpha}$.

4. Singular Measures: Error Rates. Now we study error rates under singular measures. When a measure is singular, the usual (Radon-Nikodym) density cannot be defined. Thus, we cannot define the $\lambda$-tree. However, as we discussed in Section 4 , we are still able to define the $\alpha$-tree. Thus, in this section, we will focus on error rates for the $\alpha$-tree.

4.1. Analysis of the KDE under Singular Measures. To study the convergence rate, we first investigate the bias of smoothing in the singular measure. Let $p_{h}(x)=\mathbb{E}\left(\widehat{p}_{n}\right)$, which is also known as the smoothed density.

\section{Assumption.}

(S) For all $s<d, \overline{\mathbb{K}}_{s}$ is a smooth manifold with positive reach and $\mathbb{K}$ is a compact set.

(P2) $\rho(x)$ is a generalized Morse function and there exists some $\rho_{\min }, \rho_{\max }>0$ such that $0<\rho_{\min } \leq$ $\rho(x) \leq \rho_{\max }<\infty$ for all $x$. Moreover, for any $s>0, \rho_{s}^{\dagger}$ is unique and has bounded continuous derivatives up to the fourth order.

Assumption $(\mathrm{S})$ ensures that $\mathbb{K}_{s}$ is smooth and every connected component of $\mathbb{K}_{s}$ is separated for each $s$. Assumption (P2) is a generalization of (P1) to singular distributions.

Lemma 6 (Bias of the smoothed density). Assume (S, PQ). Let $x \in \mathbb{K}_{s}$ and define $m(x)=$ $\min \left\{\ell>s: x \in \overline{\mathbb{K}_{\ell}}\right\}-s$. Let $C_{\ell}^{\dagger}=\left(\int_{B_{\ell}} K(\|y\|) d y\right)^{-1}$, where $B_{\ell}=\left\{y:\|y\| \leq 1, y_{\ell+1}=y_{\ell+2}=\cdots=\right.$ $\left.y_{d}=0\right\}$ for $\ell=1, \cdots, d$ and $d y$ is integrating with respect to $\ell$-dimensional area and $C_{0}^{\dagger}=1 / K(0)$. Then for a fixed $x$, when $h \rightarrow 0$ and $m(x)>0$,

$$
C_{\tau(x)}^{\dagger} h^{d-\tau(x)} \cdot p_{h}(x)=\rho(x)+\left\{\begin{array}{lll}
O\left(h^{2}\right)+O\left(h^{m(x)}\right), & \text { if } & m(x)>0 \\
O\left(h^{2}\right), & \text { if } & m(x)=0
\end{array} .\right.
$$


Moreover, if $\overline{\mathbb{K}_{\ell}} \cap \mathbb{K}_{s} \neq \phi$, for some $s<\ell$, then there exists $\epsilon>0$ such that

$$
\lim _{h \rightarrow 0} \sup _{x \in \mathbb{K}}\left|C_{\tau(x)} h^{d-\tau(x)} \cdot p_{h}(x)-\rho(x)\right|>\epsilon>0 .
$$

Lemma 6 describes the bias of the KDE. The scaling factor $C_{\tau(x)}^{\dagger} h^{d-\tau(x)}$ rescales the smoothed density to make it comparable to the generalized density. The first assertion is a pointwise convergence of smoothed density. In the case of $m(x)>0$, the bias contains two components: $O\left(h^{2}\right)$, the usual smoothing bias, and $O\left(h^{m(x)}\right)$, the bias from a higher dimensional support. This is because the $\mathrm{KDE}$ is isotropic, so the probability content outside $\mathbb{K}_{s}$ will also be included, which causes additional bias. The second assertion states that the smoothed density does not uniformly converge to the generalized density $\rho(x)$, so together with the first assertion, we conclude that the smoothing bias converges pointwisely but not uniformly. Next, we provide an example showing the failure of uniform convergence of a singular measure.

EXAMPLE 2 (Failure of uniform convergence). We consider $X$ from the same distribution as in Figure 2: with a probability of $0.3, X=2$, and with a probability of $0.7, X$ follows a standard normal. For simplicity, we assume that the kernel function is the spherical kernel $K(x)=\frac{1}{2} I(0 \leq x \leq 1)$ and consider the smoothing bandwidth $h \rightarrow 0$. This choice of kernel yields $C_{1}^{\dagger}=1$. Now consider a sequence of points $x_{h}=2+\frac{h}{2}$. Then the smoothed density at each $x_{h}$ is

$$
\begin{aligned}
p_{h}\left(x_{h}\right) & =\frac{1}{h} P\left(x_{h}-h<X<x_{h}+h\right) \\
& =\frac{1}{h} P\left(2-\frac{h}{2}<X<2+\frac{3 h}{2}\right) \\
& \geq \frac{1}{h} P(X=2)=\frac{3}{10 h},
\end{aligned}
$$

which diverges when $h \rightarrow 0$. However, it is easy to see that $\tau\left(x_{h}\right)=1$ and $\rho\left(x_{h}\right)=\frac{7}{10} \phi\left(x_{h}\right) \rightarrow \frac{7}{10} \phi(1)$ which is a finite number. Thus, $\mid \mathbb{E}\left(p_{h}\left(x_{h}\right)-\rho\left(x_{h}\right) \mid\right.$ does not converge.

REMARK 4. The scaling factor in Lemma $6 C_{\tau(x)}^{\dagger} h^{d-\tau(x)}$ depends on the support $\mathbb{K}_{s}$ where $x$ resides. In practice, we do not know $\tau(x)$ so we cannot properly rescale $\widehat{p}_{n}(x)$ to estimate $\rho(x)$. However, we are still able to rank pairs of data points based on Lemma 6 . To see this, let $x_{1}$ and $x_{2}$ be the two points that we want to compare their orderings (i.e., we want to know $x_{1} \prec_{\tau, \rho} x_{2}$ or $x_{1} \succ_{\tau, \rho} x_{2}$ or $\left.x_{1} \simeq_{\tau, \rho} x_{2}\right)$. When $x_{1}$ and $x_{2}$ are both in $\mathbb{K}_{s}$ for some $s$, the scaling does not affect the ranking between them so the sign of $\rho\left(x_{1}\right)-\rho\left(x_{2}\right)$ is the same as that of $p_{h}\left(x_{1}\right)-p_{h}\left(x_{2}\right)$. When $x_{1}$ and $x_{2}$ are in different supports (i.e. $x_{1} \in \mathbb{K}_{s_{1}}, x_{2} \in \mathbb{K}_{s_{2}}$, where $\left.s_{1} \neq s_{2}\right), p_{h}\left(x_{1}\right)$ and $p_{h}\left(x_{2}\right)$ diverge at different rates, meaning that we can eventually distinguish them. Thus, ordering for most points can still be recovered under singular measures. This is an important property that leads to the consistency of $\widehat{\alpha}_{n}$ under other error measurements.

Due to the failure of uniform convergence in the bias, the $L_{\infty}$ error of $\widehat{\alpha}_{n}$ does not converge under singular measures. 

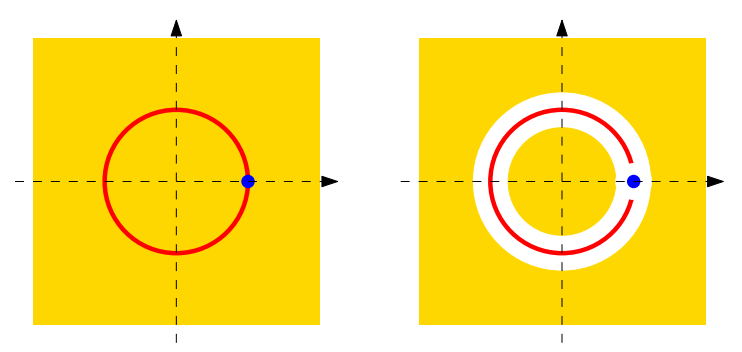

FIG 5. Good regions $\mathbb{K}_{s}(h)$ for Example 1. Left: the original $\mathbb{K}_{2}, \mathbb{K}_{1}$, and $\mathbb{K}_{0}$. Right: the corresponding $\mathbb{K}_{2}(h), \mathbb{K}_{1}(h)$, and $\mathbb{K}_{0}(h)$. The yellow area is the set $\mathbb{K}_{2}(h)$, which are regions in $\mathbb{K}_{2}$ away from lower-dimensional supports $\mathbb{K}_{1}$ and $\mathbb{K}_{0}$. The red area is the set $\mathbb{K}_{1}(h)$, which are regions in $\mathbb{K}_{1}$ where regions close to $\mathbb{K}_{0}$ have been removed. The blue dot is $\mathbb{K}_{0}(h)$, which is the same as $\mathbb{K}_{0}$ because there is no lower dimensional support. Note that $\mathbb{K}(h)$ is the union of the three color regions in the right panel.

Corollary 7 ( $L_{\infty}$ error for singular measures). Assume (S, P2). When $\overline{\mathbb{K}_{d}} \cap \overline{\mathbb{K}_{s}} \neq \phi$, for some $s<d,\left\|\widehat{\alpha}_{n}-\alpha\right\|_{\infty}$ does not converge to 0 . Namely, there exists $\epsilon>0$ such that

$$
\liminf _{n, h} P\left(\left\|\widehat{\alpha}_{n}-\alpha\right\|_{\infty}>\epsilon\right)>0
$$

The proof of Corollary 7 is a direct application of the failure of uniform convergence in smoothing bias shown in Lemma 6 . This corollary shows that for a singular measure, the $L_{\infty}$ error of the estimator $\widehat{\alpha}_{n}$ does not converge in general. Thus, there is no guarantee for the Hartigan consistency of the estimated $\alpha$-tree.

4.2. Error Measurements. Although Corollary 7 presents a negative result on estimating the $\alpha$ tree, in this section, we show that the estimator $\widehat{\alpha}_{n}$ is still consistent under other error measurements. A key observation is that there is a good region where the scaled KDE converges uniformly.

Let $\mathbb{K}_{s}(h)=\mathbb{K}_{s} \backslash\left(\bigcup_{\ell<s} \mathbb{K}_{\ell} \oplus h\right)$ be the set that is in the interior of $\mathbb{K}_{s}$ and is away from lower dimensional supports for $s>0$; in the case of $s=0$, we define $\mathbb{K}_{0}(h)=\mathbb{K}_{0}$. We define $\mathbb{K}(h)=$ $\bigcup_{s \leq d} \mathbb{K}_{s}(h)$, which is the union of each $\mathbb{K}_{s}(h)$. Figure 5 shows the good region of support $\mathbb{K}_{s}(h)$ and the original support $\mathbb{K}_{s}$ in Example 1. Later we will show that the set $\mathbb{K}(h)$ is the good region.

In Lemma 6, the quantity

$$
m(x)=\min \left\{\ell \geq \tau(x): x \in \overline{\mathbb{K}_{\ell}}\right\}-\tau(x)
$$

plays a key role in determining the rate of smoothing bias. Only when $m(x)=1$, do we have a slower rate for the bias. Thus, to obtain a uniform rate on the bias, we introduce the quantity

$$
m_{\min }=\inf _{x \in \mathbb{K}, m(x)>0} m(x) .
$$

If $m(x)=0$ for all $x \in \mathbb{K}$, we define $m_{\min }=2$. 
TheOrem 8 (Consistency of the KDE under singular measures). Assume (S, P2, K1-2). Let $C_{\ell}^{\dagger}$ be the constant in Lemma 6. Define

$$
\begin{aligned}
\delta_{n, h, s} & =\sup _{x \in \mathbb{K}_{s}(h)}\left\|C_{s}^{\dagger} h^{d-s} \widehat{p}_{n}(x)-\rho(x)\right\| \\
\delta_{n, h, s}^{(1)} & =\sup _{x \in \mathbb{K}_{s}(h)}\left\|C_{s}^{\dagger} h^{d-s} \nabla_{T_{s}(x)} \widehat{p}_{n}(x)-\nabla_{T_{s}(x)} \rho(x)\right\|_{\max } \\
\delta_{n, h, s}^{(2)} & =\sup _{x \in \mathbb{K}_{s}(h)}\left\|C_{s}^{\dagger} h^{d-s} \nabla_{T_{s}(x)} \nabla_{T_{s}(x)} \widehat{p}_{n}(x)-\nabla_{T_{s}(x)} \nabla_{T_{s}(x)} \rho(x)\right\|_{\max }
\end{aligned}
$$

where $\nabla_{T_{s}(x)}$ is taking gradient with respect to the tangent space of $\mathbb{K}_{s}$ at $x$. Then when $h \rightarrow$ $0, \frac{n h^{d+4}}{\log n} \rightarrow \infty$,

$$
\begin{aligned}
& \delta_{n, h, s}=O\left(h^{2 \wedge m_{\min }}\right)+O_{P}\left(\sqrt{\frac{\log n}{n h^{s}}}\right), \\
& \delta_{n, h, s}^{(1)}=O\left(h^{2 \wedge m_{\min }}\right)+O_{P}\left(\sqrt{\frac{\log n}{n h^{s+2}}}\right), \\
& \delta_{n, h, s}^{(2)}=O\left(h^{2 \wedge m_{\min }}\right)+O_{P}\left(\sqrt{\frac{\log n}{n h^{s+4}}}\right),
\end{aligned}
$$

where $a \bigwedge b=\min \{a, b\}$.

Theorem 8 shows that after rescaling, the KDE is uniformly consistent within the good region $\mathbb{K}_{s}(h)$ for density, gradient, and Hessian estimation. A more interesting result is that, after rescaling, the error rate is the same as the usual $L_{\infty}$ error rate in the $s$-dimensional case with a modified bias term (bias is affected by a higher dimensional support).

REMARK 5. (Non-convergence of the integrated distance of the KDE) One may wonder if the scaled KDE $\left(C_{\tau(x)}^{\dagger} h^{d-\tau(x)} \cdot \widehat{p}_{n}(x)\right)$ converges to the generalized density $\rho(x)$ under the integrated distance. There is no guarantee for such a convergence because

$$
\int\left\|C_{\tau(x)}^{\dagger} h^{d-\tau(x)} \cdot \widehat{p}_{n}(x)-\rho(x)\right\| d x=O_{P}(1) .
$$

To see this, consider a point $x \in \mathbb{K}_{s}$ and let $\mathbb{K}_{\ell}$ be a higher order support $(\ell>s)$ with $x \in \overline{\mathbb{K}_{\ell}}$. Then the region $B(x, h) \cap \mathbb{K}_{\ell}$ has $\ell$-dimensional volume at rate $O\left(h^{\ell-s}\right)$. For any point $y \in B(x, h) \cap \mathbb{K}_{\ell}$, $\tau(y)=\ell$ but the $\operatorname{KDE} \widehat{p}_{n}(y)$ is at rate $O_{P}\left(h^{s-d}\right)$. Thus, the difference between the scaled KDE and the generalized density is

$$
C_{\ell}^{\dagger} h^{d-\ell} \cdot \widehat{p}_{n}(y)-\rho(y)=O_{P}\left(h^{s-\ell}\right) .
$$

Such $y$ has $\ell$-dimensional volume at rate $O\left(h^{\ell-s}\right)$, so the integrated error is at rate $O_{P}\left(h^{s-\ell}\right) \times$ $O\left(h^{\ell-s}\right)=O_{P}(1)$.

Based on Theorem 8, we can derive a nearly uniform convergence rate of $\widehat{\alpha}_{n}$. 
TheOrem 9 (Nearly uniform consistency of $\alpha$-trees). Assume (S, P2, K1-2). Let $\mathcal{C}_{s}$ be the collection of generalized critical points of $\mathbb{K}_{s}$. Let $\delta_{n, h, s}$ be defined in equation (11) and $r_{n, h, s}$ be a quantity such that $\frac{\delta_{n, h, s}}{r_{n, h, s}}=o_{P}(1)$. Then when $h \rightarrow 0, \frac{n h^{d+2}}{\log n} \rightarrow \infty$, uniformly for every $x \in \mathbb{K}_{s}(h)$,

$$
\widehat{\alpha}_{n}(x)-\alpha(x)= \begin{cases}O\left(\delta_{n, h, s}\right) & \text { if } \inf _{c \in \mathcal{C}_{s}}|\rho(x)-\rho(c)|>r_{n, h, s}, \\ O\left(\left(\delta_{n, h, s}\right)^{\frac{s}{s+1}}\right) & \text { otherwise }\end{cases}
$$

The convergence rate in Theorem 9 is similar to that in Theorem 8: for a given point $x$ when $\alpha(x)$ is away from the $\alpha$ value of a generalized critical point (a critical $\alpha$ level), we have the usual convergence rate. When $\alpha(x)$ is close to a critical $\alpha$ level, the convergence rate is slower. The quantity $r_{n, h, s}$ behaves like the quantity $\varpi_{n}$ in Theorem 4 , which was introduced to guarantee the uniform convergence. To derive the consistency of $\widehat{\alpha}_{n}$ under the integrated error and the probability error, we choose $r_{n, h, s}=\frac{\delta_{n, h, s}}{\log n}$, which leads to the following theorem.

Theorem 10 (Consistency of $\alpha$-trees). Assume (S, P2 , K1-2).

Then

$$
\begin{aligned}
\left\|\widehat{\alpha}_{n}-\alpha\right\|_{P} & =O\left(\delta_{n, h, d}\right), \\
\left\|\widehat{\alpha}_{n}-\alpha\right\|_{\mu} & =O\left(\delta_{n, h, d}\right) .
\end{aligned}
$$

Theorem 9 shows that the quantity $\alpha(x)$ can be consistently estimated by $\widehat{\alpha}_{n}(x)$ for the majority points. This implies that the ordering of points using $\widehat{p}_{n}$ is consistent with the ordering from $\tau, \rho$ in most areas of $\mathbb{K}$.

5. Singular Measures: Critical Points and Topology. Recall from Section 2.1 that the topology of an $\alpha$-tree $T_{\alpha}$ is determined by its edge set $E\left(T_{\alpha}\right)$ and the relation among edges $\mathbb{C} \in$ $E\left(T_{\alpha}\right)$. The set $\mathcal{A}_{\alpha}$ (critical tree-levels) contains the levels where the upper level set $\mathbb{A}_{\varpi}=\{x:$ $\alpha(x) \geq \varpi\}$ changes its shape. For nonsingular measures, $\mathcal{A}_{\alpha}$ corresponds to the density value of some critical points. For singular measures, this is not true even when $\rho(x)$ is a generalized Morse function.

Consider the example in Figure 6. The solid box in the left panel indicates a new type of critical points, where merges between elements in different edge sets occur (change in the topology of level sets occurs). By the definition of $\mathcal{A}_{\alpha}$, this corresponds to an element in $\mathcal{A}_{\alpha}$, but it is clearly not a generalized critical point. We call this type of critical points the dimensional critical points (DCPs). In Figure 6, the dimension $d=2$ and we have a $2 D$ spherical distribution mixed with a $1 D$ singular measure that is distributed on the red curves $\mathbb{K}_{1}$. The bluish contours are density contours of the $2 D$ spherical distribution, the crosses are locations of local modes, and the solid box is the location of a DCP. To see how the solid box changes the topology of level sets, we display two level sets in the middle and right panels. In the middle panel, the level is high and there are two connected components (the gray area and the solid curve). In the right panel, we lower the level, and now, the two connected components merge at the location of the solid box. Although the location of the solid box does not belong to the collection of generalized critical points $\mathcal{C}$, this point does correspond to 

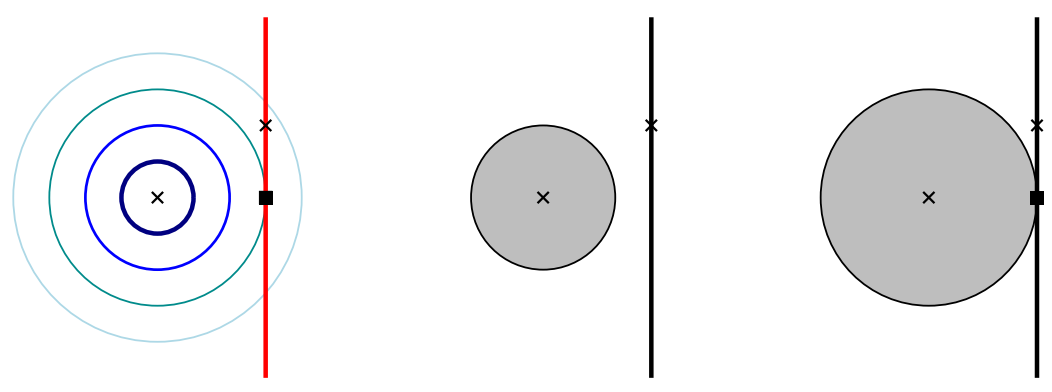

FIG 6. Example of DCPs. This is a $d=2$ case; there is a $2 D$ spherical distribution mixed with a $1 D$ distribution on a line segment. Left: the blue contours are density contours of the $2 D$ spherical distribution and the red line segment is $\mathbb{K}_{1}$, the support of the $1 D$ singular distribution. The two crosses are the density maxima at the $2 D$ distribution and the $1 D$ singular distribution. The black square indicates a DCP. To see how DCPs merge two connected components, we consider the middle and the right panel, which are level sets of $\alpha(x)$ at two different levels. Middle: the level set $\mathbb{A}_{\varpi}$ where the level $\varpi$ is high; we can see that there are two connected components (left gray-black disk and the right line segment). Right: we move down the level a little bit; now the two connected components merge so there is only one connected component. The merging point is the square point, which is defined as a DCP.

the merging of connected components in the level sets. Thus, this point corresponds to an element in $\mathcal{A}_{\alpha}$.

Here is the formal definition of the DCP. Recall that $\mathcal{C}$ is the collection of generalized critical points of $\rho(x)$ and from equation $(1), \mathcal{A}_{\alpha}$ is the collection of levels of function $\alpha(x)$ such that the creation of a new connected component or merging of connected components occurs. For simplicity, we denote $\mathcal{A}=\mathcal{A}_{\alpha}$. For any level $\varpi \in \mathcal{A}$, we define

$$
\xi(\varpi)= \begin{cases}\max \left\{w \geq 0: \mathbb{K}_{w} \subset \mathbb{A}_{\varpi}\right\}, & \text { if } \mathbb{K}_{0} \subset \mathbb{A}_{\varpi} . \\ -1, & \text { if } \mathbb{K}_{0} \not \subset \mathbb{A}_{\varpi}\end{cases}
$$

Namely, $\xi(\varpi)$ is the highest dimensional structure that is covered by the level set $\mathbb{A}_{\varpi}$.

Definition 3. For $\varpi \in \mathcal{A}$, we say $x$ is a DCP if the following holds

(1) $x \in \mathbb{K}_{\ell}$ for some $\ell \leq \xi(\varpi)$.

(2) There exists an edge $\mathbb{C} \in E\left(T_{\alpha}\right)$ such that

(i) $x \notin C$ for all $C \in \mathbb{C}$,

(ii) $d\left(x, C_{\epsilon}\right) \rightarrow 0$ when $\epsilon \rightarrow 0$, where $C_{\epsilon}=\mathbb{C} \cap T_{\alpha}(\varpi+\epsilon)$.

Note that $x$ may not exist. In such a case, there is no DCPs for level $\varpi$.

The first requirement is to ensure that $x$ is on a lower dimensional support $\left(\mathbb{K}_{\ell}: \ell<\xi(\varpi)\right)$. The second requirement is to show that the DCP $x$ is not in the same edge $\mathbb{C}$, but its distance to the elements (connected components) of the edge is shrinking to 0. By the definition of $\alpha(x)$, the first requirement implies that $x$ is contained in $\mathbb{A}_{\varpi+\epsilon}$ for a sufficiently small $\epsilon$. Therefore, we can find $\mathbb{C}^{\prime} \in E\left(T_{\alpha}\right)$ such that every element $C \in \mathbb{C}^{\prime}$ contains $x$. Because $x$ is (i) in the elements of edge $\mathbb{C}^{\prime}$, and (ii) not in any element of edge $\mathbb{C}$, and because (iii) the distance from $x$ to the element of $\mathbb{C}$ 
converges to 0 when the level decreases to the level $\varpi, x$ is a merging point of edges $\mathbb{C}^{\prime}$ and $\mathbb{C}$ and the level $\varpi$ is their merging level.

Note that since DCPs occur when different dimensional regions intersect each other, the topological structure of such an intersection may be related to the intersection homology and stratified space (Edelsbrunner and Harer, 2008; Goresky and MacPherson, 1980, 2012).

Let $\mathcal{C}^{D}$ be the collection of DCPs. For a point $c \in \mathcal{C}^{D}$, we denote $\alpha^{\dagger}(c)$ as the level of $\alpha$ function corresponding to the DCP at $c$. Note that $\alpha^{\dagger}(c) \neq \alpha(c)$ when $c$ is a DCP. Since a DCP is generally in a lower dimensional support than the support that the merging happens, $\alpha(c)>\alpha^{\dagger}(c)$. Remark 7 provides an example of how $\alpha$ and $\alpha^{\dagger}$ differs.

REMARK 6 (Relation to the usual critical points). The definition of DCPs is similar to that of saddle points or local minima, who contribute to the merging of level sets. Saddle points (or local minima) that contribute to a merging of level sets can be defined as a point $x$ with the following properties:

(1) $x \in \mathbb{K}_{\xi(\varpi)+1}$.

(2) There exists two different edges $\mathbb{C}_{1}, \mathbb{C}_{2} \in E\left(T_{\alpha}\right)$ such that

(i) $x \notin C_{1}, x \notin C_{2}$ for all $C_{1} \in \mathbb{C}_{1}$ and $C_{2} \in \mathbb{C}_{2}$,

(ii) $d\left(x, C_{1, \epsilon}\right) \rightarrow 0$ when $\epsilon \rightarrow 0$, where $C_{1, \epsilon}=\mathbb{C}_{1} \cap T_{\alpha}(\varpi+\epsilon)$,

(iii) $d\left(x, C_{2, \epsilon}\right) \rightarrow 0$ when $\epsilon \rightarrow 0$, where $C_{2, \epsilon}=\mathbb{C}_{2} \cap T_{\alpha}(\varpi+\epsilon)$.

It is easy to see that for a Morse function, a point $x$ with the above properties must be a saddle point or a local minimum. The main difference between this definition and that of DCPs is the support where $x$ lives- if $x$ lives in a lower dimensional support $\mathbb{K}_{\ell}, \ell \leq \xi(\varpi)$, then it is a DCP, and if $x$ lives in the support $\mathbb{K}_{\xi(\varpi)+1}$, then it is a saddle point or a local minimum.

REMARK 7. Note that a DCP might be at the same position as a critical point. Consider the example in Figure 2 and Example 2. In this case,

$$
\alpha(x)=\left\{\begin{array}{ll}
0.7 \cdot 2 \cdot \Phi_{0}(-|x|), & \text { if } x \neq 2 \\
1, & \text { if } x=2
\end{array},\right.
$$

where $\Phi_{0}(x)$ is the cumulative distribution of a standard normal. Moreover, $\mathcal{A}_{\alpha}=\{1,0.7,0.0319\}$; the first element $\{1\}$ is the level of the point mass located at $x=2$, the second element $\{0.7\}$ is the level of the mode of the standard normal distribution, the last element is the level where the connected components created at levels 1 and 0.7 merged so it comes from a DCP. This DCP also located at $x=2$, which coincides with a local mode. Note that the number $0.0319=0.7 \cdot 2 \cdot \Phi_{0}(-2)$, which is the level where the merging occurred. At the critical point $x=2, \alpha(2)=1$ and $\alpha^{\dagger}(2)=$ 0.0319 .

To analyze the properties of DCPs and their estimators, we consider the following assumptions. Assumptions.

(A) The elements in the collection $\mathcal{A}$ are distinct and each element corresponds to one critical point or one DCP, but not both. And all DCPs are distinct.

(B) For every $x \in \partial \mathbb{K}_{s}(s>0)$ and $r>0$, there is $y \in B(x, r) \cap \mathbb{K}_{s}$ such that $\rho(y)>\rho(x)$. 
(C) There exists $\eta_{0}>0$ such that

$$
\inf _{c \in \mathcal{C}_{s}} d\left(c, \mathbb{K}_{\ell}\right) \geq \eta_{0}
$$

for all $\ell<s$ and $s=1,2, \cdots, d$.

Assumption (A) is to ensure that no multiple topological changes will occur at the same level so each level corresponds to only a merging or a creation. Assumption (B) is to guarantee that no new connected component at the boundary of a lower dimensional manifold will be created. Thus, any creation of a new connected component of the level set $\mathbb{A}_{\varpi}$ occurs only at a (generalized) local mode. Assumption (C) is to regularize (generalized) critical points so that they are away from lower dimensional supports. This implies that when $h$ is sufficiently small, all critical points will be in the $\operatorname{good}$ region $\mathbb{K}(h)$.

Lemma 11 (Properties of DCPs). Assume (S, PQ, B). The DCPs have the following properties

- If a new connected component of $\mathbb{A}_{\varpi}$ is created at $\varpi \in \mathcal{A}$, then there is a local mode $c$ of $\rho$ or an element in $\mathbb{K}_{0}$ such that $\varpi=\alpha(c)$. Namely, DCPs only merge connected components.

- For any value $\varpi \in \mathcal{A}$, either $\varpi=\alpha(c)$ for some $c \in \mathcal{C}$ or there is a DCP associated with $\varpi$.

Lemma 11 provides two basic properties of DCPs. First, DCPs only merge connected components. Moreover, when the topology of connected components of $\alpha$-level sets changes (when we decrease the level), either a critical point or a DCP must be responsible for this. Theorefore, as long as we control the stability of generalized critical points and DCPs, we control the topology of an $\alpha$-tree. Thus, in what follows, we will study the stability of generalized critical points and DCPs.

Lemma 12 (Stability of generalized critical points). Assume (S, P2, K1-2, C). Let $c \in \mathbb{K}_{s}$ be a generalized critical point with $n(c)$ negative eigenvalues of its generalized Hessian matrix. Let $\widehat{\mathcal{C}}$ be the collection of critical points of $\widehat{p}_{n}$. Then when $h \rightarrow 0, \frac{n h^{d+4}}{\log n} \rightarrow \infty$, there exists a point $\widehat{c} \in \widehat{\mathcal{C}}$ such that

$$
\begin{aligned}
\|\widehat{c}-c\| & =O(h)+O_{P}\left(\sqrt{\frac{1}{n h^{s+2}}}\right) \\
\left\|\widehat{\alpha}_{n}(\widehat{c})-\alpha(c)\right\| & =O_{P}\left(\left(\delta_{n, h, s}\right)^{\frac{s}{s+1}}\right)
\end{aligned}
$$

and the estimated Hessian matrix at $\widehat{c}$ has $n(c)+d-s$ negative eigenvalues. The quantity $\delta_{n, h, s}$ is defined in equation (11).

Lemma 12 is a generalization of the stability theorem of critical points given in Lemma 16 of Chazal et al. (2014). Note that the bias is now of the order $O(h)$; this is due to the smoothing effect from a higher dimensional support.

Lemma 13 (Properties of estimated critical points). Assume (S, P2, K1-2, A, B). Assume there are $k$ DCPs. Let $\widehat{\mathcal{C}}$ be the critical points of $\widehat{p}_{n}$. Define $\widehat{\mathcal{G}} \subset \widehat{\mathcal{C}}$ as the collection of estimated critical

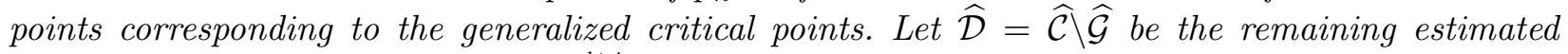
critical points. Then when $h \rightarrow 0, \frac{n h^{d+4}}{\log n} \rightarrow \infty$,

- $\widehat{\mathcal{D}} \subset \mathbb{K}^{C}(h)$, 
- $|\widehat{\mathcal{D}}| \geq k$, where $|A|$ for a set $A$ is the cardinality,

- $\widehat{\mathcal{D}}$ contains no local mode of $\widehat{p}_{n}$.

Lemma 13 provides several useful properties of the estimated critical points (critical points of $\left.\widehat{p}_{n}\right)$. First, the estimated critical points are all in the bad region $\mathbb{K}^{C}(h)$, except for those converging to generalized critical points. Second, the number of estimated critical points will (asymptotically) not be less than the total number of DCPs. Third, all estimated local modes are estimators of generalized critical points.

Lemma 14 (Stability of critical tree-levels from DCPs). Assume (S, P2, K1-2, A, B). Let $c$ be a DCP and $\alpha^{\dagger}(c) \in \mathcal{A}$ be the associated level. Let $\widehat{\mathcal{D}}$ be defined as Lemma 13. Then when $h \rightarrow 0, \frac{n h^{d+2}}{\log n} \rightarrow \infty$, there exists a point $\widehat{c} \in \widehat{\mathcal{D}}$ such that

$$
\left\|\widehat{\alpha}_{n}(\widehat{c})-\alpha^{\dagger}(c)\right\|=O\left(\delta_{n, h, \xi\left(\alpha_{0}(c)\right)+1}\right),
$$

where $\delta_{n, h, s}$ is defined in (11). Moreover, the $\widehat{\mathbb{A}}_{\widehat{\alpha}_{n}(\widehat{c})+\epsilon}$ and $\widehat{\mathbb{A}}_{\widehat{\alpha}_{n}(\widehat{c})}$ are not topological equivalent.

Lemma 14 illustrates the stability of critical tree-levels from DCPs: for every DCP, there will be an estimated critical point that corresponds to this DCP and this estimated critical point also represents a merging of the estimated level sets.

In Lemma 12, we derived the convergence rate of the estimated (generalized) critical points versus the population critical points, but here we only derive the rate for the critical tree-levels. The reason is that critical points are solutions to a certain function (gradient equals to 0), so we can perform a Taylor expansion to obtain the convergence rate. However, for the DCPs, they are not solutions to some functions, so it is unclear how to derive the convergence rate for their locations.

EXAmPle 3 (A DCP and its estimator). Consider again the example in Figure 2 and Example 2. We have a singular distribution mixed with a point mass at $x=2$ with a probability of 0.3 and a standard normal with a probability of 0.7 . In this case, as indicated, a DCP is located at $x=2$ with level 0.0319 (see Remark 7). In every panel of the top row of Figure 2, there is a local minimum located in the region $x \in[1.5,2]$. Moreover, when we increase the sample size (from left to right), this local minimum is moving toward $x=2$. This local minimum is an estimated critical point $\widehat{c} \in \widehat{\mathcal{D}}$, as described in Lemma 14, whose estimated $\alpha$-level is approaching the $\alpha$-level of the DCP at $x=2$.

Theorem 15 (Topological error of $\alpha$-trees). Assume (S, P2, K1-2, A, B, C). Then when $h \rightarrow$ $0, \frac{n h^{d+4}}{\log n} \rightarrow \infty$,

$$
P\left(T_{\widehat{\alpha}_{n}} \stackrel{T}{\approx} T_{\alpha}\right) \geq 1-c_{0} \cdot e^{-c_{1} \cdot n h^{d+4}}
$$

for some $c_{0}, c_{1}>0$.

Theorem 15 quantifies the topological error of the estimated $\alpha$-tree under singular measures. The error rate is the same as that in the nonsingular measures (Theorem 5). The topological error bound is similar to that in Corollary 5. Both have exponential concentration bounds with a factor of $n h^{d+4}$, which is the Hessian estimation error rate. The two concentration bounds are similar, because as 
shown in Theorem 8, the main difference between singular and nonsingular measures lies in the bias part, which will not contribute to the concentration inequality as long as $h \rightarrow 0$. The Hessian error rate is because we need to make sure the signs of eigenvalues of Hessian matrices around critical points remain unchanged.

REMARK 8. Theorem 15 also implies that, under singular measures, the cluster tree of the KDE $\widehat{p}_{n}$ (estimated $\lambda$-tree) converges topologically to a population cluster tree defined by the function $\alpha(x)$. To see this, recall that by Lemma $1, T_{\widehat{p}_{n}} \stackrel{T}{\approx} T_{\widehat{\alpha}_{n}}$. This, together with Theorem 15, implies

$$
P\left(T_{\widehat{p}_{n}} \stackrel{T}{\approx} T_{\alpha}\right) \geq 1-c_{0} \cdot e^{-c_{1} \cdot n h^{d+4}} \rightarrow 1
$$

under a suitable choice of $h$. This shows that even when the population distribution is singular, the estimated $\lambda$-tree still converges topologically to the population $\alpha$-tree.

6. Discussion. In this paper, we study how the $\alpha$-tree behaves under singular and nonsingular measures. In the nonsingular case, the error rate under the $L_{\infty}$ metric is slower than other metrics because of the slow rate of level set estimation around saddle points. However, other error rates are the same as estimating the $\lambda$-tree.

When a distribution is singular, we obtain many fruitful results for both the KDE and the estimated $\alpha$-tree. In terms of the KDE, we prove that

1. the KDE is a pointwise consistent estimator after rescaling;

2. the KDE is a uniformly consistent estimator after rescaling for the majority of the support; and

3. the cluster tree from the $\mathrm{KDE}$ (estimated $\lambda$-tree) converges topologically to a population cluster tree defined by $\alpha$.

For the estimator $\widehat{\alpha}_{n}(x)$ and the estimated $\alpha$-tree, we show that

1. $\widehat{\alpha}_{n}$ is a pointwise consistent estimator of $\alpha$;

2. $\widehat{\alpha}_{n}$ is a uniformly consistent estimator for the majority of the support;

3. $\widehat{\alpha}_{n}$ is a consistent estimator of $\alpha$ under the integrated distance and probability distance; and

4. the estimated $\alpha$-tree converges topologically to the population $\alpha$-tree.

Moreover, we observe a new type of critical points-the DCPs-that also contribute to the merging of level sets for singular measures. We study the properties of DCPs and show that the estimated critical points from the KDE approximate these DCPs.

Finally, we point out some possible future directions.

- Persistence Homology. The cluster tree is closely related to the persistent homology of level sets (Fasy et al., 2014; Bobrowski et al., 2017). In the persistent homology, a common metric for evaluating the quality of an estimator is the bottleneck distance (Cohen-Steiner et al., 2007; Edelsbrunner and Morozov, 2012). Because the bottleneck distance is bounded by the $L_{\infty}$ metric (Cohen-Steiner et al., 2007; Edelsbrunner and Morozov, 2012), many bounds on the bottleneck distance are derived via bounding the $L_{\infty}$ metric (Fasy et al., 2014; Bobrowski et al., 2017). However, for $\alpha$-trees under singular measures, the $L_{\infty}$ metric does not converge (Corollary 7) but we do have topological consistency (Theorem 15), which implies convergence in the bottleneck distance. This provides an example where we have consistency under the 
bottleneck distance and inconsistency in the $L_{\infty}$ metric. How this phenomenon affects the persistence homology is unclear and we leave that line of study for future work.

- Higher-order Homology Groups and Stratified Space. Our definition of DCPs is for connected components, which are zeroth-order homology groups (Cohen-Steiner et al., 2007; Fasy et al., 2014; Bubenik, 2015) and sufficient for analyzing cluster trees. However, critical points also contribute to the creation and elimination of higher-order homology groups such as loops and voids, which are not covered in this paper. Thus, a future direction is to study whether the KDE is also consistent in recovering higher-order homology groups under singular measures. Moreover, as is mentioned in Remark 2, the supports we are analyzing are related to the stratified space (Friedman, 2014; Goresky and MacPherson, 1980) and the DCPs might be related to the intersection homology (Friedman, 2014; Goresky and MacPherson, 1980; Edelsbrunner and Harer, 2008). The intersection homology extends the definition of homology group to a stratified space so it provides a tool to analyze higher-order homology groups in our setting. Thus, finding the connection between theories of stratified space and the higher-order homology groups in our settings will be another future research direction.

- Minimax theory. Chaudhuri and Dasgupta (2010); Chaudhuri et al. (2014) derived the minimax theory for estimating the $\lambda$-tree under nonsingular measures and proved that the the k-nearest neighbor estimator is minimax. When the distribution is nonsingular, the $\alpha$-tree and $\lambda$-tree are very similar so we expect the minimax theory to be the same. However, for singular measures, it is unclear how to derive the minimax theory so we plan to investigate this in the future.

Acknowledgements. We thank reviewers for their very insightful comments. We also thank members in the CMU Topstat group for useful discussion.

\section{APPENDIX A: PROOF}

We use $g$ to denote the gradient of $p$ (when it exists) and $\widehat{g}_{n}$ to denote the gradient of $\widehat{p}_{n}$.

To prove Theorem 3, we will use the following lemma.

Lemma 16. Assume $p$ satisfies (P1). Let $\lambda$ be the density value of a critical point and $\mathcal{C}(\lambda)$ be the collection of all critical points with density value being $\lambda$. Let $\partial L_{\lambda}=\{x: p(x)=\lambda\}$. Then there exists $\bar{R}>0$ and $0<C_{\min } \leq C_{\max }<\infty$ such that for any positive number $r<\bar{R}$,

$$
\begin{aligned}
& \inf _{x \in\left(\partial L_{\lambda} \oplus \bar{R}\right) \backslash(\mathcal{C}(\lambda) \oplus r)}\|g(x)\| \geq C_{\min } r, \\
& \sup _{x \in \mathcal{C}(\lambda) \oplus r}\|g(x)\| \leq C_{\max } r .
\end{aligned}
$$

The first assertion means that the low gradient region must be around critical points and the gradient has to be linearly increasing as we move away from a critical point. The second assertion states that when we move away from a critical point, the gradient cannot increase faster than a linear rate. Essentially, these two properties are just natural outcomes from the fact that the density $p$ is a Morse function (Hessian matrix at critical points have non-zero eigenvalues) and has bounded third derivatives. 
Proof. First assertion. By the continuity of the gradient of $p$ (assumption (P1) implies bounded Hessian matrix, which implies the continuity of gradient), regions with low gradient must be around a critical point.

Let $c_{0}$ be a critical point of $\mathcal{C}(\lambda)$. When $\bar{R}$ is sufficiently small, for any unit vector $\nu \in \mathbb{R}^{d}$ and any two positive numbers $r_{1}<r_{2}<\bar{R}$, we have

$$
0<\left\|g\left(c_{0}+r_{1} \nu\right)\right\|<\left\|g\left(c_{0}+r_{2} \nu\right)\right\| .
$$

Namely, if we are moving away from a critical point along a fixed orientation, the amount gradient will be increasing as long as we do not move too far away. This is because the density around a critical point behaves like a quadratic function due to the Morse lemma (see, e.g., Lemma 3.11 of Banyaga and Hurtubise 2013). Thus, when $\bar{R}$ is sufficiently small and $r<\bar{R}$, the infimum $\inf _{x \in\left(\partial L_{\lambda} \oplus \bar{R}\right) \backslash(\mathcal{C}(\lambda) \oplus r)}\|g(x)\|$ occurs at the boundary $\partial(\mathcal{C}(\lambda) \oplus r)$. Let $x_{0} \in \partial(\mathcal{C}(\lambda) \oplus r)$ be the point where the infimum occurs and $c$ be the critical point of $\mathcal{C}(\lambda)$ such that $\left\|x_{0}-c\right\|=r$.

Because $p$ is a Morse function, all eigenvalues of $H^{2}(c)$ must be non-zero. Thus, the fact that $p$ has a bounded third derivative (assumption (P1)) implies that when $\bar{R}$ is sufficiently small, all eigenvalues of $H^{2}(x)$ must be greater than or equal to a positive constant $C_{1}$ uniformly for all $x \in B(c, \bar{R})$. Define a line $L_{0}(t)=t \cdot x_{0}+(1-t) \cdot c$ passing through $c$ and $x_{0}$. Then the gradient

$$
\begin{aligned}
g\left(x_{0}\right)= & \underbrace{g(c)}_{=0(c \text { is a critical point })}+\int_{t=0}^{t=1} d g\left(L_{0}(t)\right) \\
= & \int_{t=0}^{t=1} H\left(L_{0}(t)\right) \underbrace{L_{0}^{\prime}(t)}_{=x_{0}-c} d t,
\end{aligned}
$$

which implies

$$
\left\|g\left(x_{0}\right)\right\|=\left\|\int_{t=0}^{t=1} H\left(L_{0}(t)\right)\left(x_{0}-c\right) d t\right\| \geq \sqrt{C_{1}}\left\|x_{0}-c\right\|=C_{\min } r,
$$

which proves the first assertion.

Second assertion. By assumption (P1), every element of $H(x)$ is uniformly bounded so the eigenvalues of $H^{2}(x)$ is also bounded. Let $C_{\max }=\sup _{x}\|H(x)\|_{*}$ be the uniform bound on the spectral norm of $H(x)$. For any point $x \in B(c, r)$, again we define the line $L_{0}(t)=t \cdot x_{0}+(1-t) \cdot c$ passing through $c$ and $x_{0}$. Then,

$$
\begin{aligned}
g\left(x_{0}\right)= & \underbrace{g(c)}+\int_{t=0}^{t=1} d g\left(L_{0}(t)\right) \\
= & \int_{t=0}^{t=1} H\left(L_{0}(t)\right) \underbrace{L_{0}^{\prime}(t)}_{=x_{0}-c} d t
\end{aligned}
$$

and

$$
\left\|g\left(x_{0}\right)\right\|=\left\|\int_{t=0}^{t=1} H\left(L_{0}(t)\right)\left(x_{0}-c\right) d t\right\| \leq C_{\max }\|x-c\| \leq C_{\max } r,
$$

which completes the proof. 
Proof of Theorem 3. Let $c$ be a critical point with $\lambda=p(c)$. We denote $\delta_{\infty}=\left\|\widehat{p}_{n}-p\right\|_{\infty}$. For a point $y \in \widehat{L}_{\lambda}$,

$$
\lambda \leq \widehat{p}_{n}(y) \leq p(y)+\delta_{\infty} \quad \Rightarrow \quad p(y) \geq \lambda-\delta_{\infty} .
$$

Thus, for a point $y \in \widehat{L}_{\lambda} \backslash L_{\lambda}$, we have

$$
\lambda>p(y) \geq \lambda-\delta_{\infty} .
$$

Similarly, for any point $y \in L_{\lambda} \backslash \widehat{L}_{\lambda}$, we have

$$
\lambda+\delta_{\infty}>p(y) \geq \lambda
$$

Thus, the set difference

$$
\left(\widehat{L}_{\lambda} \triangle L_{\lambda}\right) \subset D_{\lambda, \infty}=\left\{x:|p(x)-\lambda| \leq \delta_{\infty}\right\}
$$

which further implies

$$
\mu\left(\widehat{L}_{\lambda} \triangle L_{\lambda}\right) \leq \mu\left(D_{\lambda, \infty}\right) .
$$

So we can bound the quantity $\mu\left(D_{\lambda, \infty}\right)$ to complete the proof.

Note that by the continuity of $p(x)$ and the fact that $p$ is a Morse function (implying no flat density region), $\delta_{\infty} \rightarrow 0$ implies that $\operatorname{Haus}\left(D_{\lambda, \infty}, \partial L_{\lambda}\right) \rightarrow 0$, where $\partial L_{\lambda}=\{x: p(x)=\lambda\}$ is the boundary of $L_{\lambda}$. Thus, Lemma 16 holds when $\delta_{\infty}$ is sufficiently small.

We partition the set $D_{\lambda, \infty}$ into two regions:

$$
\begin{aligned}
& A_{n}=D_{\lambda, \infty} \bigcap B^{C}\left(c, R_{n}\right), \\
& B_{n}=D_{\lambda, \infty} \bigcap B\left(c, R_{n}\right),
\end{aligned}
$$

where $R_{n}$ is a number that shrinks to 0 when $\delta_{\infty} \rightarrow 0$. Later we will provide an explicit expression for $R_{n}$. We only consider the case where $c$ is unique and is a saddle point; one can easily generalize the proof to the case of multiple critical points because $p$ is a Morse function so every critical point is well-separated (see, e.g., Lemma 3.2 of Banyaga and Hurtubise 2013).

The proof requires two simple geometric facts:

- Fact 1: Let $\gamma_{y}(t)$ be a curve starting from $y$ passing through a point $x$ at time $t_{x}$. Namely, $\gamma_{y}(0)=y$ and $\gamma_{y}\left(t_{x}\right)=x$. Then

$$
\|x-y\| \leq \int_{0}^{t_{x}}\left\|\gamma_{y}^{\prime}(t)\right\| d t,
$$

i.e, the distance between two points is less than or equal to the length of any curve connecting the two points.

- Fact 2: If $\partial L_{\lambda}$ has finite $(d-1)$-dimensional volume (i.e., finite surface area), then when $r_{n} \rightarrow 0$,

$$
\mu\left(\partial L_{\lambda} \oplus r_{n}\right)=O\left(r_{n}\right)
$$

where $\mu(\cdot)$ is the Lebesgue measure. Note that this fact is because $p$ is a Morse function so the level set $\partial L_{\lambda}=\{x: p(x)=\lambda\}$ is a $(d-1)$ rectifiable set and thus the Minkowski content $\left(\lim _{\epsilon \rightarrow 0} \frac{\mu\left(\partial L_{\lambda} \oplus \epsilon\right)}{2 \epsilon}\right)$ equals to the $(d-1)$-dimensional volume. 
For simplicity, we first consider the points in $A_{n}$ with a density value below $\lambda$. Let $x \in A_{n} \cap\{y$ : $p(y)<\lambda$ \} be one such point. This implies $p(x)<\lambda$. Define a density gradient (ascent) flow $\pi_{x}$ starting at $x$ as follows:

$$
\pi_{x}(0)=0, \quad \pi_{x}^{\prime}(t)=g\left(\pi_{x}(t)\right)
$$

Namely, the gradient flow $\pi_{x}$ is a flow starting at $x$ that follows the gradient of the density function. Because this flow follows the density gradient, the density along this flow $p\left(\pi_{x}(t)\right)$ is increasing with respect to $t$. Let $t_{0}$ be the time such that $p\left(\pi_{x}\left(t_{0}\right)\right)=\lambda$. Then we have

$$
\begin{aligned}
\lambda-p(x) & =\int_{0}^{t_{0}}\left(\frac{d p\left(\pi_{x}(t)\right)}{d t}\right) d t \\
& =\int_{0}^{t_{0}} g^{T}\left(\pi_{x}(t)\right) \pi_{x}^{\prime}(t) d t=\int_{0}^{t_{0}}\left\|g\left(\pi_{x}(t)\right)\right\|^{2} d t
\end{aligned}
$$

because $\pi_{x}^{\prime}(t)=g\left(\pi_{x}(t)\right)$ by construction. By the definition of $A_{n}, \lambda-p(x) \leq \delta_{\infty}$ so we conclude

$$
\delta_{\infty} \geq \int_{0}^{t_{0}}\left\|g\left(\pi_{x}(t)\right)\right\|^{2} d t
$$

Now we partition the interval $\left[0, t_{0}\right]$ into $T_{A}$ and $T_{B}$ where $T_{A}=\left\{t: \pi_{x}(t) \in A_{n}\right\}$ and $T_{B}=\{t$ : $\left.\pi_{x}(t) \in B_{n}\right\}$. Denote $t_{A}=\int_{T_{A}} d t$ and $t_{B}=\int_{T_{B}} d t$ as the length of $T_{A}$ and $T_{B}$. Note that $t_{0}=t_{A}+t_{B}$. Using $T_{A}$ and $T_{B}$, we rewrite equation (14) as

$$
\delta_{\infty} \geq \int_{0}^{t_{0}}\left\|g\left(\pi_{x}(t)\right)\right\|^{2} d t=\int_{T_{A}}\left\|g\left(\pi_{x}(t)\right)\right\|^{2} d t+\int_{T_{B}}\left\|g\left(\pi_{x}(t)\right)\right\|^{2} d t .
$$

In the case of $T_{A}$, by the first assertion of Lemma 16, we have a lower bound on the gradient: $\left\|g\left(\pi_{x}(t)\right)\right\| \geq C_{\min } R_{n}$ for some constant $C_{\min }$. Thus,

$$
\int_{T_{A}}\left\|g\left(\pi_{x}(t)\right)\right\|^{2} d t \geq \int_{T_{A}}\left(C_{\min } R_{n}\right)^{2} d t=C_{\min }^{2} R_{n}^{2} t_{A}
$$

To bound $T_{B}$, for simplicity we assume $T_{B}=\left[t_{*}, t_{* *}\right]$ (if $T_{B}$ contains multiple intervals, we can do the same thing for each of them). At the beginning of $T_{B}\left(t=t_{*}\right), \pi_{x}\left(t_{*}\right)$ is on the boundary of the ball $B\left(c, R_{n}\right)$ so $g\left(\pi_{x}\left(t_{*}\right)\right) \geq C_{\min } R_{n}$ by Lemma 16 . For $t \in\left[t_{*}, t_{* *}\right]$, we have

$$
\begin{aligned}
\left\|g\left(\pi_{x}(t)\right)\right\|^{2} & =\left\|g\left(\pi_{x}\left(t_{*}\right)\right)\right\|^{2}+\int_{s=t_{*}}^{s=t}\left(\frac{d\left\|g\left(\pi_{x}(s)\right)\right\|^{2}}{d s}\right) d s \\
& \geq C_{\min }^{2} R_{n}^{2}+\int_{s=t_{*}}^{s=t} 2 \pi_{x}^{\prime}(s)^{T} H\left(\pi_{x}(s)\right) g\left(\pi_{x}(s)\right) d s \\
& =C_{\min }^{2} R_{n}^{2}+2 \int_{s=t_{*}}^{s=t} g^{T}\left(\pi_{x}(s)\right) H\left(\pi_{x}(s)\right) g\left(\pi_{x}(s)\right) d s .
\end{aligned}
$$

Note that in the last equality we use the property of a gradient flow: $\pi_{x}^{\prime}(s)=g\left(\pi_{x}(s)\right)$. Because $\pi_{x}(s) \in B\left(c, R_{n}\right)$ when $s \in\left[t_{*}, t_{* *}\right]$, the second assertion of Lemma 16 implies $\left\|g\left(\pi_{x}(s)\right)\right\| \leq C_{\max } R_{n}$. Let $\|M\|_{*}$ denotes the spectral norm of the matrix $M$. Then

$$
g^{T}\left(\pi_{x}(s)\right) H\left(\pi_{x}(s)\right) g\left(\pi_{x}(s)\right) \geq-\sup _{y}\|H(y)\|_{*} \sup _{y \in B_{n}}\|g(y)\|^{2} \geq-C_{\max }^{\prime} R_{n}^{2},
$$


for some constant $C_{\max }^{\prime}>0$. Note that the spectral norm is bounded because of $(\mathrm{P} 1)$ - the second derivative of $p$ is uniformly bounded. Thus, equation (17) implies

$$
\left\|g\left(\pi_{x}(t)\right)\right\|^{2} \geq C_{\min }^{2} R_{n}^{2}-C_{\max }^{\prime} R_{n}^{2} \int_{s=t_{*}}^{s=t} d s=C_{\min }^{2} R_{n}^{2}-C_{\max }^{\prime} R_{n}^{2}\left(t-t_{*}\right) .
$$

This implies the following bound

$$
\begin{aligned}
\int_{T_{B}}\left\|g\left(\pi_{x}(t)\right)\right\|^{2} d t & \geq \int_{t_{*}}^{t_{* *}}\left(C_{\min }^{2} R_{n}^{2}-C_{\max }^{\prime} R_{n}^{2}\left(t-t_{*}\right)\right) d t \\
& =C_{\min }^{2} R_{n}^{2} t_{B}-0.5 C_{\max }^{\prime} R_{n}^{2} t_{B}^{2} .
\end{aligned}
$$

Putting equations (16) and (18) into equation (15) and using the fact that $t_{0}=t_{A}+t_{B}$, we obtain

$$
\begin{aligned}
\delta_{\infty} & \geq \int_{T_{A}}\left\|g\left(\pi_{x}(t)\right)\right\|^{2} d t+\int_{T_{B}}\left\|g\left(\pi_{x}(t)\right)\right\|^{2} d t \\
& \geq C_{\min }^{2} R_{n}^{2} t_{A}+C_{\min }^{2} R_{n}^{2} t_{B}-0.5 C_{\max }^{\prime} R_{n}^{2} t_{B}^{2} \\
& =C_{\min }^{2} R_{n}^{2} t_{0}-0.5 C_{\max }^{\prime} R_{n}^{2} t_{B}^{2} \\
& \geq C_{\min }^{2} R_{n}^{2} t_{0}-0.5 C_{\max }^{\prime} R_{n}^{2} t_{0}^{2} \\
& \geq C_{\min }^{\prime} R_{n}^{2} t_{0}
\end{aligned}
$$

for some positive constant $C_{\min }^{\prime}$ when $t_{0} \rightarrow 0$ (later we will show that this is true). This implies

$$
t_{0}=O\left(\delta_{\infty} / R_{n}^{2}\right) .
$$

Let $x_{\lambda}=\pi_{x}\left(t_{0}\right) \in L_{\lambda}$ be the point where $\pi_{x}(t)$ intersect $L_{\lambda}$. By Fact $\mathbf{1}$,

$$
\begin{aligned}
& \left\|x_{\lambda}-x\right\| \leq \int_{0}^{t_{0}}\left\|\pi_{x}^{\prime}(t)\right\| d t \\
& \leq \sqrt{\int_{0}^{t_{0}}\left\|\pi_{x}^{\prime}(t)\right\|^{2} d t \int_{0}^{t_{0}} 1^{2} d t} \quad \text { (Cauchy-Schwarz inequality) } \\
& =\sqrt{\int_{0}^{t_{0}}\left\|g\left(\pi_{x}(t)\right)\right\|^{2} d t \int_{0}^{t_{0}} 1^{2} d t} \quad\left(\pi_{x}^{\prime}(t)=g\left(\pi_{x}(t)\right)\right) \\
& \leq \sqrt{\delta_{\infty} t_{0}} \quad \text { (equation (14)) } \\
& =O\left(\delta_{\infty} / R_{n}\right) \quad \text { (equation (19)). }
\end{aligned}
$$

Thus, we conclude that $A_{n} \cap\{y: p(y)<\lambda\} \subset\left(\partial L_{\lambda} \oplus O\left(\delta_{\infty} / R_{n}\right)\right)$. Similarly, we can prove that $A_{n} \cap\{y: p(y)>\lambda\} \subset\left(\partial L_{\lambda} \oplus O\left(\delta_{\infty} / R_{n}\right)\right)$ by considering a gradient descent flow. So we conclude

$$
A_{n} \subset\left(\partial L_{\lambda} \oplus O\left(\delta_{\infty} / R_{n}\right)\right) .
$$

Now using Fact 2 (because $p$ is a Morse function and has bounded 4 times differentiations, $\partial L_{\lambda}$ has a bounded surface area), we conclude

$$
\mu\left(A_{n}\right)=O\left(\delta_{\infty} / R_{n}\right)
$$


when $\delta_{\infty} / R_{n} \rightarrow 0$.

Because $B_{n}=D_{\lambda, \infty} \bigcap B\left(c, R_{n}\right) \subset B\left(c, R_{n}\right)$,

$$
\mu\left(B_{n}\right) \leq \mu\left(B\left(c, R_{n}\right)\right)=O\left(R_{n}^{d}\right) .
$$

Therefore, putting it altogether, we conclude

$$
\mu\left(\widehat{L}_{\lambda} \triangle L_{\lambda}\right) \leq \mu\left(D_{\lambda, \infty}\right)=\mu\left(A_{n}\right)+\mu\left(B_{n}\right) \leq O\left(\delta_{\infty} / R_{n}\right)+O\left(R_{n}^{d}\right) .
$$

Choosing $R_{n}=\delta_{\infty}^{\frac{1}{d+1}}$, we obtain

$$
\mu\left(\widehat{L}_{\lambda} \triangle L_{\lambda}\right) \leq O\left(\delta_{\infty}^{\frac{d}{d+1}}\right)
$$

which is the desired rate. Note that this choice of $R_{n}$ does satisfy the requirement in equation (19) that $t_{0}=\delta_{\infty} / R_{n}^{2}=\delta_{\infty}^{\frac{d-1}{d+1}} \rightarrow 0$ when $d \geq 2$ so we have completed the proof.

Before proving Theorem 4, we first note the following lemma from Chazal et al. (2014).

Lemma 17 (Lemma 16 in Chazal et al. (2014)). Let $p$ be a density with compact support $S$. Assume that $S$ is a d-dimensional compact submanifold of $\mathbb{R}^{d}$ with boundary. Assume $p$ is a Morse function with finitely many, distinct, critical values with corresponding critical points $c_{1}, \cdots, c_{k}$. Also assume that $p$ is twice continuously differentiable on the interior of $S$, continuous and differentiable with non vanishing gradient on the boundary of $S$. Then there exists $\epsilon_{0}>0$ such that for all $0<\epsilon<\epsilon_{0}$ the following is true:

for some positive constant $c$, there exists $\eta \geq c \epsilon$ such that, for any density $q$ with support $S$ satisfying

$$
\|p-q\|_{\infty}<\eta, \sup _{x}\|\nabla p(x)-\nabla q(x)\|_{\max }<\eta, \sup _{x}\|\nabla \nabla p(x)-\nabla \nabla p(x)\|_{\max }<\eta,
$$

$q$ is a Morse function with exactly $k$ critical points $c_{1}^{\prime}, \cdots, c_{k}^{\prime}$ such that after relabeling,

$$
\left\|c_{\ell}-c_{\ell}^{\prime}\right\|<\epsilon
$$

and the number of positive eigenvalues of $\nabla \nabla p\left(c_{\ell}\right)$ and $\nabla \nabla q\left(c_{\ell}^{\prime}\right)$ are the same.

Essentially, Lemma 17 shows that when the density, gradient, and Hessian of two functions are sufficiently close, there exists a unique $1-1$ correspondence between critical points in the two functions. Thus, any critical point of $p$ corresponds to a unique critical point of $q$. In the proof of Theorem 4, we will replace $q$ by the $\operatorname{KDE} \widehat{p}_{n}$.

Proof of TheOREM 4. Recall that $\widehat{\alpha}_{n}(x)=\widehat{P}_{n}\left(\widehat{L}_{\widehat{p}_{n}(x)}\right)$ and $\alpha(x)=P\left(L_{p(x)}\right)$. The main idea for the proof is the following decomposition:

$$
\begin{aligned}
\widehat{\alpha}_{n}(x)-\alpha(x)= & \underbrace{\widehat{P}_{n}\left(\widehat{L}_{\widehat{p}_{n}(x)}\right)-P\left(\widehat{L}_{\widehat{p}_{n}(x)}\right)}_{(\mathrm{A})} \\
& +\underbrace{P(\widehat{L}_{\left.\widehat{p}_{n}(x)\right)-P\left(\widehat{L}_{p(x)}\right)}+\underbrace{P\left(\widehat{L}_{p(x)}\right)-P\left(L_{p(x)}\right)}_{(\mathrm{C})} .}_{(\mathrm{B})}
\end{aligned}
$$


We first bound part (B) and part (C). We will use the results in these two parts to bound the case of part (A).

Part (B). Because

$$
\left|P\left(\widehat{L}_{\widehat{p}_{n}(x)}\right)-P\left(\widehat{L}_{p(x)}\right)\right| \leq P\left(\widehat{L}_{\widehat{p}_{n}(x)} \triangle \widehat{L}_{p(x)}\right),
$$

we first investigate a more general bound on the right hand side. Let $\epsilon>0$ be a small number. For the estimated level sets $\widehat{L}_{\lambda}$ and $\widehat{L}_{\lambda+\epsilon}$, we want to control the quantity $P\left(\widehat{L}_{\lambda} \triangle \widehat{L}_{\lambda+\epsilon}\right)$ when $\epsilon \rightarrow 0$. To obtain the bound in equation (20), we choose $\lambda=p(x)$ and $\epsilon=\widehat{p}_{n}(x)-p(x)$.

An interesting result is that $P\left(\widehat{L}_{\lambda} \triangle \widehat{L}_{\lambda+\epsilon}\right)$ differs if $\lambda$ is a critical value (i.e. density value of a critical point) or not. When $\lambda$ is not a critical value, the gradient $g(x)$ has a nonzero lower bound on $\partial \widehat{L}_{\lambda}$ and we have the local approximation

$$
d\left(x, \widehat{L}_{\lambda+\epsilon}\right)=\frac{\epsilon}{\|g(x)\|}+o(\epsilon)
$$

for each $x \in \partial \widehat{L}_{\lambda}$. Thus, this implies $\widehat{L}_{\lambda+\epsilon} \subset \widehat{L}_{\lambda} \oplus O(\epsilon)$ and the set $\widehat{L}_{\lambda+\epsilon} \backslash \widehat{L}_{\lambda}$ has Lebesgue measure $O(\epsilon)$ because of the normal compatibility property and the fact that $\partial \widehat{L}_{\lambda}$ has finite $d-1$ volume. As a result,

$$
P\left(\widehat{L}_{\lambda} \triangle \widehat{L}_{\lambda+\epsilon}\right)=P\left(\widehat{L}_{\lambda+\epsilon} \backslash \widehat{L}_{\lambda}\right)=O(\epsilon) .
$$

When $\lambda$ coincides with a critical value, we need to split the region $\widehat{L}_{\lambda} \triangle \widehat{L}_{\lambda+\epsilon}$ into two subregions:

$$
\begin{aligned}
& A_{n}=\left(\widehat{L}_{\lambda} \triangle \widehat{L}_{\lambda+\epsilon}\right) \bigcap B^{C}\left(c, R_{\epsilon}\right), \\
& B_{n}=\left(\widehat{L}_{\lambda} \triangle \widehat{L}_{\lambda+\epsilon}\right) \bigcap B\left(c, R_{\epsilon}\right),
\end{aligned}
$$

where $R_{0}$ is a nonzero constant and $R_{\epsilon}$ is a constant converging to 0 when $\epsilon \rightarrow 0$.

This idea is similar to the proof of Theorem 3. Using the same calculation as Part $A_{n}$ in the proof of Theorem 3, we obtain the rate

$$
P\left(A_{n}\right)=O_{P}\left(\frac{\epsilon}{R_{\epsilon}}\right) .
$$

The other part $B_{n}$ controls the area around critical points. This part contributes $P\left(B_{n}\right)=O_{P}\left(\epsilon^{d}\right)$. Thus, putting it all together, we have

$$
\begin{aligned}
P\left(\widehat{L}_{\lambda} \triangle \widehat{L}_{\lambda+\epsilon}\right) & =P\left(A_{n}\right)+P\left(B_{n}\right) \\
& =O_{P}\left(\frac{\epsilon}{R_{\epsilon}}\right)+O_{P}\left(R_{\epsilon}^{d}\right) \\
& =O_{P}\left(\epsilon^{\frac{d}{d+1}}\right)
\end{aligned}
$$

when we choose $R_{\epsilon}=O\left(\epsilon^{\frac{1}{d+1}}\right)$.

Note that the area around the critical point is at rate $\sqrt{\epsilon}$. This is because the density at a critical point behaves quadratically, so a difference in density of $\epsilon$ results in a difference in distance of $\sqrt{\epsilon}$. In addition, the choice $R_{n}=O\left(\epsilon^{\frac{1}{d+1}}\right)$ is obviously slower than $\sqrt{\epsilon}$, so equation (22) is valid.

Substituting equations (21) and (22) into equation (20), we conclude that 
- if the density at $x, p(x)$, is not a critical value of $\widehat{p}$,

$$
\left|P\left(\widehat{L}_{\widehat{p}_{n}(x)}\right)-P\left(\widehat{L}_{p(x)}\right)\right|=O_{P}\left(\left|\widehat{p}_{n}(x)-p(x)\right|\right) ;
$$

- if the density at $x, p(x)$, is a critical value of $\widehat{p}$,

$$
\left|P\left(\widehat{L}_{\widehat{p}_{n}(x)}\right)-P\left(\widehat{L}_{p(x)}\right)\right|=O_{P}\left(\mid \widehat{p}_{n}(x)-p(x)^{\frac{d}{d+1}}\right) .
$$

Finally, for a point $x$, the case where its density $p(x)$ might be a critical value of $\widehat{p}_{n}$ occurs only when

$$
|p(x)-p(c)| \leq\left\|\widehat{p}_{n}-p\right\|_{\infty}+O\left(\left\|\widehat{g}_{n}-g\right\|_{\infty}^{2}\right)=O_{P}\left(\left\|\widehat{p}_{n}-p\right\|_{\infty}\right)
$$

for some critical point $c$ of $p$. To see equation (23), first note for each critical point $c$ of $p$, there exists a critical point $\widehat{c}$ of $\widehat{p}_{n}$ that is close to $c$ due to Lemma 17 (this is because the gradient and Hessian of $\widehat{p}_{n}$ are close to the gradient and Hessian of $p$, respectively). Then by Taylor expansion

$$
p(\widehat{c})-p(c)=(\widehat{c}-c)^{T} \underbrace{g(c)}_{=0}+(\widehat{c}-c)^{T} H(x)(\widehat{c}-c)+O_{P}\left(\|\widehat{c}-c\|^{3}\right)=O_{P}\left(\|\widehat{c}-c\|^{2}\right) .
$$

To bound the rate $\|\widehat{c}-c\|$, note that $\widehat{g}_{n}(\widehat{c})=g(c)=0$ so

$$
\widehat{g}_{n}(c)-g(c)=\widehat{g}_{n}(c)-\widehat{g}_{n}(\widehat{c})=\widehat{H}_{n}(c)(c-\widehat{c})+O_{P}\left(\|\widehat{c}-c\|^{2}\right) .
$$

Multiplying both sides by $\widehat{H}_{n}^{-1}(c)$ from the left and take the norm, we obtain

$$
\begin{aligned}
\|\widehat{c}-c\| & =\widehat{H}_{n}^{-1}(c)\left(\widehat{g}_{n}(c)-g(c)\right)\left(1+o_{P}(1)\right) \\
& =O_{P}\left(\left\|\widehat{g}_{n}(c)-g(c)\right\|\right) \\
& =O_{P}\left(\left\|\widehat{g}_{n}-g\right\|_{\infty}\right) .
\end{aligned}
$$

Moreover, because $\left\|\widehat{g}_{n}-g\right\|_{\infty}^{2}=O\left(h^{4}\right)+O_{P}\left(\frac{\log n}{n h^{d+2}}\right)$ and $\left\|\widehat{p}_{n}-p\right\|_{\infty}=O\left(h^{2}\right)+O_{P}\left(\sqrt{\frac{\log n}{n h^{d}}}\right)$, the requirement on $h: h \rightarrow 0, \frac{\log n}{n h^{d+4}} \rightarrow 0$, implies $\left\|\widehat{g}_{n}-g\right\|_{\infty}^{2}=o_{P}\left(\left\|\widehat{p}_{n}-p\right\|_{\infty}\right)$. Putting it altogether,

$$
\begin{aligned}
\left|\widehat{p}_{n}(\widehat{c})-p(c)\right| & \leq\left|\widehat{p}_{n}(\widehat{c})-p(\widehat{c})\right|+|p(\widehat{c})-p(c)| \\
& \leq\left\|\widehat{p}_{n}-p\right\|_{\infty}+O_{P}\left(\|\widehat{c}-c\|^{2}\right) \\
& =\left\|\widehat{p}_{n}-p\right\|_{\infty}+O_{P}\left(\left\|\widehat{g}_{n}-g\right\|_{\infty}^{2}\right) \\
& =O_{P}\left(\left\|\widehat{p}_{n}-p\right\|_{\infty}\right),
\end{aligned}
$$

which proves equation (23).

Recall that $a_{n}$ is a sequence of $n$ such that $\left\|\widehat{p}_{n}-p\right\|_{\infty}=o\left(a_{n}\right)$. Then the above bound can be rewritten as

$$
\left|P\left(\widehat{L}_{\widehat{p}_{n}(x)}\right)-P\left(\widehat{L}_{p(x)}\right)\right|= \begin{cases}O_{P}\left(\left|\widehat{p}_{n}(x)-p(x)\right|\right) & , \text { if }|p(x)-p(c)|>a_{n} \text { for all } c \in \mathcal{C} . \\ O_{P}\left(\left|\widehat{p}_{n}(x)-p(x)\right|^{\frac{d}{d+1}}\right) & , \text { otherwise. }\end{cases}
$$

This bound is uniform for all $x$ because the sequence $a_{n}$ does not depend on $x$.

Part (C). This part is simply applying Theorem 3, which shows that 
- if the density at $x, p(x)$, is not a critical value of $p$,

$$
\left|P\left(\widehat{L}_{p(x)}\right)-P\left(L_{p(x)}\right)\right|=O_{P}\left(\left\|\widehat{p}_{n}-p\right\|_{\infty}\right) ;
$$

- if the density at $x, p(x)$, is a critical value of $p$,

$$
\left|P\left(\widehat{L}_{p(x)}\right)-P\left(L_{p(x)}\right)\right|=O_{P}\left(\left\|\widehat{p}_{n}-p\right\|_{\infty}^{\frac{d}{d+1}}\right) .
$$

Part (A). Let $\delta_{\infty}=\left\|\widehat{p}_{n}-p\right\|_{\infty}$. It is easy to see that

$$
L_{\widehat{p}_{n}(x)+\delta_{\infty}} \subset \widehat{L}_{\widehat{p}_{n}(x)} \subset L_{\widehat{p}_{n}(x)-\delta_{\infty}} .
$$

This is because any point $z \in L_{\widehat{p}_{n}(x)+\delta_{\infty}}$ satisfies $p(z) \geq \widehat{p}_{n}(x)+\delta_{\infty}$ and thus, $\widehat{p}_{n}(z) \geq p(z)-\delta_{\infty} \geq$ $\widehat{p}_{n}(x)$, which implies $z \in \widehat{L}_{\widehat{p}_{n}(x)}$. The other case can be derived similarly. Equation (24) implies

$$
\begin{gathered}
P\left(L_{\widehat{p}_{n}(x)+\delta_{\infty}}\right) \leq P\left(\widehat{L}_{\widehat{p}_{n}(x)}\right) \leq P\left(L_{\widehat{p}_{n}(x)-\delta_{\infty}}\right) \\
\widehat{P}_{n}\left(L_{\widehat{p}_{n}(x)+\delta_{\infty}}\right) \leq \widehat{P}_{n}\left(\widehat{L}_{\widehat{p}_{n}(x)}\right) \leq \widehat{P}_{n}\left(L_{\widehat{p}_{n}(x)-\delta_{\infty}}\right)
\end{gathered}
$$

Now because the collection of set $\mathcal{L}=\left\{L_{\lambda}: \lambda>0\right\}$ has VC dimension 1, by VC theory (see, e.g., Theorem 2.43 in Wasserman 2006),

$$
\sup _{\lambda}\left|\widehat{P}_{n}\left(L_{\lambda}\right)-P\left(L_{\lambda}\right)\right|=O_{P}\left(\sqrt{\frac{\log n}{n}}\right) .
$$

Therefore,

$$
P\left(L_{\widehat{p}_{n}(x)+\delta_{\infty}}\right)+O_{P}\left(\sqrt{\frac{\log n}{n}}\right) \leq \widehat{P}_{n}\left(\widehat{L}_{\widehat{p}_{n}(x)}\right) \leq P\left(L_{\widehat{p}_{n}(x)-\delta_{\infty}}\right)+O_{P}\left(\sqrt{\frac{\log n}{n}}\right) .
$$

This implies that

$$
\left|\widehat{P}_{n}\left(\widehat{L}_{\widehat{p}_{n}(x)}\right)-P\left(\widehat{L}_{\widehat{p}_{n}(x)}\right)\right| \leq P\left(L_{\widehat{p}_{n}(x)-\delta_{\infty}}\right)-P\left(L_{\widehat{p}_{n}(x)+\delta_{\infty}}\right)+O_{P}\left(\sqrt{\frac{\log n}{n}}\right) .
$$

By a similar derivation as part (B), we will obtain a similar bound as equations (21) and (22)

$$
\begin{aligned}
P\left(L _ { \widehat { p } _ { n } ( x ) - \delta _ { \infty } ) - } P \left(L_{\left.\widehat{p}_{n}(x)+\delta_{\infty}\right)}\right.\right. & \begin{cases}O_{P}\left(\delta_{\infty}\right)+O_{P}\left(\sqrt{\frac{\log n}{n}}\right) & \text {, if }|\widehat{p}(x)-p(c)|>\delta_{\infty} \text { for all } c \in \mathcal{C} . \\
O_{P}\left(\delta_{\infty}^{\frac{d}{d+1}}\right)+O_{P}\left(\sqrt{\frac{\log n}{n}}\right) & , \text { otherwise. }\end{cases}
\end{aligned}
$$

Note that the term $O_{P}\left(\sqrt{\frac{\log n}{n}}\right)$ in the above bound is of a smaller order so we can ignore them. As a result, part (A) contributes

$$
\begin{aligned}
\left|\widehat{P}_{n}\left(\widehat{L}_{\widehat{p}_{n}(x)}\right)-P\left(\widehat{L}_{\widehat{p}_{n}(x)}\right)\right| & \\
& = \begin{cases}O_{P}\left(\left\|\widehat{p}_{n}-p\right\|_{\infty}\right) & \text { if }|\widehat{p}(x)-p(c)|>\delta_{\infty} \text { for all } c \in \mathcal{C} . \\
O_{P}\left(\left\|\widehat{p}_{n}-p\right\|_{\infty}^{\frac{d}{d+1}}\right) & , \text { otherwise. }\end{cases}
\end{aligned}
$$


Thus, putting it all together and using the fact that $\delta_{\infty}=\left\|\widehat{p}_{n}-p\right\|_{\infty}=o_{P}\left(a_{n}\right)$, we conclude that uniformly for all $x$,

$$
\widehat{\alpha}_{n}(x)-\alpha(x)= \begin{cases}O_{P}\left(\left\|\widehat{p}_{n}-p\right\|_{\infty}\right) & , \text { if }|p(x)-p(c)|>a_{n} \text { for all } c \in \mathcal{C}, \\ O_{P}\left(\left\|\widehat{p}_{n}-p\right\|_{\infty}^{\frac{d}{d+1}}\right) & , \text { otherwise }\end{cases}
$$

Proof of Lemma 6. Part 1: pointwise bias. Without loss of generality, we assume $x \in \mathbb{K}_{s}$. We first consider the case $m(x)>0$. In this case, there is a higher-dimensional support $\mathbb{K}_{s+m(x)}$ such that $x \in \overline{\mathbb{K}}_{s+m(x)}$. Thus, for any $r>0$, the ball $B(x, r) \bigcap \mathbb{K}_{s+m(x)} \neq \phi$.

Because the kernel function $K$ is supported on $[0,1]$,

$$
p_{h}(x)=\mathbb{E}\left(\widehat{p}_{n}(x)\right)=\int \frac{1}{h^{d}} K\left(\frac{\|x-y\|}{h}\right) d P(y)=\sum_{\ell=0}^{d} \int_{\mathbb{K}_{\ell}} \frac{1}{h^{d}} K\left(\frac{\|x-y\|}{h}\right) d P(y) .
$$

When $h$ is sufficiently small, $B(x, h) \bigcap \mathbb{K}_{\ell}=\phi$ for any $\ell<s$ so the above expression can be rewritten as

$$
p_{h}(x)=\sum_{\ell=s}^{d} \int_{\mathbb{K}_{\ell}} \frac{1}{h^{d}} K\left(\frac{\|x-y\|}{h}\right) d P(y) .
$$

Now by the definition of $m(x), B(x, r) \bigcap \mathbb{K}_{\ell}=\phi$ for every $\ell>s$ and $\ell<s+m(x)$. Thus, we can again rewrite $p_{h}(x)$ as

$$
\begin{aligned}
p_{h}(x)= & \int_{\mathbb{K}_{s}} \frac{1}{h^{d}} K\left(\frac{\|x-y\|}{h}\right) d P(y) \\
& +\sum_{\ell \geq s+m(x)}^{d} \int_{\mathbb{K}_{\ell}} \frac{1}{h^{d}} K\left(\frac{\|x-y\|}{h}\right) d P(y) \\
= & \underbrace{\int_{\mathbb{K}_{s} \bigcap B(x, h)} \frac{1}{h^{d}} K\left(\frac{\|x-y\|}{h}\right) d P(y)}_{(\mathrm{I})} \\
& +\underbrace{\sum_{\ell \geq s+m(x)}^{d} \int_{\mathbb{K}_{\ell} \cap B(x, h)} \frac{1}{h^{d}} K\left(\frac{\|x-y\|}{h}\right) d P(y)}_{(\mathrm{II})} .
\end{aligned}
$$

Using the generalized density on the $s$-dimensional support, the first term equals

$$
\begin{aligned}
(I) & =\int_{\mathbb{K}_{s} \cap B(x, h)} \frac{1}{h^{d}} K\left(\frac{\|x-y\|}{h}\right) d P(y) \\
& =\int_{\mathbb{K}_{s} \cap B(x, h)} \frac{1}{h^{d}} K\left(\frac{\|x-y\|}{h}\right) \rho(y) d y,
\end{aligned}
$$


where $d y$ is integrating with respect to $s$-dimensional areas (strictly speaking, it should be written as a differential $s$-form but here we write $d y$ for simplicity). Because we assume that $\rho(x)$ is at least three-times bounded differentiable, for any $y \in \mathbb{K}_{s}$ that is close to $x$, we have

$$
\rho(y)=\rho(x)+(y-x)^{T} g_{s}(x)+(y-x)^{T} H_{s}(x)(y-x)+o\left(\|x-y\|^{2}\right),
$$

where $g_{s}$ and $H_{s}$ are the generalized gradient and Hessian matrix on $\overline{\mathbb{K}}_{s}$, respectively (see Section 2.3 of the main paper). By substituting this into equation (26) and using the fact that $K(\|x\|)$ is symmetric and $\overline{\mathbb{K}}_{s}$ is an $s$-dimensional manifold, we obtain

$$
\begin{aligned}
(I)= & \int_{\mathbb{K}_{s} \cap B(x, h)} \frac{1}{h^{d}} K\left(\frac{\|x-y\|}{h}\right) \rho(y) d y \\
= & \int_{x+u h \in \mathbb{K}_{s} \cap B(x, h)} \frac{1}{h^{d}} K(\|u\|) \rho(x) d u \cdot h^{s} \\
& +\int_{x+u h \in \mathbb{K}_{s} \cap B(x, h)} \frac{1}{h^{d}} K(\|u\|) u^{T} H_{s}(x) u d u \cdot h^{s+2}+o\left(\frac{h^{s+2}}{h^{d}}\right) \\
= & \rho(x) \cdot \int_{x+u h \in \mathbb{K}_{s} \cap B(x, h)} \frac{1}{h^{d}} K(\|u\|) d u \cdot h^{s}+O\left(\frac{h^{s+2}}{h^{d}}\right) .
\end{aligned}
$$

Note that again in the above integration, $d u$ is integrating with respect to $s$-dimensional area (again, it is actually a differential $s$-form).

Because $\mathbb{K}_{s}$ has positive reach, $\mathbb{K}_{s}$ around $x$ changes smoothly (if the reach is $\chi$, we can move a ball with radius $\chi$ smoothly over every point in $K_{s}$ ). This further implies that when $\epsilon \rightarrow 0$, the (s-dimensional) area of $\mathbb{K}_{s} \bigcap B(x, \epsilon)$ and the area of $B_{s}(0, \epsilon)$ are similar in the sense that

$$
\frac{\mathrm{Vol}_{s}\left(\mathbb{K}_{s} \bigcap B(x, \epsilon)\right)}{\operatorname{Vol}_{s}\left(B_{s}(0, \epsilon)\right)}=1+O\left(\epsilon^{2}\right)
$$

where $\operatorname{Vol}_{s}(A)$ is the $s$-dimensional volume of the set $A$. Thus, we have

$$
\int_{x+u h \in \mathbb{K}_{s} \cap B(x, h)} K(\|u\|) d u=\int_{B_{s}(0,1)} K(\|u\|) d u\left(1+O\left(h^{2}\right)\right)=\frac{1}{C_{s}^{\dagger}}\left(1+O\left(h^{2}\right)\right) .
$$

Using this and the fact that $\tau(x)=s$, the quantity (I) equals

$$
(I)=\frac{1}{C_{s}^{\dagger}} h^{s-d} \rho(x)+O\left(h^{s-d+2}\right)=\frac{1}{C_{\tau(x)}^{\dagger}} h^{\tau(x)-d} \rho(x)+O\left(h^{\tau(x)-d+2}\right) .
$$

Now we bound the second quantity (II). Because the set $\mathbb{K}_{\ell} \bigcap B(x, h)$ has $\ell$-dimensional volume $O\left(h^{\ell}\right)$,

$$
\int_{\mathbb{K}_{\ell} \cap B(x, h)} \frac{1}{h^{d}} K\left(\frac{\|x-y\|}{h}\right) d P(y) \leq \frac{1}{h^{d}} \int_{\mathbb{K}_{\ell} \cap B(x, h)} K(0) \rho_{\max } d y=O\left(h^{\ell-d}\right) .
$$

In addition, the smallest possible $\ell$ is $\ell=s+m(x)$. Using this and the bound on (I), we get

$$
\begin{aligned}
p_{h}(x) & =\frac{1}{C_{s}^{\dagger}} h^{s-d} \rho(x)+O\left(h^{s-d+2}\right)+O\left(h^{s-d+m(x)}\right) \\
& =\frac{1}{C_{\tau(x)}^{\dagger}} h^{\tau(x)-d} \rho(x)+O\left(h^{\tau(x)-d+2}\right)+O\left(h^{\tau(x)-d+m(x)}\right) .
\end{aligned}
$$


Therefore, by multiplying both sides by $C_{s}^{\dagger} h^{d-\tau(x)}$, we obtain

$$
C_{\tau(x)}^{\dagger} h^{d-\tau(x)} \cdot p_{h}(x)=\rho(x)+O\left(h^{2}\right)+O\left(h^{m(x)}\right),
$$

which proves the first assertion. Note that if $m(x)=0$, we will not have the second term (II), so there is no dimensional bias $O\left(h^{m(x)}\right)$.

Part 2: Failure of uniform convergence of the bias. Without loss of generality, let $(s, \ell)$ be the two lower dimensional supports such that $\overline{\mathbb{K}}_{\ell} \bigcap \mathbb{K}_{s} \neq \phi$ and $s<\ell$.

Let $x \in \overline{\mathbb{K}}_{\ell} \bigcap \mathbb{K}_{s}$ be a point on $\mathbb{K}_{s}$. Then by the first assertion, we have

$$
C_{s}^{\dagger} h^{d-s} \cdot p_{h}(x)=\rho(x)+O\left(h^{2}\right)+O\left(h^{m(x)}\right) .
$$

Now, consider a sequence of points where $h \rightarrow 0:\left\{x_{h} \in \mathbb{K}_{\ell}:\left\|x_{h}-x\right\| \leq h^{2}\right\}$. We can always find such a sequence because $\overline{\mathbb{K}_{\ell}} \bigcap \mathbb{K}_{s} \neq \phi$. For such a sequence, the set $B\left(x_{h}, h\right) \bigcap K_{s}$ converges to the set $B(x, h) \bigcap K_{s}$ in the sense that

$$
\frac{P\left(\left(B\left(x_{h}, h\right) \bigcap K_{s}\right) \triangle\left(B(x, h) \bigcap K_{s}\right)\right)}{P\left(B(x, h) \bigcap K_{s}\right)} \rightarrow 0
$$

when $h \rightarrow 0$. This is because the distance between the centers of the two balls shrinks at rate $h^{2}$ but the radius of the ball shrinks at rate $h$.

Thus, $\frac{p_{h}\left(x_{h}\right)}{p_{h}(x)} \rightarrow 1$ when $h \rightarrow 0$. This implies that $C_{s}^{\dagger} h^{d-s} \cdot p_{h}\left(x_{h}\right) \rightarrow \rho(x)$ so

$$
C_{\tau\left(x_{h}\right)}^{\dagger} h^{d-\tau\left(x_{h}\right)} \cdot p_{h}\left(x_{h}\right)=C_{\ell}^{\dagger} h^{d-\ell} \cdot p_{h}\left(x_{h}\right)=\frac{C_{\ell}^{\dagger}}{C_{s}^{\dagger}} h^{s-\ell} \cdot \underbrace{C_{s}^{\dagger} h^{d-s} \cdot p_{h}\left(x_{h}\right)}_{\rightarrow \rho(x)}
$$

diverges (since $\ell>s$ so $h^{d-\ell}$ diverges). Thus, we do not have uniform convergence for the bias.

Before we proceed to the proof of Theorem 8, we first derive a useful lemma about the variance of the KDE.

Lemma 18 (Pointwise variance). Assume (S, P2, K1-2). Then for $x \in \mathbb{K}(h)$,

$$
\begin{aligned}
\operatorname{Var}\left(\widehat{p}_{n}(x)\right) & =O\left(\frac{1}{n h^{2 d-\tau(x)}}\right), \\
\operatorname{Var}\left(C_{\tau(x)} h^{d-\tau(x)} \cdot \widehat{p}_{n}(x)\right) & =O\left(\frac{1}{n h^{\tau(x)}}\right) .
\end{aligned}
$$

Proof. Without loss of generality, let $x \in \mathbb{K}_{s}(h)$. This implies that $B(x, h) \cap \mathbb{K}_{\ell}=\phi$ for all 
$\ell<s$. By definition,

$$
\begin{aligned}
\operatorname{Var}\left(\widehat{p}_{n}(x)\right) & =\mathbb{E}\left(\widehat{p}_{n}(x)-\mathbb{E}\left(\widehat{p}_{n}(x)\right)\right)^{2} \\
& =\mathbb{E}\left(\frac{1}{n h^{d}} \sum_{i=1}^{n}\left(K\left(\frac{\left\|X_{i}-x\right\|}{h}\right)-\mathbb{E}\left(K\left(\frac{\left\|X_{i}-x\right\|}{h}\right)\right)\right)\right)^{2} \\
& =\frac{1}{n^{2} h^{2 d}} \mathbb{E}\left(\sum_{i=1}^{n}\left(K^{2}\left(\frac{\left\|X_{i}-x\right\|}{h}\right)-n\left(\mathbb{E}^{2}\left(K\left(\frac{\left\|X_{i}-x\right\|}{h}\right)\right)\right)\right)\right) \\
& \leq \frac{1}{n h^{2 d}} \mathbb{E}\left(K^{2}\left(\frac{\left\|X_{1}-x\right\|}{h}\right)\right) \\
& =\frac{1}{n h^{2 d}} \int_{B(x, h)} K^{2}\left(\frac{\|y-x\|}{h}\right) d P(y) \\
& =\frac{1}{n h^{2 d}} \sum_{\ell \leq s} \int_{B(x, h) \cap \mathbb{K}_{\ell}} K^{2}\left(\frac{\|y-x\|}{h}\right) \rho(y) d y \\
& \leq \frac{1}{n h^{2 d}} \sum_{\ell \leq s} \int_{x+u h \in B(x, h) \cap \mathbb{K}_{\ell}} K^{2}(\|u\|) \rho_{\max } d u \cdot h^{\ell} \\
& =O\left(\frac{h^{s}}{n h^{2 d}}\right)=O\left(\frac{1}{n h^{2 d-s}}\right) .
\end{aligned}
$$

Note that $d y$ is integrating with respect to $s$-dimensional area and in the last inequality, we use the transform $y=x+u h$. Because $x+u h$ has to be on $\mathbb{K}_{\ell}$, the change of variable gives $d y=d u \cdot h^{s}$ (the reason why we have $h^{s}$ is because both $d y$ and $d u$ are differential $s$-form). The above derivation is for the case $x \in \mathbb{K}_{s}(h)$ so $\tau(x)=s$. This proves the first assertion and the second assertion follows trivially from the first.

Proof of TheOrem 8. Without loss of generality, we pick a point $x \in \mathbb{K}_{s}(h)$. The difference has the following decomposition:

$$
C_{s}^{\dagger} h^{d-s} \cdot \widehat{p}_{n}(x)-\rho(x)=C_{s}^{\dagger} h^{d-s} \cdot\left(\widehat{p}_{n}(x)-p_{h}(x)\right)+C_{s}^{\dagger} h^{d-s} \cdot p_{h}(x)-\rho(x) .
$$

The former part is the stochastic variation and the latter part is the bias. The bias is uniformly controlled by Lemma 6:

$$
C_{s}^{\dagger} h^{d-s} \cdot p_{h}(x)-\rho(x)=O\left(h^{2} \wedge m_{\min }\right) .
$$

Thus, all we need is to control the stochastic variation.

It is well known that the quantity $\left|\widehat{p}_{n}(x)-p_{h}(x)\right|$ can be written as an empirical process (Einmahl and Mason, 2005; Giné and Guillou, 2002) and to control the supremum of the empirical process $\sup _{x \in \mathbb{K}_{s}} C_{s} h^{d-s} \cdot\left|\widehat{p}_{n}(x)-p_{h}(x)\right|$, we need to uniformly bound the variance. By Lemma 18, the variance is uniformly bounded at rate $O\left(\frac{1}{n h^{s}}\right)$. Therefore, by the assumption (K2) and applying Theorem 2.3 in Giné and Guillou (2002), we have

$$
\sup _{x \in \mathbb{K}_{s}(h)}\left|C_{s}^{\dagger} h^{d-s} \cdot\left(\widehat{p}_{n}(x)-p_{h}(x)\right)\right|=O_{P}\left(\sqrt{\frac{\log n}{n h^{s}}}\right),
$$


which together with the bias term proves the desired result for density estimation.

The case of the gradient and the Hessian can be proved in a similar way as the density estimation case so we ignore the proof. The only difference is that the stochastic part has variance $\frac{1}{n h^{s+2}}$ and $\frac{1}{n h^{s+4}}$. The extra +2 and +4 in the power of $h$ come from taking the derivatives.

Proof of Theorem 9. Recall that

$$
\widehat{\alpha}_{n}(x)=1-\widehat{P}_{n}\left(\left\{y: \widehat{p}_{n}(y) \geq \widehat{p}_{n}(x)\right\}\right) .
$$

Now we consider a modified version

$$
\widetilde{\alpha}_{n}(x)=1-P\left(\left\{y: \widehat{p}_{n}(y) \geq \widehat{p}_{n}(x)\right\}\right)=1-P\left(\widehat{\Omega}_{n}(x)\right),
$$

where $\widehat{\Omega}_{n}(x)=\left\{y: \widehat{p}_{n}(y) \geq \widehat{p}_{n}(x)\right\}=\widehat{L}_{\widehat{p}_{n}(x)}$. The idea of the proof is to bound $\left|\widehat{\alpha}_{n}(x)-\widetilde{\alpha}_{n}(x)\right|$ first and then bound $\left|\widetilde{\alpha}_{n}(x)-\alpha(x)\right|$.

Part 1: Bounding $\left|\widehat{\alpha}_{n}(x)-\widetilde{\alpha}_{n}(x)\right|$. This part can be bounded by a similar derivation as part (A) in the proof of Theorem 4 . Here we just highlight the difference. First, instead of using $L_{\widehat{p}_{n}(x)+\delta_{\infty}} \subset$ $\widehat{\Omega}_{n}(x)=\widehat{L}_{\widehat{p}_{n}(x)} \subset L_{\widehat{p}_{n}(x)-\delta_{\infty}}$, we use

$$
\mathbb{A}_{\widehat{\alpha}_{n}(x)+\delta_{n, h, s}} \subset \widehat{\Omega}_{n}(x)=\widehat{L}_{\widehat{p}_{n}(x)} \subset \mathbb{A}_{\widehat{\alpha}_{n}(x)-\delta_{n, h, s}} \cup \mathbb{K}^{C}(h) .
$$

The first inclusion $\left(\mathbb{A}_{\widehat{\alpha}_{n}(x)+\delta_{n, h, s}} \subset \widehat{L}_{\widehat{p}_{n}}(x)\right)$ in equation $(28)$ can be derived as follows. For any $y \in \mathbb{A}_{\widehat{\alpha}_{n}(x)+\delta_{n, h, s}}$, by its definition $\alpha(y) \geq \widehat{\alpha}_{n}(x)+\delta_{n, h, s}$. Thus,

$$
\widehat{\alpha}_{n}(y) \geq \alpha(y)-\delta_{n, h, s} \geq \widehat{\alpha}_{n}(x) .
$$

Thus, $y \in \widehat{\mathbb{A}}_{\widehat{\alpha}_{n}(x)}=\widehat{L}_{\widehat{p}_{n}(x)}$, which proves the first inclusion. To prove the second inclusion $\left(\widehat{L}_{\widehat{p}_{n}} \subset\right.$ $\left.\mathbb{A}_{\widehat{\alpha}_{n}(x)-\delta_{n, h, s}} \cup \mathbb{K}^{C}(h)\right)$, note that if $y \in \widehat{L}_{\widehat{p}_{n}(x)} \cap \mathbb{K}^{C}(h)$, then this is automatically true so we focus on $y \in \widehat{L}_{\widehat{p}_{n}(x)} \backslash \mathbb{K}(h)$ (namely, $y \in \widehat{L}_{\widehat{p}_{n}}$ and is in good regions). $y \in \widehat{L}_{\widehat{p}_{n}(x)}$ implies $\widehat{p}_{n}(y) \geq \widehat{p}_{n}(x)$, which further implies $\widehat{\alpha}_{n}(y) \geq \widehat{\alpha}_{n}(x)$. Moreover, because $y \in \mathbb{K}^{C}(h)$ (no contribution from a lower dimension manifold), Theorem 9 implies

$$
\alpha(y) \underbrace{\geq}_{y \in \mathbb{K}^{C}(h)} \widehat{\alpha}_{n}(y)-\delta_{n, h, s} \underbrace{\geq}_{y \in \widehat{L}_{\widehat{p} n}(x)} \widehat{\alpha}_{n}(x)-\delta_{n, h, s},
$$

which further implies $y \in \mathbb{A}_{\widehat{\alpha}_{n}(x)-\delta_{n, h, s}}$. This proves the second inclusion and equation (28).

Because the set $\mathcal{A}_{1}=\left\{\mathbb{A}_{\varpi}: \varpi \in[0,1]\right\}$ and $\mathcal{A}_{2}=\left\{\mathbb{A}_{\varpi} \cup \mathbb{K}^{C}(h): \varpi \in[0,1]\right\}$ both have VC dimension 1, again we apply the VC theory (see, e.g., Theorem 2.43 in Wasserman 2006) to bound

$$
\begin{array}{r}
\left|\widehat{P}_{n}\left(\mathbb{A}_{\widehat{\alpha}_{n}(x)+\delta_{n, h, s}}\right)-P\left(\mathbb{A}_{\widehat{\alpha}_{n}(x)+\delta_{n, h, s}}\right)\right|=O_{P}\left(\sqrt{\frac{\log n}{n}}\right) \\
\left|\widehat{P}_{n}\left(\mathbb{A}_{\widehat{\alpha}_{n}(x)-\delta_{n, h, s}} \cup \mathbb{K}^{C}(h)\right)-P\left(\mathbb{A}_{\widehat{\alpha}_{n}(x)-\delta_{n, h, s}} \cup \mathbb{K}^{C}(h)\right)\right|=O_{P}\left(\sqrt{\frac{\log n}{n}}\right) .
\end{array}
$$


Finally, Lemma 19 implies $P\left(\mathbb{K}^{C}(h)\right)=O\left(h^{2} \wedge m_{\min }\right)$ and thus,

$$
\begin{aligned}
P\left(\mathbb{A}_{\widehat{\alpha}_{n}(x)-\delta_{n, h, s}}\right. & \left.\cup \mathbb{K}^{C}(h)\right)-P\left(\mathbb{A}_{\widehat{\alpha}_{n}(x)+\delta_{n, h, s}}\right) \\
& \leq P\left(\mathbb{A}_{\widehat{\alpha}_{n}(x)-\delta_{n, h, s}}\right)-P\left(\mathbb{A}_{\widehat{\alpha}_{n}(x)+\delta_{n, h, s}}\right)+P\left(\mathbb{K}^{C}(h)\right) \\
& =P\left(\mathbb{A}_{\widehat{\alpha}_{n}(x)-\delta_{n, h, s}}\right)-P\left(\mathbb{A}_{\widehat{\alpha}_{n}(x)+\delta_{n, h, s}}\right)+O\left(h^{2} \bigwedge m_{\min }\right) .
\end{aligned}
$$

By definition of the $\alpha$-level set $\mathbb{A}_{\varpi}=\{y: \alpha(y) \geq \varpi\}, P\left(\mathbb{A}_{\varpi}\right)=1-\varpi$ (except for the case that $x \in \mathbb{K}_{0}$ is located at a point mass; but this will be a trivial case that the result holds). Therefore,

$$
P\left(\mathbb{A}_{\widehat{\alpha}_{n}(x)-\delta_{n, h, s}}\right)-P\left(\mathbb{A}_{\widehat{\alpha}_{n}(x)+\delta_{n, h, s}}\right)=2 \delta_{n, h, s} .
$$

Putting all these elements into the part (A) of the proof of Theorem 4, we conclude that

$$
\left|\widehat{P}_{n}\left(\widehat{\Omega}_{n}(x)\right)-P\left(\widehat{\Omega}_{n}(x)\right)\right|=2 \delta_{n, h, s}+O_{P}\left(\sqrt{\frac{\log n}{n}}\right)+O\left(h^{2 \wedge m_{\min }}\right)=O\left(\delta_{n, h, s}\right) .
$$

Part 2: Bounding $\left|\widetilde{\alpha}_{n}(x)-\alpha(x)\right|$. By definition of $\alpha(x)$,

$$
\begin{aligned}
1 & -\alpha(x) \\
& =P(\{y: \alpha(y) \geq \alpha(x)\}) \\
& =P(\{y: \tau(y)<\tau(x)\} \bigcup\{y: \tau(y)=\tau(x), \rho(y) \geq \rho(x)\}) \\
& =P(\{y: \tau(y)<\tau(x)\})+P(\{y: \tau(y)=\tau(x), \rho(y) \geq \rho(x)\}) \\
& =P(\Omega(x))+P(D(x)),
\end{aligned}
$$

where $\Omega(x)=\{y: \tau(y)<\tau(x)\}$ and $D(x)=\{y: \tau(y)=\tau(x), \rho(y) \geq \rho(x)\}$. We define $E(x)=\{y$ : $\tau(y)=\tau(x), \rho(y)<\rho(x)\}$ and $\Phi(x)=\{y: \tau(y)>\tau(x)\}$, and then $\Omega(x), D(x), E(x), \Phi(x)$ form a partition of $\mathbb{K}$.

Using the fact that $\Omega(x), D(x), E(x), \Phi(x)$ is a partition of $\mathbb{K}$, we bound the difference

$$
\begin{aligned}
\left|\alpha(x)-\widetilde{\alpha}_{n}(x)\right|= & P\left(\widehat{\Omega}_{n}(x) \triangle(\Omega(x) \cup D(x))\right) \\
= & P\left((\Omega(x) \cup D(x)) \backslash \widehat{\Omega}_{n}(x)\right)+P\left(\widehat{\Omega}_{n}(x) \backslash(\Omega(x) \cup D(x))\right) \\
= & P\left(\Omega(x) \backslash \widehat{\Omega}_{n}(x)\right) \\
& +P\left(D(x) \backslash \widehat{\Omega}_{n}(x)\right)+P\left(\widehat{\Omega}_{n}(x) \cap(E(x) \cup \Phi(x))\right) \\
= & P\left(\Omega(x) \backslash \widehat{\Omega}_{n}(x)\right)+P\left(D(x) \backslash \widehat{\Omega}_{n}(x)\right) \\
& +P\left(\widehat{\Omega}_{n}(x) \cap E(x)\right)+P\left(\widehat{\Omega}_{n}(x) \cap \Phi(x)\right) \\
\leq & \underbrace{P\left(\left(\Omega(x) \backslash \widehat{\Omega}_{n}(x)\right) \cap \mathbb{K}(h)\right)}_{(\mathrm{I})}+\underbrace{P\left(\left(D(x) \backslash \widehat{\Omega}_{n}(x)\right) \cap \mathbb{K}(h)\right)}_{(\mathrm{II})} \\
+ & \underbrace{P\left(\widehat{\Omega}_{n}(x) \cap E(x) \cap \mathbb{K}(h)\right)}_{(\mathrm{III})}+\underbrace{P\left(\widehat{\Omega}_{n}(x) \cap \Phi(x) \cap \mathbb{K}(h)\right)}_{(\mathrm{V})}+\underbrace{\underbrace{}_{(\mathrm{V})}}_{\left(\mathbb{K}^{C}(h)\right)} .
\end{aligned}
$$


Our approach is to first control (I) and (IV) and then control (II) and (III). Note that Lemma 19 controls the quantity $(\mathrm{V})$ :

$$
(V) \leq O\left(h^{2 \wedge m_{\min }}\right)
$$

Bounding (I) and (IV). Without loss of generality, we consider $x \in \mathbb{K}_{s}(h)$. Given a point $x \in \mathbb{K}_{s}(h)$, the contribution (I) is from a lower dimensional support, say $y \in \mathbb{K}_{\ell}(h)$ with $\ell<s$, such that $\alpha(y)>\alpha(x)$ but $\widehat{p}_{n}(x)<\widehat{p}_{n}(y)$. Namely, we wrongly estimate their ordering. The contribution (IV) is in a similar manner but it is for points in a higher dimensional support. The idea of bound these two components is that we will show that when $h \rightarrow 0, \frac{\log n}{n h^{d+2}} \rightarrow 0$, these two components do not contribute at all with an overwhelming probability (a probability $>1-A_{1} e^{-A_{2} \cdot n h^{d}} \rightarrow 1$ for some $\left.A_{1}, A_{2}>0\right)$.

For any point inside $\mathbb{K}_{s}(h)$, the quantity

$$
\delta_{n, h, s}=\sup _{x \in \mathbb{K}_{s}(h)}\left|C_{s}^{\dagger} h^{d-s} \cdot \widehat{p}_{n}(x)-\rho(x)\right|=O\left(h^{2 \wedge m_{\min }}\right)+O_{P}\left(\sqrt{\frac{\log n}{n h^{s}}}\right) .
$$

Therefore,

$$
\widehat{p}_{n}(x) \leq \frac{\rho(x)+\delta_{n, h, s}}{C_{s}^{\dagger} h^{d-s}}
$$

uniformly for every $x \in \mathbb{K}_{s}(h)$.

Similarly, for any $y \in \mathbb{K}_{\ell}(h)$ with $\ell<s$, we obtain

$$
\widehat{p}_{n}(y) \geq \frac{\rho(y)-\delta_{n, h, \ell}}{C_{\ell}^{\dagger} h^{d-\ell}} .
$$

Therefore,

$$
\sup _{y \in \mathbb{K}_{\ell}(h)} \sup _{x \in \mathbb{K}_{s}(h)} \frac{\widehat{p}_{n}(x)}{\widehat{p}_{n}(y)} \leq h^{s-\ell} \cdot \frac{C_{\ell}^{\dagger}}{C_{s}^{\dagger}} \cdot \frac{\rho_{\max }+\delta_{n, h, s}}{\rho_{\min }-\delta_{n, h, \ell}},
$$

where $\rho_{\max }$ and $\rho_{\min }$ is the maximum and minimum Hausdorff density (across various support) from assumption (P2). Note that if the right-hand-side (33) is less than 1, then $\widehat{p}_{n}(y)>\widehat{p}_{n}(x)$ for every $y \in \mathbb{K}_{\ell}(h)$ and $x \in \mathbb{K}_{s}(h)$. Namely, we will be able to separate points in different dimensional supports if these points are in the good region $\mathbb{K}(h)$ and equation (33) is less than 1.

Thus, when

$$
\max _{\ell<s} h^{s-\ell} \cdot \frac{C_{\ell}^{\dagger}}{C_{s}^{\dagger}} \cdot \frac{\rho_{\max }+\delta_{n, h, s}}{\rho_{\min }-\delta_{n, h, \ell}}<1
$$

we have

$$
\widehat{p}_{n}(y)>\widehat{p}_{n}(x), \quad \forall y \in \mathbb{K}_{\ell}(h), x \in \mathbb{K}_{s}(h), \ell<s .
$$

Because $\Omega(x) \cap \mathbb{K}(h)$ is the regions within $\mathbb{K}(h)$ that is inside a lower dimensional support compared to the point $x$, equation (34) implies

$$
\left(\Omega(x) \backslash \widehat{\Omega}_{n}(x)\right) \cap \mathbb{K}(h)=\emptyset
$$


and $P\left(\left(\Omega(x) \backslash \widehat{\Omega}_{n}(x)\right) \cap \mathbb{K}(h)\right)=0$. A good news is that when $h$ is sufficiently small, equation (34) holds whenever

$$
\max _{\ell \leq s} \delta_{n, h, \ell}<\frac{1}{2} \rho_{\min }
$$

(note that the upper bound $\frac{1}{2} \rho_{\text {min }}$ is just a convenient choice). Since $\delta_{n, h, \ell}$ is just the supremum deviation of the KDE at the $\ell$-dimensional support, by Assumption (K2) and Talagrand's inequality (Giné and Guillou, 2002; Einmahl and Mason, 2005), when $h \rightarrow 0$,

$$
P\left(\delta_{n, h, \ell}<\rho_{\min }\right) \geq 1-A_{1} e^{-A_{2} \cdot n h^{\ell}}
$$

for some constants $A_{1}, A_{2}>0$ (possibly depending on $\ell$ ). Thus,

$$
P\left(\max _{\ell \leq s} \delta_{n, h, \ell}<\frac{1}{2} \rho_{\min }\right) \geq 1-A_{3} e^{-A_{4} \cdot n h^{s}}
$$

for some constants $A_{3}, A_{4}$ (possibly depending on $s$ ). Therefore, when $n h^{s} \rightarrow \infty$ (our assumption on $h$ implies this),

$$
P\left(\left(\Omega(x) \backslash \widehat{\Omega}_{n}(x)\right) \cap \mathbb{K}(h)\right)=0
$$

with a probability greater than or equal to $1-A_{3} e^{-A_{4} \cdot n h^{s}} \rightarrow 1$. Namely, quantity $(\mathrm{I})=0$ with an overwhelming probability. Thus, we can ignore the contribution from (I).

The similar analysis also holds for the case of (IV) since (IV) is also a contribution from wrongly estimating the probability from a different dimensional support. Note that in this case, $(\mathrm{IV})=0$ when $\max _{s \leq \ell} \delta_{n, h, \ell}<\frac{1}{2} \rho_{\text {min }}$, which has a probability

$$
P\left(\max _{s \leq \ell} \delta_{n, h, \ell}<\frac{1}{2} \rho_{\min }\right) \geq 1-A_{5} e^{-A_{6} \cdot n h^{d}},
$$

for some constant $A_{5}, A_{6}>0$. Thus, the requirement on $h$ also implies that such a probability bound converges toward 1 extremely fast. Therefore, the contribution from both (I) and (IV) are 0 with an overwhelming probability so we can ignore them (their contribution will be of the order $O_{P}\left(1-A_{5} e^{-A_{6} \cdot n h^{d}}\right)$, which is much smaller than the other components).

Bounding (II) and (III). We consider cases of (II) and (III) together. Again, we consider $x \in \mathbb{K}_{s}$. Recall that bounds (II) and (III) are the probabilities within the regions

$$
\left(D(x) \backslash \widehat{\Omega}_{n}(x)\right) \cap \mathbb{K}(h), \quad \widehat{\Omega}_{n}(x) \cap E(x) \cap \mathbb{K}(h) .
$$

Now define the region $\Psi(x ; h)=\{y: \tau(y)=\tau(x)\} \cap \mathbb{K}(h)$. Thus, we have

$$
\begin{aligned}
(I I)+(I I I) & \leq P\left(\left(\widehat{\Omega}_{n}(x) \triangle \Omega(x)\right) \cap \Psi(x ; h)\right) \\
& =P\left(\left(\widehat{\Omega}_{n}(x) \cap \Psi(x ; h)\right) \triangle(\Omega(x) \cap \Psi(x ; h))\right) .
\end{aligned}
$$

The event

$$
\begin{array}{r}
\widehat{\Omega}_{n}(x) \cap \Psi(x ; h)=\left\{y \in \mathbb{K}(h): \widehat{p}_{n}(y) \geq \widehat{p}_{n}(x), \tau(y)=\tau(x), x \in \mathbb{K}(h)\right\} \\
=\left\{y \in \mathbb{K}(h): C_{s}^{\dagger} h^{d-s} \cdot \widehat{p}_{n}(y) \geq C_{s}^{\dagger} h^{d-s} \cdot \widehat{p}_{n}(x),\right. \\
\tau(y)=\tau(x)=s, x \in \mathbb{K}(h)\},
\end{array}
$$


which can be viewed as the estimated density upper level set at level $\widehat{p}_{n}(x)$ of the support $\mathbb{K}_{s}(h)$ (because $\tau(x)=s$ ). Similarly, $\Omega(x) \cap \Psi(x ; h)$ is just the upper level set at level $\rho(x)$ of the support $\mathbb{K}_{s}(h)$. Therefore, the difference can be bounded by Theorem 4 :

$$
(I I)+(I I I) \leq \begin{cases}O\left(\delta_{n, h, s}\right), & \text { if } \inf _{c \in \mathcal{C}_{s}}|p(x)-p(c)|>r_{n, h, s} \\ O\left(\left(\delta_{n, h, s}\right)^{\frac{s}{s+1}}\right), & \text { otherwise }\end{cases}
$$

where $r_{n, h, s}$ is a deterministic quantity such that $\delta_{n, h, s}=o_{P}\left(r_{n, h, s}\right)$.

Thus, putting the above bound and equation (31) into equation (30), we have

$$
\begin{aligned}
\left|\alpha(x)-\widetilde{\alpha}_{n}(x)\right| \leq & (I)+(I I)+(I I I)+(I V)+(V) \\
\leq & O\left(h^{\left.2 \bigwedge m_{\min }\right)}\right. \\
& + \begin{cases}O\left(\delta_{n, h, s}\right), & \text { if } \inf _{c \in \mathcal{C}_{s}}|p(x)-p(c)|>r_{n, h, s} \\
O\left(\left(\delta_{n, h, s}\right)^{\frac{s}{s+1}}\right), & \text { otherwise }\end{cases} \\
= & \begin{cases}O\left(\delta_{n, h, s}\right), & \text { if } \inf _{c \in \mathcal{C}_{s}}|p(x)-p(c)|>r_{n, h, s} \\
O\left(\left(\delta_{n, h, s}\right)^{\frac{s}{s+1}}\right), & \text { otherwise }\end{cases}
\end{aligned}
$$

which, along with the fact that $\left|\widehat{\alpha}_{n}(x)-\widetilde{\alpha}_{n}(x)\right|=O\left(\delta_{n, h, s}\right)$, proves the desired result.

Before we move on to the proof of Theorem 10, we first give a lemma that quantifies the size of good regions.

Lemma 19 (Size of good region). Assume (S, P2, K1-2). Define $m_{\min }$ from equation (9) of the main paper and

$$
m_{\min }^{*}=d-\max \left\{s<d: \mathbb{K}_{s} \cap \overline{\mathbb{K}_{d}} \neq \emptyset\right\} .
$$

Let $\mu$ be the Lebesgue measure. Then

$$
\begin{aligned}
& \mu\left(\mathbb{K}^{C}(h)\right)=O\left(h^{m_{\min }^{*}}\right), \\
& P\left(\mathbb{K}^{C}(h)\right)=O\left(h^{m_{\min }}\right) .
\end{aligned}
$$

Proof. Case of the Lebesgue measure. By assumption (S), all supports $\mathbb{K}_{s}$ have Lebesgue measure $\mu\left(\mathbb{K}_{s}\right)=0$ except $\mathbb{K}_{d}$. Thus,

$$
\mu\left(\mathbb{K}^{C}(h)\right)=\mu\left(\mathbb{K}_{d}^{C}(h)\right) .
$$

By the definition of $m_{\text {min }}^{*}$, the quantity $d-m_{\text {min }}^{*}=\max \left\{s<d: \mathbb{K}_{s} \cap \overline{\mathbb{K}_{d}} \neq \emptyset\right\}$ denotes the support with the largest dimension that intersects the closure of $\mathbb{K}_{d}$.

Note that for any two compact sets $A, B$ with dimensions $d(a)$ and $d(b)$ such that $d(b)>d(a)$ and $\bar{A} \cap \bar{B} \neq \phi$, then the $d(b)$-dimensional Lebesgue measure

$$
B \cap(A \oplus r)=O\left(r^{d(b)-d(a)}\right)
$$


when $r \rightarrow 0$. Thus, the set $\mu\left(\mathbb{K}_{d} \cap\left(\mathbb{K}_{d-m_{\min }^{*}} \oplus h\right)\right)$ shrinks at rate $O\left(h^{m_{\text {min }}^{*}}\right)$.

Recall that $\mathbb{K}_{d}(h)=\mathbb{K}_{d} \backslash\left(\bigcup_{\ell<d} \mathbb{K}_{\ell} \oplus h\right)$ and $\mathbb{K}_{d}^{C}(h)=\left(\bigcup_{\ell<d} \mathbb{K}_{\ell} \oplus h\right)$. Because only sets in $\mathbb{K}_{d}$ have nonzero Lebesgue measure, we have

$$
\begin{aligned}
\mu\left(\mathbb{K}_{d}^{C}(h)\right) & =\mu\left(\mathbb{K}_{d}^{C}(h) \cap \mathbb{K}_{d}\right) \\
& =\mu\left(\mathbb{K}_{d} \cap\left(\bigcup_{\ell<d} \mathbb{K}_{\ell} \oplus h\right)\right) \\
& =\mu\left(\mathbb{K}_{d} \cap\left(\bigcup_{\ell \leq d-m_{\min }^{*}} \mathbb{K}_{\ell} \oplus h\right)\right) \\
& \leq \sum_{\ell=0}^{d-m_{\min }^{*}} \mu\left(\mathbb{K}_{d} \cap\left(\mathbb{K}_{\ell} \oplus h\right)\right) \\
& =O\left(h^{m_{\min }^{*}}\right)+o\left(h^{m_{\min }^{*}}\right),
\end{aligned}
$$

which proves the first assertion.

Case of the probability measure. The case of the probability measure is very similar to that of Lebesgue measure, but now we also need to consider lower-dimensional supports because of the singular probability measure.

First, we expand the probability of bad regions by the following:

$$
\begin{aligned}
P\left(\mathbb{K}^{C}(h)\right) & =1-P(\mathbb{K}(h)) \\
& =1-P\left(\bigcup_{\ell \leq d} \mathbb{K}_{\ell}(h)\right) \\
& =1-\sum_{\ell=0}^{d} P\left(\mathbb{K}_{\ell}(h)\right) \\
& =1-\sum_{\ell=0}^{d} P\left(\mathbb{K}_{\ell} \backslash\left(\bigcup_{s<\ell} \mathbb{K}_{s} \oplus h\right)\right) \\
& =\sum_{\ell=0}^{d} P\left(\mathbb{K}_{\ell}\right)-P\left(\mathbb{K}_{\ell} \backslash\left(\bigcup_{s<\ell} \mathbb{K}_{s} \oplus h\right)\right) \\
& =\sum_{\ell=0}^{d} P\left(\mathbb{K}_{\ell} \bigcap\left(\bigcup_{s<\ell} \mathbb{K}_{s} \oplus h\right)\right) .
\end{aligned}
$$

Note that we use the fact that $P(A)-P(A \backslash B)=P(A \cap B)$ in the last equality. We will show that

$$
P\left(\mathbb{K}_{\ell} \bigcap\left(\bigcup_{s<\ell} \mathbb{K}_{s} \oplus h\right)\right)=O\left(h^{m_{\min }}\right) .
$$

for every $\ell$. For simplicity, we define $\mathbb{K}_{\ell}^{C}(h)=\mathbb{K}_{\ell} \bigcap\left(\bigcup_{s<\ell} \mathbb{K}_{s} \oplus h\right)$.

Without loss of generality, we consider the support $\mathbb{K}_{\ell}$ and $\mathbb{K}_{\ell}^{C}(h)$. For simplicity, by the definition of $m_{\text {min }}$, the largest lower-dimensional support that intersects the closure $\overline{\mathbb{K}_{\ell}}$ has a dimension lower 
than or equal to $\mathbb{K}_{\ell-m_{\min }}$. Note that if $\ell<m_{\min }$, it is easy to see that there will be no other lower dimensional support intersecting $\overline{\mathbb{K}_{\ell}}$, so $\mathbb{K}_{\ell}(h)=\mathbb{K}_{\ell}$ and there is nothing to prove. Thus, the set $\mathbb{K}_{\ell}^{C}(h)$ can be rewritten as

$$
\mathbb{K}_{\ell}^{C}(h)=\mathbb{K}_{\ell} \bigcap\left(\bigcup_{s \leq \ell-m_{\min }} \mathbb{K}_{s} \oplus h\right)
$$

By equation (36), the $\ell$-dimensional Lebesgue measure $\mu_{\ell}$ on the set $\mathbb{K}_{\ell}^{C}(h)$ is at rate

$$
\mu_{\ell}\left(\mathbb{K}_{\ell}^{C}(h)\right)=O\left(h^{m_{\min }}\right)+o\left(h^{m_{\min }}\right) .
$$

By assumption (P2), the $\ell$-dimensional Lebesgue measure on $\mathbb{K}_{\ell}$ implies that bound on the probability measure, so we have

$$
P\left(\mathbb{K}_{\ell}^{C}(h)\right)=O\left(h^{m_{\min }}\right) .
$$

Because this works for every $\ell$, by equations (37) and (38), we have

$$
P\left(\mathbb{K}^{C}(h)\right)=O\left(h^{m_{\min }}\right),
$$

which proves the result.

Proof of Theorem 10. We first note that it is easy to see that $m_{\min }^{*} \geq m_{\min }$, so the rate of the integrated error is bounded by that of the probability error. Thus, we only prove the case for the probability error here.

Because $\mathbb{K}_{0}, \cdots, \mathbb{K}_{d}$ form a partition of $\mathbb{K}$, we separately analyze the probability error at each $\mathbb{K}_{\ell}$ and then join them to get the final bound.

For a support $\mathbb{K}_{\ell}$, we partition it into three subregions $A, B$, and $C$, where

$$
\begin{aligned}
& A=\mathbb{K}_{\ell}^{C}(h)=\mathbb{K}_{\ell} \backslash \mathbb{K}_{\ell}(h) \\
& B=\mathbb{K}_{\ell}(h) \cap\left\{x: \min _{c \in \mathcal{C}_{s}}|\rho(x)-\rho(c)| \leq r_{n, h, s}\right\} \\
& C=\mathbb{K}_{\ell}(h) \cap\left\{x: \min _{c \in \mathcal{C}_{s}}|\rho(x)-\rho(c)|>r_{n, h, s}\right\},
\end{aligned}
$$

where $r_{n, h, s}=\frac{h^{2} \wedge m_{\min }+\frac{\log n}{n h^{s}}}{\log n}$ satisfies the requirement $\frac{\delta_{n, h, s}}{r_{n, h, s}}=o_{P}(1)$.

Case A. By Lemma 19, $P(A)=O\left(h^{m_{\min }}\right)$ and $|\widehat{\alpha}(x)-\alpha(x)| \leq 1$. Thus,

$$
\int_{A}\left|\widehat{\alpha}_{n}(x)-\alpha(x)\right| d P(x)=O\left(h^{m_{\min }}\right) .
$$

Case B. For set $B$, note that the generalized density $\rho(x)$ behaves quadratically around critical points. So a difference in density level at rate $\delta$ results in a change in the difference in distance at rate $\sqrt{\delta}$. Thus, the $\ell$-dimensional Lebesgue measure $\mu_{\ell}(B)=O\left(\sqrt{r_{n, h, s}}\right)$, which by assumption (P2) implies $P(B)=O\left(\sqrt{r_{n, h, s}}\right)$. By Theorem $9,\left|\widehat{\alpha}_{n}(x)-\alpha(x)\right|=\delta_{n, h, s}^{\frac{s}{s+1}}$ uniformly for all $x \in B$. Thus, the error is

$$
\int_{B}\left|\widehat{\alpha}_{n}(x)-\alpha(x)\right| d P(x)=O\left(\sqrt{r_{n, h, s}} \cdot \delta_{n, h, s}^{\frac{s}{s+1}}\right) .
$$


Case C. For points in this region, directly applying Theorem 9 yields

$$
\int_{C}\left|\widehat{\alpha}_{n}(x)-\alpha(x)\right| d P(x)=O\left(\delta_{n, h, s}\right) .
$$

By adding up equations (40), (41), and (42) and using the fact that $\sqrt{r_{n, h, s}} \cdot \delta_{n, h, s}^{\frac{s}{s+1}}=o\left(\delta_{n, h, s}\right)$ and $O\left(h^{m_{\min }}\right)$ are part of $\delta_{n, h, s}$, we obtain

$$
\int_{\mathbb{K}_{\ell}}\left|\widehat{\alpha}_{n}(x)-\alpha(x)\right| d P(x)=O\left(\delta_{n, h, s}\right) .
$$

This works for every $\mathbb{K}_{\ell}$, which proves the desired bound.

Proof of Lemma 11. First assertion. By assumption (B) and (P2), the first assertion is trivially true because when we move down the level $\alpha$, the only situation that creates a new connected component is when $\alpha$ passes through the $\alpha$-level of a local mode.

Second assertion. For level sets, there are only two situations where a change in topology may occur: creation of a new connected component, and merging of two (or more) connected components. By the first assertion, we only need to focus on the merging case.

Assume $\varpi \in \mathcal{A}$ be a level where only merging of connected components occurs. In addition, recall that $\xi(\varpi)$ is the integer such that $\mathbb{K}_{s} \subset \mathbb{A}_{\varpi}$ for all $s \leq \xi(\alpha)$ and $\mathbb{K}_{\xi(\varpi)+1} \not \subset \mathbb{A}_{\varpi}$. For a sufficiently small $\epsilon$, the difference between $\mathbb{A}_{\varpi}$ and $\mathbb{A}_{\varpi+\epsilon}$ is in $\mathbb{K}_{\xi(\varpi)+1}$. Thus, when we move $\epsilon$ down, only the connected components in $\mathbb{K}_{\xi(\alpha)+1}$ expand. When merging occurs, there are only two cases: two connected components in $\mathbb{K}_{\xi(\varpi)+1}$ meet each other or a connected component in $\mathbb{K}_{\xi(\varpi)+1}$ hits a lower-dimensional support. Note that there is no higher-dimensional supports in $\mathbb{A}_{\varpi}$ because their ordering is less than any point in $\mathbb{K}_{\xi(\varpi)}$. The first case corresponds to a saddle point, which is an element in $\mathcal{C}$. The second case corresponds to a DCP. Thus, $\varpi$ must be either an $\alpha$-level of a critical point or a DCP.

Proof of Lemma 12. Critical points. Without loss of generality, let $c$ be a critical point of $\rho(x)$ on $\mathbb{K}_{s}$. Recall that $p_{h}=\mathbb{E}\left(\widehat{p}_{n}\right)$ is the smoothed density function and $g_{h}=\nabla p_{h}$ and $H_{h}=\nabla \nabla p_{h}$ are the corresponding gradient and Hessian.

The idea of the proof is to argue that when $\delta_{n, h, s}^{(2)} \rightarrow 0$, we can always find a critical point $\widehat{c}$ of $\widehat{p}_{n}$ and a critical point $c_{h}$ of $p_{h}$ such that

$$
\left\|\widehat{c}-c_{h}\right\|=O_{P}\left(\frac{1}{n h^{s+2}}\right), \quad\left\|c_{h}-c\right\|=O(h) .
$$

Then we use the triangle inequality to bound $\|\widehat{c}-c\|$.

Before we go to the details, we first define a useful set:

$$
\widetilde{\mathbb{K}}_{s}(h)=\left\{x: x \in \mathbb{K}_{s} \oplus h, x \notin \mathbb{K}_{\ell} \oplus h, \ell<s\right\}=\left(\mathbb{K}_{s} \oplus h\right) \backslash \bigcup_{\ell<s}\left(\mathbb{K}_{\ell} \oplus h\right) .
$$

The set $\widetilde{\mathbb{K}}_{s}$ is the regions around $\mathbb{K}_{s}$ but away from a lower dimensional support $\left(\widetilde{\mathbb{K}}_{s}\right.$ is thicker than the 'good region' $\overline{\mathbb{K}}_{s} ; \overline{\mathbb{K}}_{s}$ does not include regions around $\mathbb{K}_{s}$ ). Assumption (C) implies that 
$c \in \widetilde{\mathbb{K}}_{s}(h)$ when $h$ is sufficiently small. Note that any point $x \in \widetilde{\mathbb{K}}_{s}$ has a distance to $\mathbb{K}_{s}$ less than $h$, i.e., $\sup _{x \in \widetilde{\mathbb{K}}_{s}} d\left(x, \mathbb{K}_{s}\right) \leq h$. Using the same bias analysis of $p_{h}$ in Lemma 6 , one can prove that when $h \rightarrow 0$

$$
\sup _{x \in \widetilde{\mathbb{K}}_{s}(h)} h^{d-s} \max \left\{p_{h}(x),\left\|g_{h}(x)\right\|_{\max },\left\|H_{h}(x)\right\|_{\max }\right\} \leq U_{0}
$$

for some fix constant $U_{0}$. An intuitive explanation for Equation (44) is that within the set $\widetilde{\mathbb{K}}_{s}(h)$, the support $\mathbb{K}_{s}$ is the dominating structure (lowest dimensional support) so the smoothed version of these quantities will be diverging at the rate $O\left(h^{s-d}\right)$. Rescaling them by $O\left(h^{d-s}\right)$ will uniformly bound all of them (this is the same idea as Lemma 6).

In addition to equation (44), there is another useful fact about the region $\widetilde{\mathbb{K}}_{s}(h)$ : when $\frac{n h^{s+4}}{\log n} \rightarrow \infty$ and $h \rightarrow 0$,

$$
\begin{aligned}
\sup _{x \in \widetilde{\mathbb{K}}_{s}(h)} h^{d-s}\left|\widehat{p}_{n}(x)-p_{h}(x)\right| & =O_{P}\left(\sqrt{\frac{\log n}{n h^{s}}}\right) \\
\sup _{x \in \widetilde{\mathbb{K}}_{s}(h)} h^{d-s}\left\|\widehat{g}_{n}(x)-g_{h}(x)\right\|_{\max } & =O_{P}\left(\sqrt{\frac{\log n}{n h^{s+2}}}\right) \\
\sup _{x \in \widetilde{\mathbb{K}}_{s}(h)} h^{d-s}\left\|\widehat{H}_{n}(x)-H_{h}(x)\right\|_{\max } & =O_{P}\left(\sqrt{\frac{\log n}{n h^{s+4}}}\right) .
\end{aligned}
$$

The proof of these quantity follows the same way as the proof of Lemma 18 and Theorem 8 . Moreover, the $\sqrt{\log n}$ factor will disappear in equation (45) if the bound is taken for a given point (or a fixed sequence of points).

We first show that $\left\|c_{h}-c\right\|=O(h)$. When $h \rightarrow 0$, we can always find a critical point $c_{h}$ of $p_{h}$ such that $c_{h} \rightarrow c$ (because $p_{h}$ is essentially a smoothed version of the singular measure). Note that such a point $c_{h}$ serves as an 'approximation' of $c$ in the function $p_{h}$. Moreover, $d\left(c_{h}, \mathbb{K}_{s}\right)<h$ because regions outside $h$ distance of $\mathbb{K}_{s}$ will not be affected by $\mathbb{K}_{s}$. For any point $x \in \mathbb{K}$, let $\pi_{s}(x) \in \mathbb{K}_{s}$ be the projection from $x$ onto $\mathbb{K}_{s}$. Because $\mathbb{K}_{s}$ has positive reach, the projection $\pi_{s}\left(c_{h}\right)$ will be unique when $h$ is less than the reach of $\mathbb{K}_{s}$. Note that the fact that $d\left(c_{h}, \mathbb{K}_{s}\right)<h$ implies $d\left(c_{h}, \pi_{s}\left(c_{h}\right)\right)=d\left(c_{h}, \mathbb{K}_{s}\right)<h$. By assumption $(\mathrm{C})$, there is a fixed distance between $c$ and any lower dimensional support so $\pi_{s}\left(c_{h}\right) \in \mathbb{K}_{s}(h)$ when $h$ is sufficiently small (namely, the projected point will be in the good region).

Because $d\left(c_{h}, \pi_{s}\left(c_{h}\right)\right)<h$ and the (scaled) Hessian $C_{s}^{\dagger} h^{d-s} H_{h}$ is uniformly bounded within $\widetilde{K}_{s}(h)$ (equation (44)), Taylor theory implies

$$
C_{s}^{\dagger} h^{d-s}\|\underbrace{g_{h}\left(c_{h}\right)}_{=0}-g_{h}\left(\pi_{s}\left(c_{h}\right)\right)\|_{\max }=O\left(\left\|c_{h}-\pi_{s}\left(c_{h}\right)\right\|\right)=O(h) .
$$

Note that $g_{h}(x)=\nabla p_{h}(x)$ so $\left\|g_{h}(x)\right\|_{\max } \geq\left\|\nabla_{T_{s}(x)} p_{h}(x)\right\|_{\max }$ (recall that $\nabla_{T_{s}(x)}$ is the gradient with respect to the tangent space so it has fewer degree of freedom than $\nabla$ ). Thus,

$$
\begin{aligned}
C_{s}^{\dagger} h^{d-s}\left\|g_{h}\left(\pi_{s}\left(c_{h}\right)\right)\right\|_{\max } & =O(h) \\
& \geq\left\|C_{s}^{\dagger} h^{d-s} \nabla_{T_{s}\left(\pi_{s}\left(c_{h}\right)\right)} g_{h}\left(\pi_{s}\left(c_{h}\right)\right)\right\|_{\max } \\
& =\left\|\nabla_{T_{s}\left(\pi_{s}\left(c_{h}\right)\right)} \rho\left(\pi_{s}\left(c_{h}\right)\right)\right\|_{\max }+O\left(h^{2} \wedge m_{\min }\right) .
\end{aligned}
$$


Note that the last equality is from Theorem 8 . Because $m_{\min } \geq 1$, the above inequality further implies

$$
\left\|\nabla_{T_{s}\left(\pi_{s}\left(c_{h}\right)\right)} \rho\left(\pi_{s}\left(c_{h}\right)\right)\right\|_{\max }=O(h) .
$$

When $h \rightarrow 0$, region on $\mathbb{K}_{s}$ with a low gradient of $\rho(x)$ must be around a critical point since $\rho$ on $\mathbb{K}_{s}$ is a Morse function (assumption (P2)). Because the eigenvalues of the Hessian of $\rho(x)$ at $c$ is uniformly bounded away from 0 (an natural outcome of a Morse function), the gradient changes linearly around $c$. As a result, equation (46) implies $\left\|\pi_{s}\left(c_{h}\right)-c\right\|=O(h)$. This, together with the fact that $\left\|\pi_{s}\left(c_{h}\right)-c_{h}\right\|=O(h)$, further implies that $\left\|c_{h}-c\right\|=O(h)$.

To prove that $\left\|\widehat{c}-c_{h}\right\|=O_{P}\left(\sqrt{\frac{1}{n h^{s+2}}}\right)$, by equation (45) the gradient of any point $x \in \widetilde{\mathbb{K}}_{s}(h)$ is

$$
h^{d-s}\left(\widehat{g}_{n}(x)-g_{h}(x)\right)=O_{P}\left(\sqrt{\frac{1}{n h^{s+2}}}\right) .
$$

Plugging-in $x=c_{h}$ into the above equality and using equation (45), we obtain

$$
\begin{aligned}
h^{d-s}(\widehat{g}_{n}\left(c_{h}\right)-\underbrace{g_{h}\left(c_{h}\right)}_{=0}) & =O_{P}\left(\sqrt{\frac{1}{n h^{s+2}}}\right) \\
& =h^{d-s}(\widehat{g}_{n}\left(c_{h}\right)-\underbrace{\widehat{g}_{n}(\widehat{c})}_{=0}) \\
& =h^{d-s} \widehat{H}_{h}\left(c_{h}\right)\left(c_{h}-\widehat{c}\right)+O_{P}\left(\left\|c_{h}-\widehat{c}\right\|^{2}\right) \\
& =h^{d-s} \underbrace{\left(H_{h}\left(c_{h}\right)+O_{P}\left(\sqrt{\frac{\log n}{n h^{s+4}}}\right)\right)}_{\text {by }(45)}\left(c_{h}-\widehat{c}\right)+O_{P}\left(\left\|c_{h}-\widehat{c}\right\|^{2}\right) \\
& =h^{d-s} H_{h}\left(c_{h}\right)\left(c_{h}-\widehat{c}\right)+o_{P}\left(\left\|c_{h}-\widehat{c}\right\|\right) .
\end{aligned}
$$

Since all eigenvalues of $h^{d-s} H_{h}\left(c_{h}\right)$ are uniformly bounded away from 0 when $h \rightarrow 0$ (this follows from the fact that $\rho(x)$ is a Morse function and $\left.K^{(2)}(0)<0\right)$, its inverse exists so $\left\|c_{h}-\widehat{c}\right\|=$ $O_{P}\left(\sqrt{\frac{1}{n h^{s+2}}}\right)$. Thus, putting it altogether we obtain $\|\widehat{c}-c\| \leq\left\|c_{h}-\widehat{c}\right\|+\left\|c_{h}-c\right\|=O(h)+$ $O_{P}\left(\sqrt{\frac{1}{n h^{s+2}}}\right)$, which proves the stability of critical points.

Critical levels. To derive the rate of $\left|\widehat{\alpha}_{n}(\widehat{c})-\alpha(c)\right|$, we decompose it into

$$
\left|\widehat{\alpha}_{n}(\widehat{c})-\alpha(c)\right| \leq\left|\widehat{\alpha}_{n}(\widehat{c})-\widehat{\alpha}_{n}(c)\right|+\left|\widehat{\alpha}_{n}(c)-\alpha(c)\right| \text {. }
$$

Because the KDE around $\widehat{c}$ behaves quadratically, i.e., for any $\mu \in \mathbb{R}^{d}$ such that $\|\mu\|$ is small, $\widehat{p}_{n}(\widehat{c}+\mu)-\widehat{p}_{n}(\widehat{c})=O_{P}\left(\left\|\mu^{2}\right\|\right)$ and thus

$$
\left|\widehat{\alpha}_{n}(\widehat{c})-\widehat{\alpha}_{n}(c)\right|=O\left(\|\widehat{c}-c\|^{2}\right)=O\left(h^{2}\right)+O_{P}\left(\frac{1}{n h^{s+2}}\right)
$$


which is dominated by the second term $\left|\widehat{\alpha}_{n}(c)-\alpha(c)\right|=O\left(\delta_{n, h, s}^{\frac{s}{s+1}}\right)$ (by Theorem 9). Thus, the convergence rate is $\left|\widehat{\alpha}_{n}(\widehat{c})-\alpha(c)\right|=O\left(\delta_{n, h, s}^{\frac{s}{s+1}}\right)$.

Eigenvalues. For the eigenvalues, it is easy to see that when we are moving away from $c$ along a direction that is normal to $\mathbb{K}_{s}$, the estimated density is going down. Thus, these $(d-s)$ directions must have negative eigenvalues (dimension of the normal subspace is $d-s$ ). In addition, the original generalized Hessian matrix at $c$ has $n(c)$ negative eigenvalues. So the total number of negative eigenvalues of the Hessian matrix of $\widehat{p}_{n}$ at $c$ is $n(s)+d-s$. Because $\widehat{c}$ is converging to $c$, the sign of negative eigenvalues also converges, which proves the lemma.

Proof of Lemma 13. First assertion: Location of DCPs. By Theorem 8, the scaled KDE is uniformly consistent in both density estimation and gradient estimation in the good region $\mathbb{K}(h)$.

The estimated DCPs are points satisfying $\nabla \widehat{p}_{n}(x)=0$. Therefore, when $\delta_{n, h, d}^{(2)} \stackrel{P}{\rightarrow} 0$, the only areas in $\mathbb{K}(h)$ such that $\nabla \hat{p}_{n}(x)=0$ will be regions where $\nabla_{T_{\tau(x)}(x)} \rho(x)$ is small. This can only be the regions around the generalized critical points. As a result, we cannot have any DCPs within $\mathbb{K}(h)$ when $\delta_{n, h, d}^{(2)} \stackrel{P}{\rightarrow} 0$.

Second assertion: Number of estimated critical points. This follows directly from Lemma 14 and Assumption (A) and from the fact that $\delta_{n, h, d}^{(2)} \stackrel{P}{\rightarrow} 0$.

Third assertion: No local modes. By assumption (B), the only case where the creation of a connected component occurs is a (generalized) local mode. These population local modes correspond to elements in $\widehat{\mathcal{C}}$. Thus, any estimated local mode in $\widehat{\mathcal{D}}$ does not have a population target so they are away from the population local modes and by first assertion, it has to be in the bad region $\mathbb{K}^{C}(h)$. It is easy to see that we cannot have any estimated local mode under such a constraint when we have the gradient and Hessian consistency of the scaled $\operatorname{KDE}\left(\delta_{n, h, d}^{(2)} \stackrel{P}{\rightarrow} 0\right)$.

Proof of Lemma 14. Let $c$ be a DCP and $\varpi_{0}(c)$ be the corresponding $\alpha$-level of merging. By assumption (A), $c$ is a unique point for $\alpha_{0}(c) \in \mathcal{D}$ and for any sufficiently small $\epsilon$, there is a unique connected component $C_{\epsilon} \in \mathbb{A}_{\varpi_{0}(c)+\epsilon}$ and a support $\mathbb{K}_{\ell}$ with $\ell \leq \xi\left(\varpi_{0}(c)\right)$ such that $x \in \mathbb{K}_{\ell}, c \notin C_{\epsilon}$ and $d\left(c, C_{\epsilon}\right) \rightarrow 0$ when $\epsilon \rightarrow 0$.

The idea of the proof is to find $\widehat{\varpi}^{+}$and $\widehat{\varpi}^{-}$such that the merging has not yet happened in the set $\mathbb{A}_{\widehat{\varpi}}+$ but the merging has happened in the set $\mathbb{A}_{\widehat{\varpi}^{-}}$. Then we know the actual value $\widehat{\varpi}_{n}(\widehat{c})$ lies within the interval $\left[\mathbb{A}_{\widehat{\varpi}^{-}}, \mathbb{A}_{\widehat{\varpi}^{+}}\right]$.

Case: Lower bound. By Theorem 9, any point $y \in K(h)$ satisfies $\left|\widehat{\alpha}_{n}(y)-\alpha(y)\right|=\delta_{n, h, \tau(y)}$. Thus, for any $C_{\epsilon}$ defined as the connected component within $\mathbb{A}_{\varpi_{0}(x)+\epsilon}$ that is about to merge with $\mathbb{K}_{\ell}$,

$$
\inf _{y \in C_{\epsilon}} \widehat{\alpha}_{n}(y)>\varpi_{0}(x)-\delta_{n, h, \xi\left(\varpi_{0}(c)\right)+1}
$$

This is because $\inf _{y \in C_{\epsilon}} \alpha(y) \geq \varpi_{0}(c)$ by definition and for $C_{\epsilon} \cap \mathbb{K}(h)$, we have the uniform bound from Theorem 9. For $y \in C_{\epsilon} \backslash \mathbb{K}(h)$, the estimated $\widehat{\alpha}_{n}(y)$ will be influenced by $\mathbb{K}_{\ell}$, which is a lower dimensional support. So the estimated $\alpha$ value will be more than $\varpi_{0}(c)-\delta_{n, h, \xi\left(\varpi_{0}(c)\right)+1}$. Thus, we can pick the lower bound $\mathbb{A}_{\widehat{a}^{-}}=\varpi_{0}(c)-\delta_{n, h, \xi\left(\varpi_{0}(c)\right)+1}$.

Case: Upper bound. To prove the upper bound, the idea is very simple. We show that when the $\alpha$-level is high enough, $\widehat{\mathbb{A}}_{\varpi^{\prime}}$ will not contain the set $\widetilde{C}_{\ell} \oplus h$ where $c \in \widetilde{C}_{\ell}$ and $\widetilde{C}_{\ell}$ is a connected 
component of $\mathbb{A}_{\varpi_{0}}$.

Let $\widetilde{C}_{\ell}$ be defined as above. Because $\widetilde{C}_{\ell}$ is a subset of $\bigcup_{s \leq \ell} \mathbb{K}_{s}$, the set $C_{\epsilon} \cap\left(\widetilde{C}_{\ell} \oplus h\right)$ is always within the bad region $\mathbb{K}^{C}(h)$. Thus, $P\left(C_{\epsilon} \cap\left(\widetilde{C}_{\ell} \oplus h\right)\right) \leq P\left(\mathbb{K}^{C}(h)\right)=O\left(m^{2} \wedge m_{\min }\right)$ by Lemma 19 .

Now we consider the boundary $\partial\left(\widetilde{C}_{\ell} \oplus h\right)=\left\{x: \bar{d}\left(x, \widetilde{C}_{\ell}\right)=h\right\}$. Because $P\left(C_{\epsilon} \cap\left(\widetilde{C}_{\ell} \oplus h\right)\right) \leq$ $O\left(m^{2} \wedge m_{\min }\right)$

$$
\sup _{x \in \partial\left(\widetilde{C}_{\ell} \oplus h\right)} \alpha(x) \leq \varpi_{0}(c)+O\left(m^{2} \wedge m_{\min }\right) .
$$

Moreover, outside the boundary $\partial\left(\widetilde{C}_{\ell} \oplus h\right)$ we can apply Theorem 9 to bound $\widehat{\alpha}_{n}(x)-\alpha(x)$. Thus,

$$
\sup _{x \in \partial\left(\widetilde{C}_{\ell} \oplus h\right)} \widehat{\alpha}_{n}(x) \leq \varpi_{0}(c)+O\left(m^{2} \wedge m_{\min }\right)+\delta_{n, h, \xi\left(\varpi_{0}(c)\right)+1}
$$

This suggests that $\mathbb{A}_{\widehat{\varpi}^{+}}=a_{0}+O\left(m^{2} \wedge m_{\min }\right)+\delta_{n, h, \xi\left(\varpi_{0}(c)\right)+1}=a_{0}+\delta_{n, h, \xi\left(\varpi_{0}(c)\right)+1}$ because $O\left(m^{2} \wedge m_{\min }\right)$ is part of the term $\delta_{n, h, \xi\left(\varpi_{0}(c)\right)+1}$.

Thus, the quantity $\widehat{\alpha}_{n}(\widehat{c})$ must lie within $a_{0} \pm \delta_{n, h, \xi\left(\varpi_{0}(c)\right)+1}$, which is the desired bound. Because there is a topological change in the upper level set for $\widehat{p}_{n}$ at such $\widehat{\alpha}_{n}(\widehat{c}), \widehat{c}$ must be a critical point of $\widehat{p}_{n}$. This completes the proof.

Proof of Theorem 15. The main idea is to show that there exists a constant $a_{0}>0$ such that

$$
\max _{j=0,1,2} \max _{s=0, \cdots, d}\left|\delta_{n, h, s}^{(j)}\right|<a_{0} \Longrightarrow T_{\widehat{\alpha}_{n}} \stackrel{T}{\approx} T_{\alpha}
$$

Note that $\delta_{n, h, s}^{(0)}=\delta_{n, h, s}$.

By assumption (A), there exists a constant $a_{1}=\min \left\{\left|\alpha_{1}-\alpha_{2}\right|: \alpha_{1}, \alpha_{2} \in \mathcal{A}, \alpha_{1} \neq \alpha_{2}\right\}>0$. Without loss of generality, let the elements in $\mathcal{A}$ be $\alpha_{1}>\alpha_{2}>\cdots>\alpha_{m}$ (assume $\mathcal{A}$ has $m$ elements) and $c\left(\alpha_{j}\right)$ be the corresponding critical point or DCP for level $\alpha_{j}$. By Lemma 12 and 14, we can find a sequence of points $\widehat{c}_{1}, \cdots, \widehat{c}_{m}$ such that each $\widehat{c}_{j}$ is the estimator to $c\left(\alpha_{j}\right)$. Again, by Lemma 12 and 14, when

$$
\max _{s=0, \cdots, d}\left|\delta_{n, h, s}^{(0)}\right|<\frac{a_{1}}{2}
$$

we have

$$
\widehat{\alpha}_{n}\left(\widehat{c}_{1}\right)>\cdots>\widehat{\alpha}_{n}\left(\widehat{c}_{m}\right) .
$$

Namely, the ordering will be the same when $\max _{s=0, \cdots, d}\left|\delta_{n, h, s}^{(0)}\right|$ is sufficiently small. Thus, we need to prove that (1) there is no other connected component of $\widehat{\alpha}_{n}$ and (2) there is no other merging point to get the topological equivalent.

By Lemma 13, there will be no estimated local mode in $\widehat{\mathcal{D}}$ so there will be no extra connected components. In the proof of Lemma 14, we showed that each estimator of a DCP corresponds to a merging in the estimated level sets, and similarly, if connected components are merged at a saddle point or a local minimum, the corresponding estimator will be a merging point. To use the conclusion of Lemma 12, we need uniform consistency in both gradient and Hessian estimation. Namely, we need $\delta_{n, h, s}^{(1)}, \delta_{n, h, s}^{(2)}$ to be sufficiently small. The gradient consistency regularizes the positions of estimators 
of generalized critical points and the bound on the Hessian matrix guarantees that the eigenvalues retain the same sign. Thus, to apply Lemma 12, there is some $a_{2}>0$ such that the conclusion of Lemma 12 holds whenever

$$
\max _{j=1,2} \max _{s=0, \cdots, d}\left|\delta_{n, h, s}^{(j)}\right|<a_{2}
$$

To use Lemma 14, we need $\delta_{n, h, s}^{(1)}$ to be sufficiently small. This comes from Theorem 9.

Combining equations (48) and (49), we obtain equation (47). For the quantity $\delta_{n, h, s}^{j}$, the slowest rate occurs at $j=2$ and $s=d$. Thus, there are some constants $c_{1}, c_{2}>0$ such that

$$
\max _{j=0,1,2} \max _{s=0, \cdots, d}\left|\delta_{n, h, s}^{(j)}\right|<c_{1} h^{2} \wedge m(x)+c_{2} Z_{d}
$$

where $Z_{d}=O_{P}\left(\sqrt{\frac{\log n}{n h^{d+4}}}\right)$ is the stochastic variation. Recall that in the proof of Theorem $8, Z_{d}$ is

$$
Z_{d}=\sup _{x \in \mathbb{K}}\left\|\nabla \nabla \widehat{p}_{n}(x)-\nabla \nabla p_{h}(x)\right\|_{\max }
$$

By Assumption (K2) and Talagrand's inequality (Giné and Guillou, 2002; Einmahl and Mason, $2005)$, there are constants $c_{3}, c_{4}>0$ such that when $\frac{n h^{d+4}}{\log n} \rightarrow \infty$

$$
P\left(Z_{d}>t\right)<c_{3} \cdot e^{-c_{4} \cdot n h^{d+4} \cdot t^{2}} .
$$

Thus, using equations (50) and (51), when $h^{2 \wedge m(x)}<\frac{a_{0}}{c_{1}}$,

$$
\begin{aligned}
P\left(T_{\widehat{\alpha}_{n}} \stackrel{T}{\approx} T_{\alpha}\right) & \geq P\left(\max _{s=0, \cdots, d}\left|\delta_{n, h, s}^{(2)}\right|<a_{0}\right) \\
& \geq 1-P\left(Z_{d}>\frac{a_{0}}{c_{2}}\right) \\
& \geq 1-c_{3} \cdot e^{-c_{4} \cdot n h^{d+4} \cdot\left(\frac{a_{0}}{c_{2}}\right)^{2}} \\
& =1-c_{3} \cdot e^{-c_{5} \cdot n h^{d+4}}
\end{aligned}
$$

where $c_{5}=c_{4} \cdot\left(\frac{a_{0}}{c_{2}}\right)^{2}>0$. This proves the desired result.

\section{REFERENCES}

S. Balakrishnan, S. Narayanan, A. Rinaldo, A. Singh, and L. Wasserman. Cluster trees on manifolds. In Advances in Neural Information Processing Systems, 2012.

A. Banyaga and D. Hurtubise. Lectures on Morse homology, volume 29. Springer Science \& Business Media, 2013.

Y. Baryshnikov, P. Bubenik, and M. Kahle. Min-type morse theory for configuration spaces of hard spheres. International Mathematics Research Notices, page rnt012, 2013.

O. Bobrowski, S. Mukherjee, J. E. Taylor, et al. Topological consistency via kernel estimation. Bernoulli, 23(1): 288-328, 2017.

P. Bubenik. Statistical topological data analysis using persistence landscapes. Journal of Machine Learning Research, 16(1):77-102, 2015.

B. Cadre. Kernel estimation of density level sets. Journal of multivariate analysis, 97(4):999-1023, 2006.

B. Cadre, B. Pelletier, and P. Pudlo. Clustering by estimation of density level sets at a fixed probability. 2009. URL https://hal.archives-ouvertes.fr/file/index/docid/397437/filename/tlevel.pdf. 
G. Carlsson. Topology and data. Bulletin of the American Mathematical Society, 46(2):255-308, 2009.

K. Chaudhuri and S. Dasgupta. Rates of convergence for the cluster tree. In Advances in Neural Information Processing Systems, pages 343-351, 2010.

K. Chaudhuri, S. Dasgupta, S. Kpotufe, and U. von Luxburg. Consistent procedures for cluster tree estimation and pruning. IEEE Transactions on Information Theory, 60(12):7900-7912, 2014.

F. Chazal, B. T. Fasy, F. Lecci, B. Michel, A. Rinaldo, and L. Wasserman. Robust topological inference: Distance to a measure and kernel distance. arXiv preprint arXiv:1412.7197, 2014.

Y.-C. Chen. Supplementary proofs: Generalized cluster trees and singular measures. doi: COMPLETED BY THE TYPESETTER, 2016.

Y.-C. Chen. A tutorial on kernel density estimation and recent advances. arXiv preprint arXiv:1704.03924, 2017.

Y.-C. Chen and A. Dobra. Measuring human activity spaces with density ranking based on gps data. arXiv preprint arXiv:1708.05017, 2017.

Y.-C. Chen, C. R. Genovese, and L. Wasserman. Density level sets: Asymptotics, inference, and visualization. arXiv:1504.05438, 2015a.

Y.-C. Chen, C. R. Genovese, L. Wasserman, et al. Asymptotic theory for density ridges. The Annals of Statistics, 43 (5):1896-1928, 2015b.

Y.-C. Chen, C. R. Genovese, L. Wasserman, et al. A comprehensive approach to mode clustering. Electronic Journal of Statistics, 10(1):210-241, 2016a.

Y.-C. Chen, J. Kim, S. Balakrishnan, A. Rinaldo, and L. Wasserman. Statistical inference for cluster trees. arXiv preprint arXiv:1605.06416, 2016b.

D. Cohen-Steiner, H. Edelsbrunner, and J. Harer. Stability of persistence diagrams. Discrete ES Computational Geometry, 37(1):103-120, 2007.

H. Edelsbrunner and J. Harer. Persistent homology-a survey. Contemporary mathematics, 453:257-282, 2008.

H. Edelsbrunner and D. Morozov. Persistent homology: theory and practice. In Proceedings of the European Congress of Mathematics, pages 31-50, 2012.

U. Einmahl and D. M. Mason. Uniform in bandwidth consistency of kernel-type function estimators. The Annals of Statistics, 33(3):1380-1403, 2005.

J. Eldridge, M. Belkin, and Y. Wang. Beyond hartigan consistency: Merge distortion metric for hierarchical clustering. In Proceedings of The 28th Conference on Learning Theory, pages 588-606, 2015.

B. T. Fasy, F. Lecci, A. Rinaldo, L. Wasserman, S. Balakrishnan, A. Singh, et al. Confidence sets for persistence diagrams. The Annals of Statistics, 42(6):2301-2339, 2014.

H. Federer. Curvature measures. Trans. Am. Math. Soc, 93, 1959.

G. Friedman. Singular intersection homology. Preprint, 2014.

C. R. Genovese, M. Perone-Pacifico, I. Verdinelli, L. Wasserman, et al. On the path density of a gradient field. The Annals of Statistics, 37(6A):3236-3271, 2009.

C. R. Genovese, M. Perone-Pacifico, I. Verdinelli, and L. Wasserman. Nonparametric ridge estimation. The Annals of Statistics, 42(4):1511-1545, 2014.

E. Giné and A. Guillou. Rates of strong uniform consistency for multivariate kernel density estimators. In Annales de l'Institut Henri Poincare (B) Probability and Statistics, volume 38, pages 907-921. Elsevier, 2002.

M. Goresky and R. MacPherson. Intersection homology theory. Topology, 19(2):135-162, 1980.

M. Goresky and R. MacPherson. Stratified morse theory, volume 14. Springer Science \& Business Media, 2012.

J. A. Hartigan. Consistency of single linkage for high-density clusters. Journal of the American Statistical Association, 1981.

B. P. Kent. Level Set Trees for Applied Statistics. PhD thesis, Carnegie Mellon University, 2013.

J. Klemelä. Visualization of multivariate density estimates with level set trees. Journal of Computational and Graphical Statistics, 13(3), 2004.

J. Klemelä. Visualization of multivariate density estimates with shape trees. Journal of Computational and Graphical Statistics, 15(2):372-397, 2006.

J. Klemelä. Smoothing of multivariate data: density estimation and visualization, volume 737. John Wiley \& Sons, 2009 .

S. Kpotufe and U. V. Luxburg. Pruning nearest neighbor cluster trees. In Proceedings of the 28th International Conference on Machine Learning (ICML-11), pages 225-232, 2011.

T. Laloe and R. Servien. Nonparametric estimation of regression level sets. Journal of the Korean Statistical Society, 2013.

J. Lee. Introduction to Smooth Manifolds, volume 218. Springer Science \& Business Media, 2012. 
E. Mammen and W. Polonik. Confidence regions for level sets. Journal of Multivariate Analysis, 122:202-214, 2013.

D. M. Mason and W. Polonik. Asymptotic normality of plug-in level set estimates. The Annals of Applied Probability, 19(3):1108-1142, 2009.

P. Mattila. Geometry of sets and measures in Euclidean spaces: fractals and rectifiability, volume 44 . Cambridge university press, 1999.

J. W. Milnor. Morse theory. Number 51. Princeton university press, 1963.

I. Molchanov. Empirical estimation of distribution quantiles of random closed sets. Theory of Probability 8 Its Applications, 35(3):594-600, 1991.

M. Morse. Relations between the critical points of a real function of $\mathrm{n}$ independent variables. Transactions of the American Mathematical Society, 27(3):345-396, 1925.

M. Morse. The foundations of a theory of the calculus of variations in the large in $\mathrm{m}$-space (second paper). Transactions of the American Mathematical Society, 32(4):599-631, 1930.

W. Polonik. Measuring mass concentrations and estimating density contour clusters-an excess mass approach. The Annals of Statistics, pages 855-881, 1995.

D. Preiss. Geometry of measures in $\mathbb{R}^{n}$ : distribution, rectifiability, and densities. Annals of Mathematics, 125(3): 537-643, 1987.

A. Rinaldo and L. Wasserman. Generalized density clustering. The Annals of Statistics, 38(5):2678-2722, Oct. 2010. ISSN 0090-5364. . URL http://arxiv.org/abs/0907.3454.

A. Rinaldo, A. Singh, R. Nugent, and L. Wasserman. Stability of density-based clustering. The Journal of Machine Learning Research, 13(1):905-948, 2012.

D. W. Scott. Multivariate density estimation: theory, practice, and visualization. John Wiley \& Sons, 2015.

A. Singh, C. Scott, and R. Nowak. Adaptive hausdorff estimation of density level sets. The Annals of Statistics, 37 (5B):2760-2782, 2009.

I. Steinwart. Adaptive density level set clustering. In COLT, pages 703-738, 2011.

W. Stuetzle. Estimating the cluster tree of a density by analyzing the minimal spanning tree of a sample. $J$. Classification, 20(1):025-047, 2003.

A. B. Tsybakov. On nonparametric estimation of density level sets. The Annals of Statistics, 25(3):948-969, 1997.

L. W. Tu. An introduction to manifolds. Springer Science \& Business Media, 2010.

G. Walther. Granulometric smoothing. The Annals of Statistics, 25(6):2273-2299, 1997.

L. Wasserman. All of nonparametric statistics. Springer Science \& Business Media, 2006.

L. Wasserman. Topological data analysis. arXiv preprint arXiv:1609.08227, 2016.

Department of Statistics

UniVERSiTY OF WASHINGTON

Box 354322

Seattle, WA 98195

E-MAIL: yenchic@uw.edu 